Supporting Information for

\title{
Synthesis of Novel Potent Archazolids: Pharmacology of an Emerging Class of ANTICANCER DRUGS
}

Stephan Scheeff, ${ }^{1}$ Solenne Rivière, ${ }^{1}$ Johal Ruiz, ${ }^{1}$ Aliaa Abdelrahman, ${ }^{2}$ Anna-Christina Schulz-Fincke, ${ }^{2}$ Meryem Köse, ${ }^{2}$ Felix Tiburcy, ${ }^{3}$ Helmut Wieczorek, ${ }^{3}$, Michael Gütschow, ${ }^{2}$ Christa E. Müller, ${ }^{2}$ and Dirk Menche ${ }^{1}$

${ }^{1}$ Kekulé-Institut für Organische Chemie und Biochemie, Universität Bonn Gerhard-Domagk-Str. 1, D-53121 Bonn, Germany

2 Pharmazeutisches Institut, Universität Bonn, An der Immenburg 4, D-53121 Bonn, Germany

${ }^{3}$ Universität Osnabrück, Fachbereich Biologie/Chemie, D-49069 Osnabrück,Germany. 


\section{Contents}

1 Additional Schemes and Material S3

1.1 Structure Activity Relationship . . . . . . . . . . . . . . . . . . . . . . . . . . S3

1.2 Profiling of archazolid A . . . . . . . . . . . . . . . . . . . . . . . S6

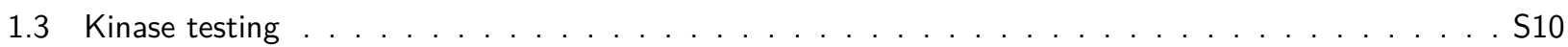

1.4 MTT Assay . . . . . . . . . . . . . . . . . . . . . . . . . . . . . . . . . . . . . . . . . . S13

$1.5 \quad \mathrm{~A}_{3}$ Adenosine Receptor Radioligand Binding Assay . . . . . . . . . . . . . . . . . . . . . . . . . . . . . . S14

1.6 Protease Assays . . . . . . . . . . . . . . . . . . . . . . . . . . . . . . . . . . . . . . . . . . . . . . S14

1.7 CYP Inhibition . . . . . . . . . . . . . . . . . . . . . . . . . . . . . . . . . . . . . . . . S14

1.8 Inhibition by $\mathrm{P} 2 \mathrm{Y}$ and $\mathrm{P} 2 \mathrm{X}$ receptors and radioligand binding studies for human $\mathrm{P} 2 \mathrm{X} 3$ receptors . . . S15

1.9 Metabolic Stability . . . . . . . . . . . . . . . . . . . . . . . . . . . . . S16

1.10 In-vitro studies to determine physicochemical and pharmacokinetic properties . . . . . . . . . . . S17

1.11 Water solubility . . . . . . . . . . . . . . . . . . . . . . . . . . S17

2 Additional Information Chemical Synthesis $\quad \mathbf{S 1 9}$

2.1 NMR assignment . . . . . . . . . . . . . . . . . . . . . . . . . . . . . . . . . . . . . . . . . . . . .

2.1.1 Assignment for iso Archazolids $\mathrm{A}_{1}(4)$ and $\mathrm{A}_{2}(5) \ldots \ldots \ldots$. . . . . . . . . . . . . S21

2.1.2 Assignment for Aldehyde 9 . . . . . . . . . . . . . . . . . . . . . . . . . . . . . . . S22

2.1 .3 Assignment for Ketone 12 . . . . . . . . . . . . . . . . . . . . . . . . . . . . . . . . . . . S22

2.1 .4 Assignment for Alcohol 13 . . . . . . . . . . . . . . . . . . . . . . . . . . . . . . . . S23

2.1 .5 Assignment for Desmethylarchazolid F (6) . . . . . . . . . . . . . . . . . . . . . . . . . . S25

2.1 .6 Assignment for Ketone 18 . . . . . . . . . . . . . . . . . . . . . . . . . . . . . . . S26

2.1 .7 Assignment for Alcohol $22 \ldots \ldots \ldots \ldots$. . . . . . . . . . . . . . . . . . . . . . . . . . . S27

2.1 .8 Assignment for Methyl Ether 19 . . . . . . . . . . . . . . . . . . . . . . . . . . . . . . . . . . S27

2.1 .9 Assignment for Alcohol 23 . . . . . . . . . . . . . . . . . . . . . . . . . . . . . . . . . . . . . S28

2.1.10 Assignment for Ester 20 . . . . . . . . . . . . . . . . . . . . . . . . . . . . . . . . . . . . . . . . . . . . . . S29

2.1.11 Assignment for Archazolog (7) . . . . . . . . . . . . . . . . . . . . . . . . . . . . S30

2.2 HPLC configuration for semipreparative separation . . . . . . . . . . . . . . . . . . . . S31

2.2 .1 Isomerization of Archazolid B . . . . . . . . . . . . . . . . . . . . . . . S32

2.2 .2 Isomerization of Archazolid A . . . . . . . . . . . . . . . . . . . . . . . . . . . . . . S34

2.2 .3 Synthesis of desmethyl-archazolid F . . . . . . . . . . . . . . . . . . . . . . . S36

2.2 .4 Synthesis of archazolog . . . . . . . . . . . . . . . . . . . . . . . . . . . S38

2.3 Proof of purity . . . . . . . . . . . . . . . . . . . . . . . . . . . . . . . . . . . . .

3 Copies of NMR spectra $\quad 563$ 


\section{Additional Schemes and Material}

\subsection{Structure Activity Relationship}

For a better understanding the biological data of naturally ${ }^{a}$ and of derivatives of archazolids ${ }^{b}$ were compared.

\footnotetext{
${ }^{a}$ N. Horstmann, S. Essig, S. Bockelmann, H. Wieczorek, M. Huss, F. Sasse, D. Menche, J. Nat. Prod. 2011, 74, 1100; S. Dreisigacker D. Latek, S. Bockelmann, M. Huss, H. Wieczorek, S. Filipek, H. Gohlke, D. Menche, T. Carlomagno, J. Chem. Inf. Model. 2012, 52, 2265; D. Menche, J., H. Steinmetz, M. Huss, H. Wieczorek, F. Sasse, J. Antibiot. 2007, 60, 328; D. Menche, J., H. Steinmetz, M. Huss, H. Wieczorek, F. Sasse, Eur. J. Org. Chem. 2007, 1196.

bS. Dreisigacker, D. Latek, S. Bockelmann, M. Huss, H. Wieczorek, S. Filipek, H. Gohlke, D. Menche, T. Carlomagno, J. Chem. Inf Model. 2012, 52, 2265; E. Persch, T. Basile, S. Bockelmann, M. Huss, H. Wieczorek, T. Carlomagno, D. Menche, Bioorg. Med. Chem. Lett. 2012, 22, 7735; D. Menche, J. Hassfeld, F. Sasse, M. Huss, H. Wieczorek, Bioorg. Med. Chem. Lett. 2007, $17,1732$.
} 


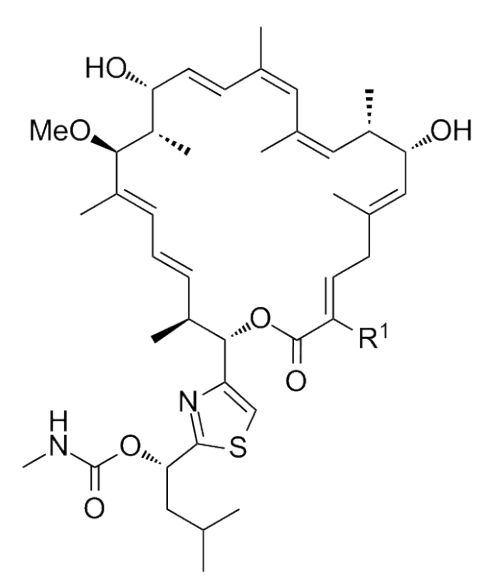

Archazolid $A\left(R^{1}=\mathrm{Me}\right)$ :

$I_{50}$ (V-ATPase): $0.6 \mathrm{nM}, \mathrm{IC}_{50}$ (L-929): $0.81 \mathrm{nM}$ Archazolid B $\left(\mathrm{R}^{1}=\mathrm{H}\right)$ :

$I C_{50}$ (V-ATPase): $0.6 \mathrm{nM}, \mathrm{IC}_{50}$ (L-929): $1.1 \mathrm{nM}$

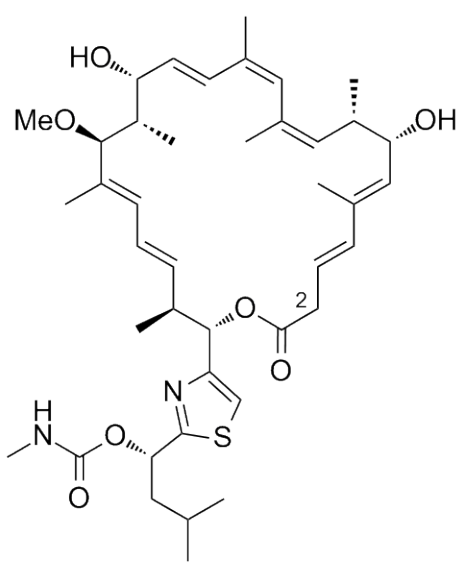

Archazolid F:

$I_{50}$ (V-ATPase): $0.7 \mathrm{nM}, \mathrm{IC}_{50}$ (L-929): $0.11 \mathrm{nM}$

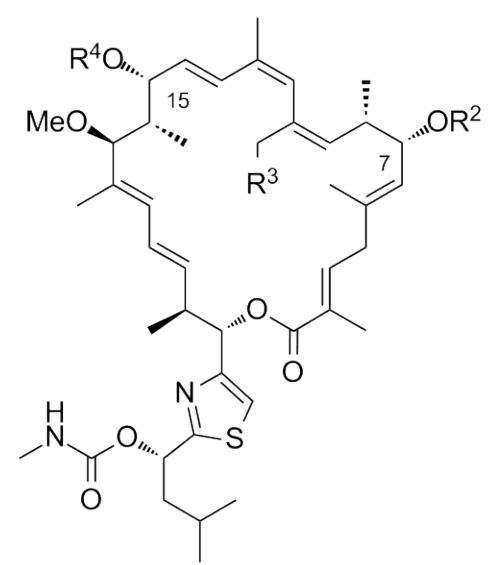

Archazolid C ( $\mathrm{R}^{2}=\beta$-D-Glc, $\left.\mathrm{R}^{3}=\mathrm{H}, \mathrm{R}^{4}=\mathrm{H}\right)$ : $I_{50}$ (V-ATPase): $210 \mathrm{nM}, \mathrm{IC}_{50}$ (L-929): $1600 \mathrm{nM}$ Archazolid D ( $\mathrm{R}^{2}=\beta$-D-Glc, $\left.\mathrm{R}^{3}=\mathrm{OH}, \mathrm{R}^{4}=\mathrm{H}\right)$ : $I_{50}$ (V-ATPase): $1200 \mathrm{nM}, \mathrm{IC}_{50}$ (L-929): $330 \mathrm{nM}$ Archazolid E $\left(\mathrm{R}^{2}=\mathrm{H}, \mathrm{R}^{3}=\mathrm{H}, \mathrm{R}^{4}=\beta-\mathrm{D}-\mathrm{Gl}\right)$ : ${ }^{\prime} C_{50}$ (L-929): $510 \mathrm{nM}$

Figure S1. Biological profile of naturally occurring archazolids. 


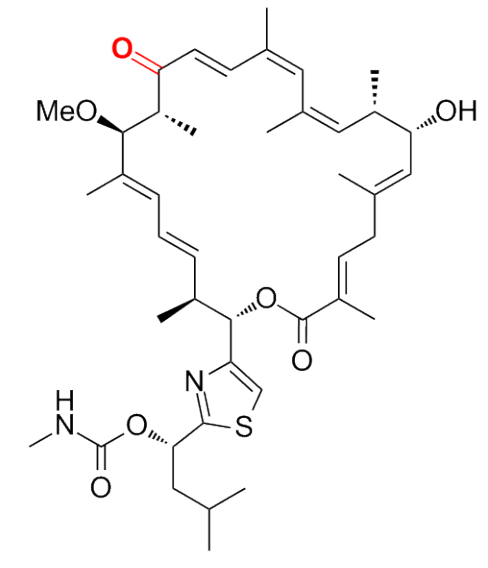

15-Dehydroarchazolid A

$\mathrm{IC}_{50}$ (V-ATPase): $13 \mathrm{nM}, \mathrm{IC}_{50}$ (L-929): >5000 nM

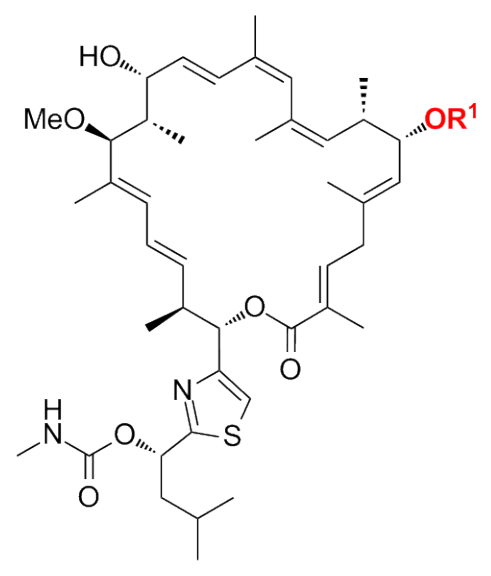

7-OTBS-Archazolid A ( ${ }^{1}=$ TBS):

$I_{50}$ (V-ATPase): $140 \mathrm{nM}, \mathrm{IC}_{50}$ (L-929): $940 \mathrm{nM}$ 7-Op-NBZ-Archazolid A $\left(R^{1}=p-N B z\right):$

$\mathrm{IC}_{50}$ (V-ATPase): $250 \mathrm{nM}$; IC 50 (L-929): $290 \mathrm{nM}$

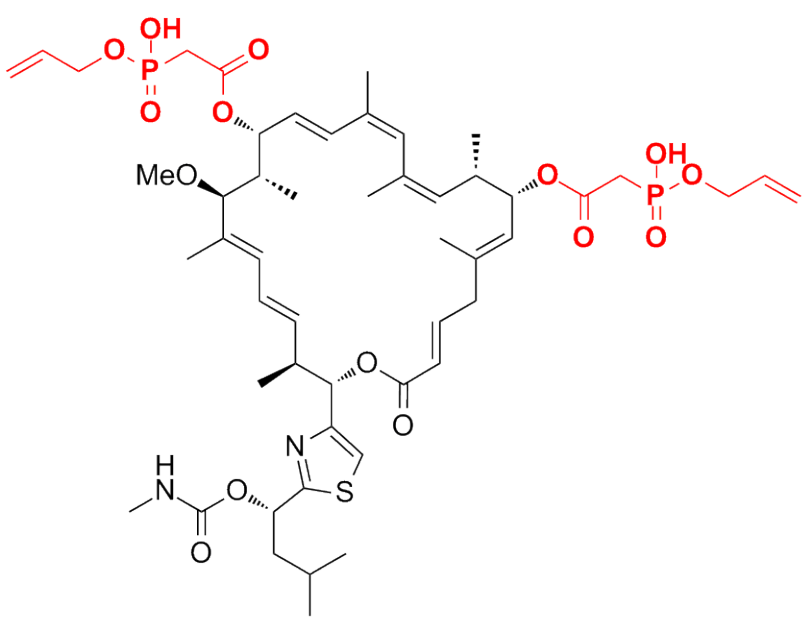

Phosphonate-derivative of archazolid $B$ $\mathrm{IC}_{50}$ (V-ATPase): >10000 nM

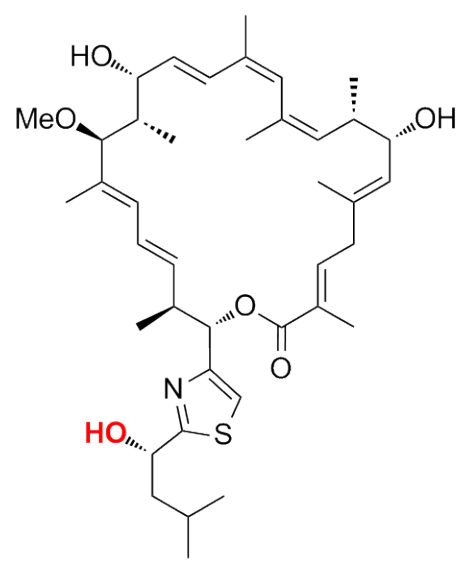

Descarbamoyl-archazolid A

$\mathrm{IC}_{50}$ (V-ATPase): $5 \mathrm{nM}, \mathrm{IC}_{50}$ (L-929): $14 \mathrm{nM}$

Figure S2. Archazolid derivatives and their biological data. 


\subsection{Profiling of archazolid A}

Table S1: Profiling of archazolid A.

\begin{tabular}{|c|c|c|c|c|c|c|c|c|c|}
\hline & $\begin{array}{l}\text { Test Con- } \\
\text { centration } \\
{[\mathrm{M}]}\end{array}$ & $\begin{array}{l}\% \text { Inhi- } \\
\text { bition } \\
\text { of } \\
\text { contr. } \\
\text { spec. } \\
\text { binding }\end{array}$ & $\begin{array}{l}\% \text { of } \\
\text { cific } B\end{array}$ & $\begin{array}{l}\text { Control } \\
\text { ding }\end{array}$ & Mean & $\begin{array}{l}\text { Reference } \\
\text { Compound }\end{array}$ & $\begin{array}{l}\mathrm{IC}_{50} \\
\operatorname{Ref} \\
(\mathrm{M})\end{array}$ & $\begin{array}{l}\mathrm{K}_{i} \\
\operatorname{Ref} \\
(\mathrm{M})\end{array}$ & $\begin{array}{l}\mathrm{nH} \\
\mathrm{Ref}\end{array}$ \\
\hline $\begin{array}{l}\text { A1 (h) (antagonist ra- } \\
\text { dioligand) }\end{array}$ & $1 \mathrm{E}-05$ & 7 & 94.1 & 92.3 & 93.2 & DPCPX & $\begin{array}{l}1.4 \mathrm{E}- \\
09\end{array}$ & $\begin{array}{l}9.1 \mathrm{E}- \\
10\end{array}$ & 1.7 \\
\hline $\begin{array}{l}\text { A2A }(\mathrm{h}) \text { (agonist radi- } \\
\text { oligand) }\end{array}$ & $1 \mathrm{E}-05$ & 1 & 96.5 & 101.3 & 98.9 & NECA & $\begin{array}{l}2.7 \mathrm{E}- \\
08\end{array}$ & $\begin{array}{l}2.2 \mathrm{E}- \\
08\end{array}$ & 1.3 \\
\hline $\begin{array}{l}\text { A3 }(\mathrm{h}) \text { (agonist radioli- } \\
\text { gand) }\end{array}$ & $1 \mathrm{E}-05$ & 85 & 16.0 & 14.0 & 15 & IB-MECA & $\begin{array}{l}3.2 \mathrm{E}- \\
10\end{array}$ & $\begin{array}{l}1.9 \mathrm{E}- \\
10\end{array}$ & 0.8 \\
\hline $\begin{array}{l}\text { alpha } 1 \text { (non-selective) } \\
\text { (antagonist radioli- } \\
\text { gand) }\end{array}$ & $1 \mathrm{E}-05$ & 2 & 98.8 & 97.4 & 98.1 & prazosin & $3 E-10$ & $\begin{array}{l}7.9 \mathrm{E}- \\
11\end{array}$ & 0.9 \\
\hline $\begin{array}{l}\text { alpha } 2 \text { (non-selective) } \\
\text { (antagonist radioli- } \\
\text { gand) }\end{array}$ & $1 \mathrm{E}-05$ & -7 & 100.7 & 112.8 & 106.7 & yohimbine & $\begin{array}{l}5.4 \mathrm{E}- \\
08\end{array}$ & $\begin{array}{l}2.3 \mathrm{E}- \\
08\end{array}$ & 0.6 \\
\hline $\begin{array}{l}\text { beta } 1(\mathrm{~h}) \text { (agonist ra- } \\
\text { dioligand) }\end{array}$ & $1 \mathrm{E}-05$ & 3 & 103.7 & 90.3 & 97 & atenolol & $\begin{array}{l}3.3 \mathrm{E}- \\
07\end{array}$ & $\begin{array}{l}1.9 \mathrm{E}- \\
07\end{array}$ & 1.1 \\
\hline $\begin{array}{l}\text { beta } 2(\mathrm{~h}) \text { (agonist ra- } \\
\text { dioligand) }\end{array}$ & $1 \mathrm{E}-05$ & -2 & 97.3 & 106.7 & 102 & ICI 118551 & $\begin{array}{l}9.1 \mathrm{E}- \\
10\end{array}$ & $3 E-10$ & 1.3 \\
\hline $\begin{array}{l}\text { AT1 (h) (antagonist } \\
\text { radioligand) }\end{array}$ & $1 \mathrm{E}-05$ & 11 & 85.2 & 93.3 & 89.3 & saralasin & $\begin{array}{l}9.3 \mathrm{E}- \\
10\end{array}$ & $\begin{array}{l}4.6 \mathrm{E}- \\
10\end{array}$ & 1.0 \\
\hline $\begin{array}{l}\text { AT2 }(h) \text { (agonist radi- } \\
\text { oligand) }\end{array}$ & $1 \mathrm{E}-05$ & 8 & 93.8 & 89.5 & 91.7 & angiotensin-II & $\begin{array}{l}1.6 \mathrm{E}- \\
10\end{array}$ & $\begin{array}{l}7.9 \mathrm{E}- \\
11\end{array}$ & 1.1 \\
\hline $\begin{array}{l}\text { BZD (central) (ago- } \\
\text { nist radioligand) }\end{array}$ & $1 \mathrm{E}-05$ & -25 & 140.1 & 110.6 & 125.4 & diazepam & $\begin{array}{l}9.8 \mathrm{E}- \\
09\end{array}$ & $\begin{array}{l}8.3 \mathrm{E}- \\
09\end{array}$ & 0.8 \\
\hline $\begin{array}{l}\text { BZD (peripheral) (an- } \\
\text { tagonist radioligand) }\end{array}$ & $1 \mathrm{E}-05$ & 34 & 62.0 & 70.2 & 66.1 & PK 11195 & $\begin{array}{l}2.2 \mathrm{E}- \\
09\end{array}$ & $2 \mathrm{E}-09$ & 0.8 \\
\hline $\begin{array}{l}\text { BB (non-selective) } \\
\text { (agonist radioligand) }\end{array}$ & $1 \mathrm{E}-05$ & 5 & 102.0 & 87.8 & 94.9 & bombesin & $\begin{array}{l}3.4 \mathrm{E}- \\
10\end{array}$ & $\begin{array}{l}3.4 \mathrm{E}- \\
10\end{array}$ & 0.9 \\
\hline $\begin{array}{l}\text { B2 }(\mathrm{h}) \text { (agonist radioli- } \\
\text { gand) }\end{array}$ & $1 \mathrm{E}-05$ & -1 & 107.1 & 95.2 & 101.1 & NPC 567 & $\begin{array}{l}2.4 \mathrm{E}- \\
08\end{array}$ & $\begin{array}{l}1.2 \mathrm{E}- \\
08\end{array}$ & 1.0 \\
\hline $\begin{array}{l}\text { CGRP }(h) \text { (agonist ra- } \\
\text { dioligand) }\end{array}$ & $1 \mathrm{E}-05$ & -36 & 130.4 & 142.2 & 136.3 & hCGRPalpha & $\begin{array}{l}3.2 \mathrm{E}- \\
11\end{array}$ & $\begin{array}{l}2.1 \mathrm{E}- \\
11\end{array}$ & 0.7 \\
\hline $\begin{array}{l}\text { CB1 }(h) \text { (agonist radi- } \\
\text { oligand) }\end{array}$ & $1 \mathrm{E}-05$ & 1 & 99.5 & 97.9 & 98.7 & CP 55940 & $\begin{array}{l}1.2 \mathrm{E}- \\
09\end{array}$ & $1 \mathrm{E}-09$ & 0.7 \\
\hline $\begin{array}{l}\text { CCK1 }(\mathrm{CCKA})(\mathrm{h})(\mathrm{ag}- \\
\text { onist radioligand) }\end{array}$ & $1 \mathrm{E}-05$ & -13 & 106.8 & 118.3 & 112.5 & CCK-8s & $\begin{array}{l}3.4 \mathrm{E}- \\
10\end{array}$ & $\begin{array}{l}2.5 \mathrm{E}- \\
10\end{array}$ & 0.9 \\
\hline $\begin{array}{l}\text { CCK2 (CCKB) }(\mathrm{h})(\mathrm{ag}- \\
\text { onist radioligand) }\end{array}$ & $1 \mathrm{E}-05$ & -36 & 136.5 & 135.6 & 136 & CCK-8s & $\begin{array}{l}1.3 \mathrm{E}- \\
10\end{array}$ & $\begin{array}{l}5.4 \mathrm{E}- \\
11\end{array}$ & 1.0 \\
\hline
\end{tabular}


Table S1: Profiling of archazolid A.

\begin{tabular}{|c|c|c|c|c|c|c|c|c|c|}
\hline & $\begin{array}{l}\text { Test Con- } \\
\text { centration } \\
{[\mathrm{M}]}\end{array}$ & $\begin{array}{l}\% \text { Inhi- } \\
\text { bition } \\
\text { of } \\
\text { contr. } \\
\text { spec. } \\
\text { binding }\end{array}$ & $\begin{array}{l}\% \text { of } \\
\text { cific } B\end{array}$ & $\begin{array}{l}\text { Control } \\
\text { ding }\end{array}$ & pe- & $\begin{array}{l}\text { Reference } \\
\text { Compound }\end{array}$ & $\begin{array}{l}\mathrm{IC}_{50} \\
\operatorname{Ref} \\
(\mathrm{M})\end{array}$ & $\begin{array}{l}\mathrm{K}_{i} \\
\mathrm{Ref} \\
(\mathrm{M})\end{array}$ & $\begin{array}{l}\mathrm{nH} \\
\text { Ref }\end{array}$ \\
\hline $\begin{array}{l}\text { D1 (h) (antagonist ra- } \\
\text { dioligand) }\end{array}$ & $1 \mathrm{E}-05$ & 12 & 92.0 & 84.2 & 88.1 & SCH 23390 & $\begin{array}{l}1.5 \mathrm{E}- \\
10\end{array}$ & $\begin{array}{l}6.1 \mathrm{E}- \\
11\end{array}$ & 0.7 \\
\hline $\begin{array}{l}\mathrm{D} 2 \mathrm{~S}(\mathrm{~h}) \text { (antagonist } \\
\text { radioligand) }\end{array}$ & $1 \mathrm{E}-05$ & -4 & 109.6 & 97.6 & 103.6 & $(+)$ butaclamol & $\begin{array}{l}1.3 \mathrm{E}- \\
09\end{array}$ & $\begin{array}{l}4.4 \mathrm{E}- \\
10\end{array}$ & 0.9 \\
\hline $\begin{array}{l}\text { D3 (h) (antagonist ra- } \\
\text { dioligand) }\end{array}$ & $1 \mathrm{E}-05$ & 3 & 108.7 & 85.6 & 97.1 & $(+)$ butaclamol & $\begin{array}{l}1.5 \mathrm{E}- \\
09\end{array}$ & $\begin{array}{l}3.4 \mathrm{E}- \\
10\end{array}$ & 1.0 \\
\hline $\begin{array}{l}\text { D4.4 (h) (antagonist } \\
\text { radioligand) }\end{array}$ & $1 \mathrm{E}-05$ & -4 & 111.9 & 96.4 & 104.1 & clozapine & $\begin{array}{l}5.8 \mathrm{E}- \\
08\end{array}$ & $\begin{array}{l}2.2 \mathrm{E}- \\
08\end{array}$ & 0.8 \\
\hline $\begin{array}{l}\text { D5 (h) (antagonist ra- } \\
\text { dioligand) }\end{array}$ & $1 \mathrm{E}-05$ & 11 & 85.1 & 92.4 & 88.8 & SCH 23390 & $\begin{array}{l}6.1 \mathrm{E}- \\
10\end{array}$ & $\begin{array}{l}2.8 \mathrm{E}- \\
10\end{array}$ & 1.1 \\
\hline $\begin{array}{l}\text { ETA }(h) \text { (agonist radi- } \\
\text { oligand) }\end{array}$ & $1 \mathrm{E}-05$ & 2 & 101.0 & 94.5 & 97.7 & endothelin-1 & $\begin{array}{l}4.2 \mathrm{E}- \\
11\end{array}$ & $\begin{array}{l}2.1 \mathrm{E}- \\
11\end{array}$ & 0.9 \\
\hline $\begin{array}{l}\text { ETB }(h) \text { (agonist radi- } \\
\text { oligand) }\end{array}$ & $1 \mathrm{E}-05$ & -3 & 95.9 & 109.2 & 102.5 & endothelin-3 & $\begin{array}{l}2.5 \mathrm{E}- \\
11\end{array}$ & $\begin{array}{l}1.4 \mathrm{E}- \\
11\end{array}$ & 1.1 \\
\hline $\begin{array}{l}\text { GABA (non-selective) } \\
\text { (agonist radioligand) }\end{array}$ & $1 \mathrm{E}-05$ & 9 & 94.3 & 88.5 & 91.4 & GABA & $\begin{array}{l}2.4 \mathrm{E}- \\
08\end{array}$ & $\begin{array}{l}1.4 \mathrm{E}- \\
08\end{array}$ & 0.8 \\
\hline $\begin{array}{l}\text { GAL1 }(\mathrm{h}) \text { (agonist ra- } \\
\text { dioligand) }\end{array}$ & $1 \mathrm{E}-05$ & -3 & 99.8 & 105.7 & 102.7 & galanin & $\begin{array}{l}1.9 \mathrm{E}- \\
10\end{array}$ & $\begin{array}{l}5.4 \mathrm{E}- \\
11\end{array}$ & 1.3 \\
\hline $\begin{array}{l}\text { GAL2 (h) (agonist ra- } \\
\text { dioligand) }\end{array}$ & $1 \mathrm{E}-05$ & -4 & 107.2 & 101.0 & 104.1 & galanin & $\begin{array}{l}5.9 \mathrm{E}- \\
10\end{array}$ & $\begin{array}{l}5.5 \mathrm{E}- \\
10\end{array}$ & 1.7 \\
\hline $\begin{array}{l}\text { CXCR2 (IL-8B) (h) } \\
\text { (agonist radioligand) }\end{array}$ & $1 \mathrm{E}-05$ & 17 & 82.4 & 83.7 & 83 & IL-8 & $\begin{array}{l}1.2 \mathrm{E}- \\
10\end{array}$ & $\begin{array}{l}5.8 \mathrm{E}- \\
11\end{array}$ & 1.4 \\
\hline $\begin{array}{l}\text { CCR1 (h) (agonist ra- } \\
\text { dioligand) }\end{array}$ & $1 \mathrm{E}-05$ & -3 & 103.3 & 103.7 & 103.5 & MIP-1alpha & $\begin{array}{l}6.3 \mathrm{E}- \\
11\end{array}$ & $\begin{array}{l}4.2 \mathrm{E}- \\
11\end{array}$ & 2.6 \\
\hline $\begin{array}{l}\text { TNF-alpha (h) (ago- } \\
\text { nist radioligand) }\end{array}$ & $1 \mathrm{E}-05$ & -53 & 168.4 & 137.9 & 153.2 & TNF-alpha & $\begin{array}{l}5.9 \mathrm{E}- \\
11\end{array}$ & $2 \mathrm{E}-11$ & 1.3 \\
\hline $\begin{array}{l}\mathrm{H} 1 \text { (h) (antagonist ra- } \\
\text { dioligand) }\end{array}$ & $1 \mathrm{E}-05$ & 16 & 82.8 & 85.3 & 84.1 & pyrilamine & $\begin{array}{l}1.8 \mathrm{E}- \\
09\end{array}$ & $\begin{array}{l}1.1 \mathrm{E}- \\
09\end{array}$ & 1.0 \\
\hline $\begin{array}{l}\mathrm{H} 2 \text { (h) (antagonist ra- } \\
\text { dioligand) }\end{array}$ & $1 \mathrm{E}-05$ & -40 & 150.6 & 130.0 & 140.3 & cimetidine & $\begin{array}{l}5.6 \mathrm{E}- \\
07\end{array}$ & $\begin{array}{l}5.5 \mathrm{E}- \\
07\end{array}$ & 0.8 \\
\hline $\begin{array}{l}\text { MC4 }(h) \text { (agonist radi- } \\
\text { oligand) }\end{array}$ & $1 \mathrm{E}-05$ & 11 & 88.6 & 89.5 & 89 & $\begin{array}{l}\text { NDP-alpha } \\
-\mathrm{MSH}\end{array}$ & $\begin{array}{l}4.5 \mathrm{E}- \\
10\end{array}$ & $\begin{array}{l}4.2 \mathrm{E}- \\
10\end{array}$ & 0.8 \\
\hline $\begin{array}{l}\text { MT1 }(\mathrm{ML1A})(\mathrm{h})(\mathrm{ag}- \\
\text { onist radioligand) }\end{array}$ & $1 \mathrm{E}-05$ & 35 & 71.0 & 59.8 & 65.4 & melatonin & $6 \mathrm{E}-10$ & $\begin{array}{l}4.8 \mathrm{E}- \\
10\end{array}$ & 1.0 \\
\hline $\begin{array}{l}\text { M1 (h) (antagonist ra- } \\
\text { dioligand) }\end{array}$ & $1 \mathrm{E}-05$ & 19 & 82.8 & 78.8 & 80.8 & pirenzepine & $\begin{array}{l}2.1 \mathrm{E}- \\
08\end{array}$ & $\begin{array}{l}1.8 \mathrm{E}- \\
08\end{array}$ & 1.2 \\
\hline $\begin{array}{l}\text { M2 (h) (antagonist ra- } \\
\text { dioligand) }\end{array}$ & $1 \mathrm{E}-05$ & 40 & 64.3 & 55.7 & 60 & methoctramine & $\begin{array}{l}3.8 \mathrm{E}- \\
08\end{array}$ & $\begin{array}{l}2.7 \mathrm{E}- \\
08\end{array}$ & 0.9 \\
\hline
\end{tabular}


Table S1: Profiling of archazolid A.

\begin{tabular}{|c|c|c|c|c|c|c|c|c|c|}
\hline & $\begin{array}{l}\text { Test Con- } \\
\text { centration } \\
{[\mathrm{M}]}\end{array}$ & $\begin{array}{l}\% \text { Inhi- } \\
\text { bition } \\
\text { of } \\
\text { contr. } \\
\text { spec. } \\
\text { binding }\end{array}$ & $\begin{array}{l}\% \text { of } \\
\text { cific } B\end{array}$ & $\begin{array}{l}\text { Control } \\
\text { ding }\end{array}$ & pe- & $\begin{array}{l}\text { Reference } \\
\text { Compound }\end{array}$ & $\begin{array}{l}\mathrm{IC}_{50} \\
\operatorname{Ref} \\
(\mathrm{M})\end{array}$ & $\begin{array}{l}\mathrm{K}_{i} \\
\mathrm{Ref} \\
(\mathrm{M})\end{array}$ & $\begin{array}{l}\mathrm{nH} \\
\text { Ref }\end{array}$ \\
\hline $\begin{array}{l}\text { M3 (h) (antagonist ra- } \\
\text { dioligand) }\end{array}$ & $1 \mathrm{E}-05$ & 11 & 86.2 & 92.4 & 89.3 & 4-DAMP & $\begin{array}{l}1.2 \mathrm{E}- \\
09\end{array}$ & $\begin{array}{l}8.6 \mathrm{E}- \\
10\end{array}$ & 2.0 \\
\hline $\begin{array}{l}\text { M4 (h) (antagonist ra- } \\
\text { dioligand) }\end{array}$ & $1 \mathrm{E}-05$ & 13 & 85.6 & 89.1 & 87.4 & 4-DAMP & $\begin{array}{l}4.6 \mathrm{E}- \\
10\end{array}$ & $\begin{array}{l}2.8 \mathrm{E}- \\
10\end{array}$ & 1.1 \\
\hline $\begin{array}{l}\text { M5 (h) (antagonist ra- } \\
\text { dioligand) }\end{array}$ & $1 \mathrm{E}-05$ & 16 & 86.7 & 80.8 & 83.7 & 4-DAMP & $\begin{array}{l}6.4 \mathrm{E}- \\
10\end{array}$ & $\begin{array}{l}3.2 \mathrm{E}- \\
10\end{array}$ & 1.0 \\
\hline $\begin{array}{l}\text { NK1 (h) (agonist radi- } \\
\text { oligand) }\end{array}$ & $1 \mathrm{E}-05$ & 1 & 100.7 & 97.3 & 99 & $\begin{array}{l}{[\text { Sar9, Met }(\mathrm{O} 2) 11} \\
\text { SP }\end{array}$ & $\begin{array}{l}6.2 \mathrm{E}- \\
10\end{array}$ & $\begin{array}{l}2.7 \mathrm{E}- \\
10\end{array}$ & 0.8 \\
\hline $\begin{array}{l}\text { NK2 (h) (agonist radi- } \\
\text { oligand) }\end{array}$ & $1 \mathrm{E}-05$ & 35 & 66.8 & 62.7 & 64.8 & $\begin{array}{l}\text { [Nleu10]-NKA } \\
(4-10)\end{array}$ & $\begin{array}{l}3.2 \mathrm{E}- \\
09\end{array}$ & $\begin{array}{l}1.7 \mathrm{E}- \\
09\end{array}$ & 0.8 \\
\hline $\begin{array}{l}\text { NK3 (h) (antagonist } \\
\text { radioligand) }\end{array}$ & $1 \mathrm{E}-05$ & -2 & 103.0 & 100.3 & 101.7 & SB 222200 & $6 \mathrm{E}-09$ & $\begin{array}{l}3.3 \mathrm{E}- \\
09\end{array}$ & 0.9 \\
\hline $\begin{array}{l}\text { Y1 (h) (agonist radioli- } \\
\text { gand) }\end{array}$ & $1 \mathrm{E}-05$ & -1 & 103.4 & 98.6 & 101 & NPY & $\begin{array}{l}1.6 \mathrm{E}- \\
10\end{array}$ & $\begin{array}{l}1.1 \mathrm{E}- \\
10\end{array}$ & 1.4 \\
\hline $\begin{array}{l}\text { Y2 }(\mathrm{h}) \text { (agonist radioli- } \\
\text { gand) }\end{array}$ & $1 \mathrm{E}-05$ & -24 & 108.4 & 139.1 & 123.8 & NPY & $\begin{array}{l}7.4 \mathrm{E}- \\
11\end{array}$ & $\begin{array}{l}2.9 \mathrm{E}- \\
11\end{array}$ & 2.8 \\
\hline $\begin{array}{l}\text { NTS1 }(\mathrm{NT} 1)(\mathrm{h}) \text { (ago- } \\
\text { nist radioligand) }\end{array}$ & $1 \mathrm{E}-05$ & -8 & 101.3 & 115.6 & 108.4 & neurotensin & $\begin{array}{l}4.2 \mathrm{E}- \\
10\end{array}$ & $\begin{array}{l}3.5 \mathrm{E}- \\
10\end{array}$ & 0.8 \\
\hline $\begin{array}{l}\text { delta } 2(\mathrm{DOP})(\mathrm{h})(\mathrm{ag}- \\
\text { onist radioligand) }\end{array}$ & $1 \mathrm{E}-05$ & 38 & 68.4 & 55.1 & 61.7 & DPDPE & $\begin{array}{l}1.7 \mathrm{E}- \\
09\end{array}$ & $\begin{array}{l}9.8 \mathrm{E}- \\
10\end{array}$ & 0.8 \\
\hline $\begin{array}{l}\text { kappa (KOP) (agonist } \\
\text { radioligand) }\end{array}$ & $1 \mathrm{E}-05$ & 23 & 78.8 & 75.4 & 77.1 & U 50488 & $\begin{array}{l}9.7 \mathrm{E}- \\
10\end{array}$ & $\begin{array}{l}6.5 \mathrm{E}- \\
10\end{array}$ & 1.3 \\
\hline $\begin{array}{l}\text { mu }(\mathrm{MOP})(\mathrm{h}) \text { (ago- } \\
\text { nist radioligand) }\end{array}$ & $1 \mathrm{E}-05$ & 16 & 85.9 & 81.7 & 83.8 & DAMGO & $\begin{array}{l}9.8 \mathrm{E}- \\
10\end{array}$ & $4 \mathrm{E}-10$ & 1.0 \\
\hline $\begin{array}{l}\text { NOP (ORL1) (h) (ag- } \\
\text { onist radioligand) }\end{array}$ & $1 \mathrm{E}-05$ & 12 & 88.2 & 88.0 & 88.1 & nociceptin & $\begin{array}{l}5.6 \mathrm{E}- \\
10\end{array}$ & $\begin{array}{l}1.1 \mathrm{E}- \\
10\end{array}$ & 1.1 \\
\hline $\begin{array}{l}\text { PAC1 (PACAP) (h) } \\
\text { (agonist radioligand) }\end{array}$ & $1 \mathrm{E}-05$ & -93 & 187.5 & 197.5 & 192.5 & PACAP1-38 & $\begin{array}{l}9.9 \mathrm{E}- \\
11\end{array}$ & $\begin{array}{l}8.5 \mathrm{E}- \\
11\end{array}$ & 0.9 \\
\hline $\begin{array}{l}\text { PPARgamma }(\mathrm{h}) \text { (ag- } \\
\text { onist radioligand) }\end{array}$ & $1 \mathrm{E}-05$ & 82 & 18.1 & 17.5 & 17.8 & rosiglitazone & $\begin{array}{l}1.4 \mathrm{E}- \\
08\end{array}$ & $\begin{array}{l}7.5 \mathrm{E}- \\
09\end{array}$ & 1.0 \\
\hline $\begin{array}{l}\text { PCP (antagonist radi- } \\
\text { oligand) }\end{array}$ & $1 \mathrm{E}-05$ & -4 & 115.4 & 93.3 & 104.4 & MK 801 & $\begin{array}{l}5.5 \mathrm{E}- \\
09\end{array}$ & $\begin{array}{l}3.1 \mathrm{E}- \\
09\end{array}$ & 0.8 \\
\hline $\begin{array}{l}\text { EP2 }(h) \text { (agonist radi- } \\
\text { oligand) }\end{array}$ & $1 \mathrm{E}-05$ & 19 & 81.3 & 80.2 & 80.8 & PGE2 & $\begin{array}{l}1.5 \mathrm{E}- \\
09\end{array}$ & $\begin{array}{l}7.5 \mathrm{E}- \\
10\end{array}$ & 1.9 \\
\hline $\begin{array}{l}\text { EP4 }(h) \text { (agonist radi- } \\
\text { oligand) }\end{array}$ & $1 \mathrm{E}-05$ & 5 & 97.4 & 93.4 & 95.4 & PGE2 & $\begin{array}{l}4.3 \mathrm{E}- \\
10\end{array}$ & $\begin{array}{l}1.6 \mathrm{E}- \\
10\end{array}$ & 1.0 \\
\hline $\begin{array}{l}\mathrm{IP}(\mathrm{PGI})(\mathrm{h}) \text { (agonist } \\
\text { radioligand) }\end{array}$ & $1 \mathrm{E}-05$ & 0 & 101.0 & 98.2 & 99.6 & iloprost & $\begin{array}{l}1.2 \mathrm{E}- \\
08\end{array}$ & $\begin{array}{l}6.6 \mathrm{E}- \\
09\end{array}$ & 1.1 \\
\hline
\end{tabular}


Table S1: Profiling of archazolid A.

\begin{tabular}{|c|c|c|c|c|c|c|c|c|c|}
\hline & $\begin{array}{l}\text { Test Con- } \\
\text { centration } \\
{[\mathrm{M}]}\end{array}$ & $\begin{array}{l}\% \text { Inhi- } \\
\text { bition } \\
\text { of } \\
\text { contr. } \\
\text { spec. } \\
\text { binding }\end{array}$ & $\begin{array}{l}\% \text { of } \\
\text { cific } B\end{array}$ & $\begin{array}{l}\text { Control } \\
\text { ding }\end{array}$ & pe- & $\begin{array}{l}\text { Reference } \\
\text { Compound }\end{array}$ & $\begin{array}{l}\mathrm{IC}_{50} \\
\operatorname{Ref} \\
(\mathrm{M})\end{array}$ & $\begin{array}{l}\mathrm{K}_{i} \\
\mathrm{Ref} \\
(\mathrm{M})\end{array}$ & $\begin{array}{l}\mathrm{nH} \\
\mathrm{Ref}\end{array}$ \\
\hline $\begin{array}{l}\text { P2X (agonist radioli- } \\
\text { gand) }\end{array}$ & $1 \mathrm{E}-05$ & 2 & 107.8 & 87.7 & 97.7 & $\begin{array}{l}\text { alpha ,beta - } \\
\text { MeATP }\end{array}$ & $\begin{array}{l}2.7 \mathrm{E}- \\
09\end{array}$ & $\begin{array}{l}1.2 \mathrm{E}- \\
09\end{array}$ & 0.9 \\
\hline $\begin{array}{l}\text { P2Y (agonist radioli- } \\
\text { gand) }\end{array}$ & $1 \mathrm{E}-05$ & 15 & 88.4 & 80.6 & 84.5 & dATPalpha S & $\begin{array}{l}3.5 \mathrm{E}- \\
08\end{array}$ & $\begin{array}{l}1.8 \mathrm{E}- \\
08\end{array}$ & 1.1 \\
\hline $\begin{array}{l}\text { 5-HT1A }(\mathrm{h}) \text { (agonist } \\
\text { radioligand) }\end{array}$ & $1 \mathrm{E}-05$ & 9 & 90.6 & 90.9 & 90.7 & 8-OH-DPAT & $\begin{array}{l}3.4 \mathrm{E}- \\
10\end{array}$ & $\begin{array}{l}2.1 \mathrm{E}- \\
10\end{array}$ & 0.9 \\
\hline $\begin{array}{l}\text { 5-HT1B (antagonist } \\
\text { radioligand) }\end{array}$ & $1 \mathrm{E}-05$ & 1 & 98.3 & 99.8 & 99.1 & serotonin & $\begin{array}{l}1.1 \mathrm{E}- \\
08\end{array}$ & $\begin{array}{l}6.9 \mathrm{E}- \\
09\end{array}$ & 1.7 \\
\hline $\begin{array}{l}\text { 5-HT2A (h) (antago- } \\
\text { nist radioligand) }\end{array}$ & $1 \mathrm{E}-05$ & 31 & 59.4 & 78.3 & 68.8 & ketanserin & $\begin{array}{l}7.3 \mathrm{E}- \\
10\end{array}$ & $4 \mathrm{E}-10$ & 0.9 \\
\hline $\begin{array}{l}\text { 5-HT2B (h) (agonist } \\
\text { radioligand) }\end{array}$ & $1 \mathrm{E}-05$ & -14 & 111.8 & 116.6 & 114.2 & $( \pm) \mathrm{DOI}$ & $\begin{array}{l}5.4 \mathrm{E}- \\
09\end{array}$ & $\begin{array}{l}2.7 \mathrm{E}- \\
09\end{array}$ & 0.9 \\
\hline $\begin{array}{l}\text { 5-HT2C (h) (antago- } \\
\text { nist radioligand) }\end{array}$ & $1 \mathrm{E}-05$ & -5 & 107.9 & 102.9 & 105.4 & RS 102221 & $\begin{array}{l}9.5 \mathrm{E}- \\
09\end{array}$ & $\begin{array}{l}3.2 \mathrm{E}- \\
09\end{array}$ & 17.2 \\
\hline $\begin{array}{l}\text { 5-HT3 }(\mathrm{h}) \text { (antagonist } \\
\text { radioligand) }\end{array}$ & $1 \mathrm{E}-05$ & -11 & 102.9 & 119.2 & 111.1 & MDL 72222 & $\begin{array}{l}6.5 \mathrm{E}- \\
09\end{array}$ & $\begin{array}{l}4.5 \mathrm{E}- \\
09\end{array}$ & 1.0 \\
\hline $\begin{array}{l}\text { 5-HT5a }(\mathrm{h}) \text { (agonist } \\
\text { radioligand) }\end{array}$ & $1 \mathrm{E}-05$ & 21 & 79.9 & 78.2 & 79.1 & serotonin & $\begin{array}{l}1.3 \mathrm{E}- \\
07\end{array}$ & $\begin{array}{l}6.6 \mathrm{E}- \\
08\end{array}$ & 0.8 \\
\hline $\begin{array}{l}\text { 5-HT6 (h) (agonist ra- } \\
\text { dioligand) }\end{array}$ & $1 \mathrm{E}-05$ & -9 & 104.5 & 112.9 & 108.7 & serotonin & $\begin{array}{l}1.3 \mathrm{E}- \\
07\end{array}$ & $\begin{array}{l}6.1 \mathrm{E}- \\
08\end{array}$ & 0.9 \\
\hline $\begin{array}{l}\text { 5-HT7 (h) (agonist ra- } \\
\text { dioligand) }\end{array}$ & $1 \mathrm{E}-05$ & -5 & 107.3 & 103.2 & 105.2 & serotonin & $\begin{array}{l}5.9 \mathrm{E}- \\
10\end{array}$ & $\begin{array}{l}2.2 \mathrm{E}- \\
10\end{array}$ & 0.9 \\
\hline $\begin{array}{l}\text { sigma (non-selective) } \\
\text { (h) (agonist radioli- } \\
\text { gand) }\end{array}$ & $1 \mathrm{E}-05$ & 18 & 83.2 & 81.4 & 82.3 & haloperidol & $\begin{array}{l}3.4 \mathrm{E}- \\
08\end{array}$ & $\begin{array}{l}2.7 \mathrm{E}- \\
08\end{array}$ & 0.6 \\
\hline $\begin{array}{l}\text { sst (non-selective) (ag- } \\
\text { onist radioligand) }\end{array}$ & $1 \mathrm{E}-05$ & 7 & 90.7 & 95.0 & 92.8 & $\begin{array}{l}\text { somatostatin- } \\
14\end{array}$ & $\begin{array}{l}3.3 \mathrm{E}- \\
10\end{array}$ & $2 \mathrm{E}-10$ & 1.2 \\
\hline $\begin{array}{l}\text { GR }(h) \text { (agonist radi- } \\
\text { oligand) }\end{array}$ & $1 \mathrm{E}-05$ & 15 & 80.7 & 89.7 & 85.2 & dexamethasone & $3 \mathrm{E}-09$ & $\begin{array}{l}1.5 \mathrm{E}- \\
09\end{array}$ & 1.0 \\
\hline $\begin{array}{l}\text { Platelet-Derived } \\
\text { Growth } \quad \text { Factor } \\
\text { (PDGF) }\end{array}$ & $1 \mathrm{E}-05$ & 3 & 99.3 & 94.8 & 97 & PDGF & $\begin{array}{l}1.4 \mathrm{E}- \\
10\end{array}$ & $\begin{array}{l}5.2 \mathrm{E}- \\
11\end{array}$ & 1.4 \\
\hline $\begin{array}{l}\text { VPAC1 (VIP1) (h) (ag- } \\
\text { onist radioligand) }\end{array}$ & $1 \mathrm{E}-05$ & -10 & 114.5 & 105.9 & 110.2 & VIP & $\begin{array}{l}4.8 \mathrm{E}- \\
10\end{array}$ & $\begin{array}{l}2.7 \mathrm{E}- \\
10\end{array}$ & 1.2 \\
\hline $\begin{array}{l}\text { V1a (h) (agonist radi- } \\
\text { oligand) }\end{array}$ & $1 \mathrm{E}-05$ & 0 & 104.0 & 96.8 & 100.4 & $\begin{array}{l}{[\mathrm{d}(\mathrm{CH} 2) 51, \operatorname{Tyr}(\mathrm{N}} \\
\text { AVP }\end{array}$ & $\begin{array}{l}1.3 \mathrm{E}- \\
09\end{array}$ & $8 \mathrm{E}-10$ & 1.2 \\
\hline $\begin{array}{l}\text { Ca2+ channel ( } L \text {, ve- } \\
\text { rapamil site) (pheny- } \\
\text { lalkylamine) }\end{array}$ & $1 \mathrm{E}-05$ & 52 & 46.8 & 49.9 & 48.4 & D 600 & $\begin{array}{l}1.8 \mathrm{E}- \\
08\end{array}$ & $\begin{array}{l}9.1 \mathrm{E}- \\
09\end{array}$ & 0.7 \\
\hline
\end{tabular}


Table S1: Profiling of archazolid A.

\begin{tabular}{|c|c|c|c|c|c|c|c|c|c|}
\hline & $\begin{array}{l}\text { Test Con- } \\
\text { centration } \\
{[\mathrm{M}]}\end{array}$ & $\begin{array}{l}\% \text { Inhi- } \\
\text { bition } \\
\text { of } \\
\text { contr. } \\
\text { spec. } \\
\text { binding }\end{array}$ & $\begin{array}{l}\% \text { of } \\
\text { cific } B\end{array}$ & $\begin{array}{l}\text { ontrol } \\
\text { ding }\end{array}$ & Mean & $\begin{array}{l}\text { Reference } \\
\text { Compound }\end{array}$ & $\begin{array}{l}I_{50} \\
\text { Ref } \\
(\mathrm{M})\end{array}$ & $\begin{array}{l}\mathrm{K}_{i} \\
\mathrm{Ref} \\
(\mathrm{M})\end{array}$ & $\begin{array}{l}\mathrm{nH} \\
\mathrm{Ref}\end{array}$ \\
\hline $\begin{array}{l}\text { KV channel (antago- } \\
\text { nist radioligand) }\end{array}$ & $1 \mathrm{E}-05$ & 9 & 84.0 & 98.6 & 91.3 & $\begin{array}{l}\text { alpha } \\
\text { dendrotoxin }\end{array}$ & $\begin{array}{l}2.7 \mathrm{E}- \\
10\end{array}$ & $\begin{array}{l}2.2 \mathrm{E}- \\
10\end{array}$ & 0.9 \\
\hline $\begin{array}{l}\text { SKCa channel (antag- } \\
\text { onist radioligand) }\end{array}$ & $1 \mathrm{E}-05$ & -4 & 96.1 & 112.8 & 104.5 & apamin & $\begin{array}{l}1.7 \mathrm{E}- \\
11\end{array}$ & $\begin{array}{l}8.3 \mathrm{E}- \\
12\end{array}$ & 1.7 \\
\hline $\begin{array}{l}\mathrm{Na}+\text { channel } \text { (site } 2 \text { ) } \\
\text { (antagonist radioli- } \\
\text { gand) }\end{array}$ & $1 \mathrm{E}-05$ & 39 & 63.6 & 59.4 & 61.5 & veratridine & $\begin{array}{l}5.6 \mathrm{E}- \\
06\end{array}$ & $\begin{array}{l}5.1 \mathrm{E}- \\
06\end{array}$ & 1.0 \\
\hline $\begin{array}{l}\mathrm{Cl} \text { - channel (GABA- } \\
\text { gated) (antagonist ra- } \\
\text { dioligand) }\end{array}$ & $1 \mathrm{E}-05$ & 43 & 61.5 & 52.9 & 57.2 & picrotoxinin & $\begin{array}{l}1.8 \mathrm{E}- \\
07\end{array}$ & $\begin{array}{l}1.5 \mathrm{E}- \\
07\end{array}$ & 1.0 \\
\hline $\begin{array}{l}\text { norepinephrine trans- } \\
\text { porter }(\mathrm{h}) \text { (antagonist } \\
\text { radioligand) }\end{array}$ & $1 \mathrm{E}-05$ & 16 & 89.2 & 78.2 & 83.7 & protriptyline & $\begin{array}{l}3.1 \mathrm{E}- \\
09\end{array}$ & $\begin{array}{l}2.3 \mathrm{E}- \\
09\end{array}$ & 1.2 \\
\hline $\begin{array}{l}\text { dopamine transporter } \\
\text { (h) (antagonist radioli- } \\
\text { gand) }\end{array}$ & $1 \mathrm{E}-05$ & 7 & 100.1 & 85.3 & 92.7 & ВТСР & $\begin{array}{l}1.2 \mathrm{E}- \\
08\end{array}$ & $\begin{array}{l}6.2 \mathrm{E}- \\
09\end{array}$ & 1.1 \\
\hline $\begin{array}{l}\text { 5-HT transporter } \\
\text { (h) (antagonist } \\
\text { radioligand) }\end{array}$ & $1 \mathrm{E}-05$ & -19 & 116.8 & 120.2 & 118.5 & imipramine & $3 \mathrm{E}-09$ & $\begin{array}{l}1.4 \mathrm{E}- \\
09\end{array}$ & 1.0 \\
\hline
\end{tabular}

\subsection{Kinase testing}

\section{Percent Control (\%Ctrl)}

$$
\text { Percent Control }=100 \times \frac{\text { test compound signal }- \text { positive control signal }}{\text { negative control signal }- \text { positive control signal }}
$$

with negative control $=$ DMSO $(100 \% \mathrm{Ctrl})$ and positive control $=$ control compound $(0 \% \mathrm{Ctrl})$

Table S2: Kinase screening of archazolid A (1) $(1 \mu \mathrm{M})$.

\begin{tabular}{l|c|c}
\hline DiscoveRx Gene Symbol & Entrez Gene Symbol & Percent Control [\%] \\
\hline ABL1(E255K)-phosphorylated & ABL1 & 75 \\
ABL1(T315I)-phosphorylated & ABL1 & 71 \\
ABL1-nonphosphorylated & ABL1 & 81 \\
ABL1-phosphorylated & ABL1 & 90 \\
ACVR1B & ACVR1B & 92 \\
ADCK3 & CABC1 & 100 \\
AKT1 & AKT1 & 97 \\
\hline
\end{tabular}


Table S2: Kinase screening of archazolid A (1) $(1 \mu \mathrm{M})$.

\begin{tabular}{|c|c|c|}
\hline DiscoveRx Gene Symbol & Entrez Gene Symbol & Percent Control [\%] \\
\hline AKT2 & AKT2 & 96 \\
\hline ALK & ALK & 90 \\
\hline AURKA & AURKA & 91 \\
\hline AURKB & AURKB & 89 \\
\hline$A X L$ & $A X L$ & 100 \\
\hline BMPR2 & BMPR2 & 67 \\
\hline BRAF & BRAF & 72 \\
\hline BRAF(V600E) & BRAF & 77 \\
\hline BTK & BTK & 91 \\
\hline CDK11 & CDK19 & 100 \\
\hline CDK2 & CDK2 & 100 \\
\hline CDK3 & CDK3 & 90 \\
\hline CDK7 & CDK7 & 91 \\
\hline CDK9 & CDK9 & 61 \\
\hline CHEK1 & CHEK1 & 97 \\
\hline CSF1R & CSF1R & 100 \\
\hline CSNK1D & CSNK1D & 100 \\
\hline CSNK1G2 & CSNK1G2 & 71 \\
\hline DCAMKL1 & DCLK1 & 77 \\
\hline DYRK1B & DYRK1B & 88 \\
\hline EGFR & EGFR & 88 \\
\hline EGFR(L858R) & EGFR & 91 \\
\hline EPHA2 & EPHA2 & 95 \\
\hline ERBB2 & ERBB2 & 100 \\
\hline ERBB4 & ERBB4 & 94 \\
\hline ERK1 & MAPK3 & 100 \\
\hline FAK & PTK2 & 99 \\
\hline FGFR2 & FGFR2 & 95 \\
\hline FGFR3 & FGFR3 & 98 \\
\hline FLT3 & FLT3 & 95 \\
\hline GSK3B & GSK3B & 88 \\
\hline IGF1R & IGF1R & 88 \\
\hline IKK-alpha & CHUK & 80 \\
\hline IKK-beta & IKBKB & 78 \\
\hline INSR & INSR & 88 \\
\hline JAK2(JH1domain-catalytic) & JAK2 & 68 \\
\hline JAK3(JH1domain-catalytic) & JAK3 & 78 \\
\hline JNK1 & MAPK8 & 68 \\
\hline JNK2 & MAPK9 & 71 \\
\hline JNK3 & MAPK10 & 66 \\
\hline KIT & KIT & 16 \\
\hline $\mathrm{KIT}(\mathrm{D} 816 \mathrm{~V})$ & KIT & 99 \\
\hline KIT(V559D, T670I) & KIT & 100 \\
\hline LKB1 & STK11 & 100 \\
\hline
\end{tabular}


Table S2: Kinase screening of archazolid A (1) $(1 \mu \mathrm{M})$.

\begin{tabular}{|c|c|c|}
\hline DiscoveRx Gene Symbol & Entrez Gene Symbol & Percent Control [\%] \\
\hline MAP3K4 & MAP3K4 & 98 \\
\hline MAPKAPK2 & MAPKAPK2 & 98 \\
\hline MARK3 & MARK3 & 87 \\
\hline MEK1 & MAP2K1 & 81 \\
\hline MEK2 & MAP2K2 & 85 \\
\hline MET & MET & 93 \\
\hline MKNK1 & MKNK1 & 82 \\
\hline MKNK2 & MKNK2 & 71 \\
\hline MLK1 & MAP3K9 & 95 \\
\hline p38-alpha & MAPK14 & 100 \\
\hline p38-beta & MAPK11 & 92 \\
\hline PAK1 & PAK1 & 100 \\
\hline PAK2 & PAK2 & 95 \\
\hline PAK4 & PAK4 & 81 \\
\hline PCTK1 & CDK16 & 87 \\
\hline PDGFRA & PDGFRA & 85 \\
\hline PDGFRB & PDGFRB & 66 \\
\hline PDPK1 & PDPK1 & 92 \\
\hline PIK3C2B & PIK3C2B & 100 \\
\hline PIK3CA & PIK3CA & 100 \\
\hline PIK3CG & PIK3CG & 97 \\
\hline PIM1 & PIM1 & 81 \\
\hline PIM2 & PIM2 & 100 \\
\hline PIM3 & PIM3 & 100 \\
\hline PKAC-alpha & PRKACA & 100 \\
\hline PLK1 & PLK1 & 81 \\
\hline PLK3 & PLK3 & 74 \\
\hline PLK4 & PLK4 & 87 \\
\hline PRKCE & PRKCE & 86 \\
\hline RAF1 & RAF1 & 100 \\
\hline RET & RET & 100 \\
\hline RIOK2 & RIOK2 & 82 \\
\hline ROCK2 & ROCK2 & 84 \\
\hline RSK2(Kin.Dom.1-N-terminal) & RPS6KA3 & 62 \\
\hline SNARK & NUAK2 & 72 \\
\hline SRC & SRC & 93 \\
\hline SRPK3 & SRPK3 & 100 \\
\hline TGFBR1 & TGFBR1 & 77 \\
\hline TIE2 & TEK & 100 \\
\hline TRKA & NTRK1 & 80 \\
\hline TSSK1B & TSSK1B & 99 \\
\hline TYK2(JH1domain-catalytic) & TYK2 & 80 \\
\hline ULK2 & ULK2 & 66 \\
\hline VEGFR2 & KDR & 91 \\
\hline
\end{tabular}


Table S2: Kinase screening of archazolid A (1) $(1 \mu \mathrm{M})$.

\begin{tabular}{l|c|c}
\hline DiscoveRx Gene Symbol & Entrez Gene Symbol & Percent Control [\%] \\
\hline YANK3 & STK32C & 100 \\
ZAP70 & ZAP70 & 74 \\
\hline
\end{tabular}

\subsection{MTT Assay}

Table S3: Antiproliferative activity of test compounds in 1321N1 astrocytoma cells determined by the MTT assay.

\begin{tabular}{l|c}
\hline Compound & $\begin{array}{c}\text { Growth inhibition } \\
\end{array}$ \\
\hline IC $50 \pm$ SEM $(\mathrm{nM}), \mathrm{n}=3$, duplicates (or \% growth inhibition at $10 \mu \mathrm{M})$ \\
\hline Archazolog 7 & $3050 \pm 230$ \\
Ring-opened archazolid F 8 & $0.757 \pm 0.121$ \\
Thiazole fragment 10 & $296 \pm 55$ \\
\hline
\end{tabular}

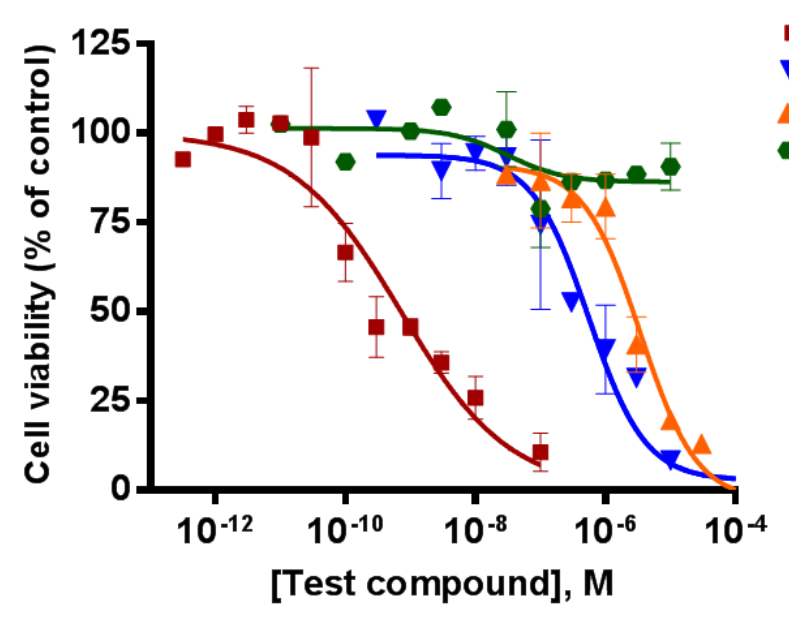

- Archazolog

V Archazolid F methyl ester

$\Delta$ Demethyl-Archazolid F

- Thiazole Fragment

Figure S3. Effects of compounds on cell growth in 1321N1 astrocytoma cells. Cells were treated with indicated concentrations of test compounds for $71 \mathrm{~h}$. The graphs show cellular growth changes in 1321N1 astrocytoma cells as assessed in MTT assays. Control cells ( $1 \%$ DMSO) were set as $100 \%$. Data points represent means \pm SEM from 3 independent experiments performed in duplicates. 


\section{5 $\mathrm{A}_{3}$ Adenosine Receptor Radioligand Binding Assay}

Table S4: $\mathrm{A}_{3}$ Adenosine receptor (human recombinant) vs. $\left[{ }^{3} \mathrm{H}\right] \mathrm{PSB} 11$

\begin{tabular}{l|c}
\hline Compound & $\mathrm{K}_{i} \pm$ SEM $(\mathrm{nM})(\mathrm{n} \geq 3)$ \\
\hline Desmethyl-archazolid F 6 & $1376 \pm 739$ \\
Archazolog 7 & $609 \pm 39$ \\
Ring-opened archazolid F8 & $1611 \pm 270$ \\
Thiazole fragment 10 & $>10,000$ \\
\hline
\end{tabular}

\subsection{Protease Assays}

Table S5: Human leukocyte elastase inhibition. $\mathrm{IC}_{50}$ values were determined from five different inhibitor concentrations in duplicates. Standard errors refer to the non-linear regression analysis.

\begin{tabular}{l|c}
\hline compound & $\mathrm{IC}_{50} \pm$ SEM (nM) \\
\hline Desmethyl-archazolid F 6 & $1540 \pm 148$ \\
Archazolog 7 & $5850 \pm 1560$ \\
Ring-opened archazolid F 8 & $2340 \pm 464$ \\
Thiazole fragment 10 & no inhibition \\
\hline
\end{tabular}

\subsection{CYP Inhibition}

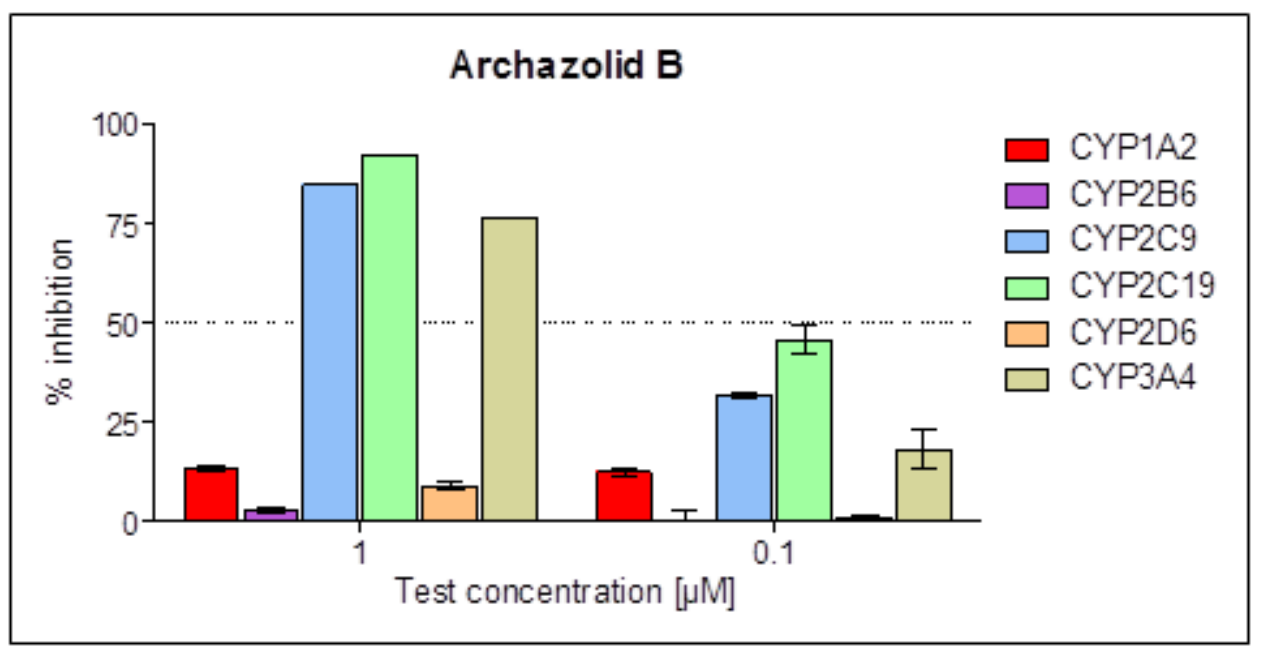

Figure S4. Cytochrome P450 enzyme inhibition. Results from 2 experiments \pm SD 
Table S6: Cytochrome P450 enzyme inhibition.

\begin{tabular}{l|c|c|c|c}
\hline & \multicolumn{4}{|c}{$\mathrm{IC}(\mu \mathrm{M})$ extrapolated from 2 test concentrations } \\
& $\mathrm{A}(\mu \mathrm{M})$ & $\mathrm{B}(\mu \mathrm{M})$ & Mean & $\mathrm{SD}$ \\
\hline CYP1A2 & $>>1$ & $>>1$ & $>>1$ & n.a. \\
CYP2B6 & $>>1$ & $>>1$ & $>>1$ & n.a. \\
CYP2C9 & 0.403 & 0.412 & 0.4 & 0.0 \\
CYP2C19 & 0.130 & 0.224 & 0.2 & 0.1 \\
CYP2D6 & $>>1$ & $>>1$ & $>>1$ & n.a. \\
CYP3A4 & 0.567 & 0.614 & 0.6 & 0.03 \\
\hline
\end{tabular}

\subsection{Inhibition by $\mathrm{P} 2 \mathrm{Y}$ and $\mathrm{P} 2 \mathrm{X}$ receptors and radioligand binding studies for human $\mathrm{P} 2 \mathrm{X} 3$ receptors}

Table S7: Inhibition of Archazolid A. [a]: $(n=3)(\%$ inhibition at $10 \mu M, n=1) h=$ human

\begin{tabular}{|c|c|}
\hline & $\mathrm{IC}_{50} \pm \mathrm{SEM}(\mu \mathrm{M})^{[a]}$ \\
\hline $\mathrm{hP} 2 \mathrm{Y}_{1}$ & $>10(1)$ \\
\hline $\mathrm{hP} 2 \mathrm{Y}_{2}$ & $>10(0)$ \\
\hline $\mathrm{hP}_{2} \mathrm{Y}_{4}$ & $\geq 10(31)$ \\
\hline $\mathrm{hP} 2 \mathrm{Y}_{6}$ & $>10(14)$ \\
\hline $\mathrm{hP} 2 \mathrm{Y}_{11}$ & $>10(-2)$ \\
\hline $\mathrm{hP} 2 \mathrm{Y}_{12}$ & $\geq 10(51)$ \\
\hline $\mathrm{hP}_{2} \mathrm{Y}_{14}$ & $>10(23)$ \\
\hline hP2X1 & $\geq 10(37)$ \\
\hline hP2X2 & $>10(-16)$ \\
\hline hP2X3 & $0.0861 \pm 0.0177$ \\
\hline $\mathrm{hP} 2 \mathrm{X} 4$ & $>10(-37)$ \\
\hline $\mathrm{hP} 2 \mathrm{X} 7$ & $>10(-23)$ \\
\hline hGPR17 & $\geq 10$ (29) \\
\hline
\end{tabular}

Table S8: $\mathrm{pEC}_{50}$ values and maximal effects for ATP in the absence and presence of different concentrations of archazolid A.

\begin{tabular}{l|c|c}
\hline ATP dose-response curve & $\mathrm{pEC}_{50}$ & Emax (\%) \\
\hline without inhibitor & 6.65 & 100 \\
$30 \mathrm{nM}$ archazolid A & 6.60 & 87 \\
$3000 \mathrm{nM}$ archazolid A & 6.82 & 57 \\
\hline
\end{tabular}




\subsection{Metabolic Stability}

These studies were performed by Pharmacelsus, Saarbrücken, Germany. ${ }^{c}$

Table S9: Metabolic stability of archazolid B and F. a: hepatic intrinsic clearance (Clint) $[\mu \mathrm{l} / \mathrm{min} / \mathrm{mg} \mathrm{protein}]$

\begin{tabular}{l|c|c}
\hline compound & Clint $^{[a]}$ & Half-live times [min] \\
\hline Archazolid B (mouse) & 495 & 2.8 \\
Archazolid B (rat) & 301 & 4.6 \\
Archazolid B (human) & 533 & 2.6 \\
Archazolid F (mouse) & 271 & 5.12 \\
\hline
\end{tabular}
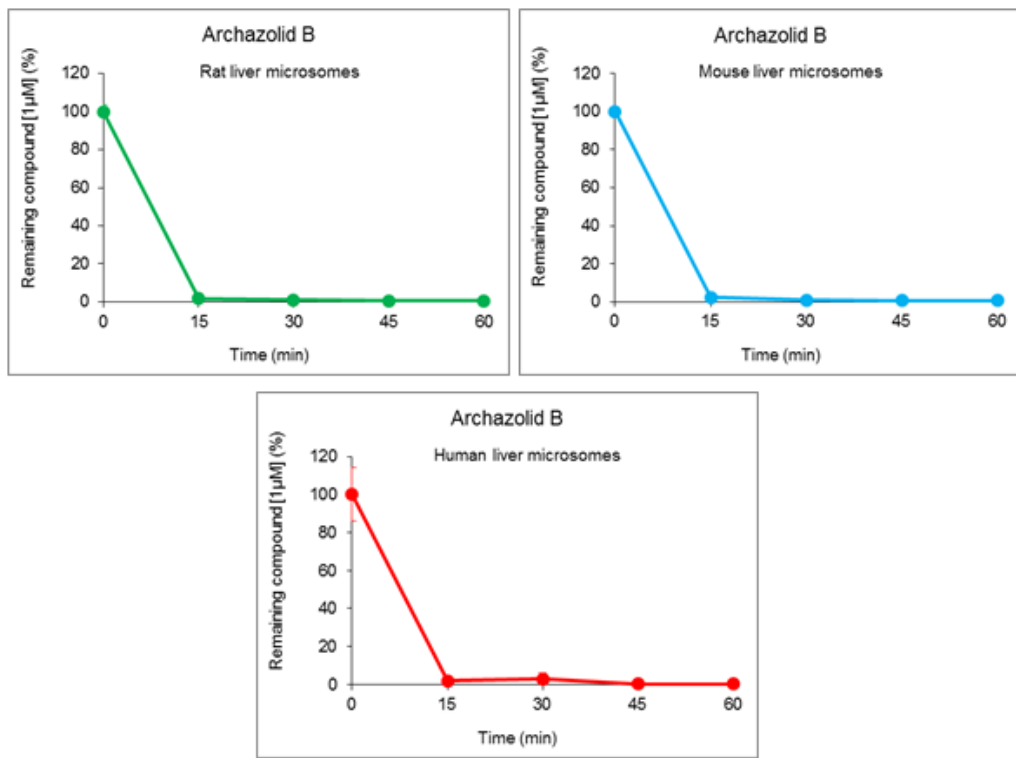

Figure S5. Metabolic stability of archazolid B in rat, mouse and human liver microsomes.

chttp://www.pharmacelsus.com/services/in-vitro-adme/ 


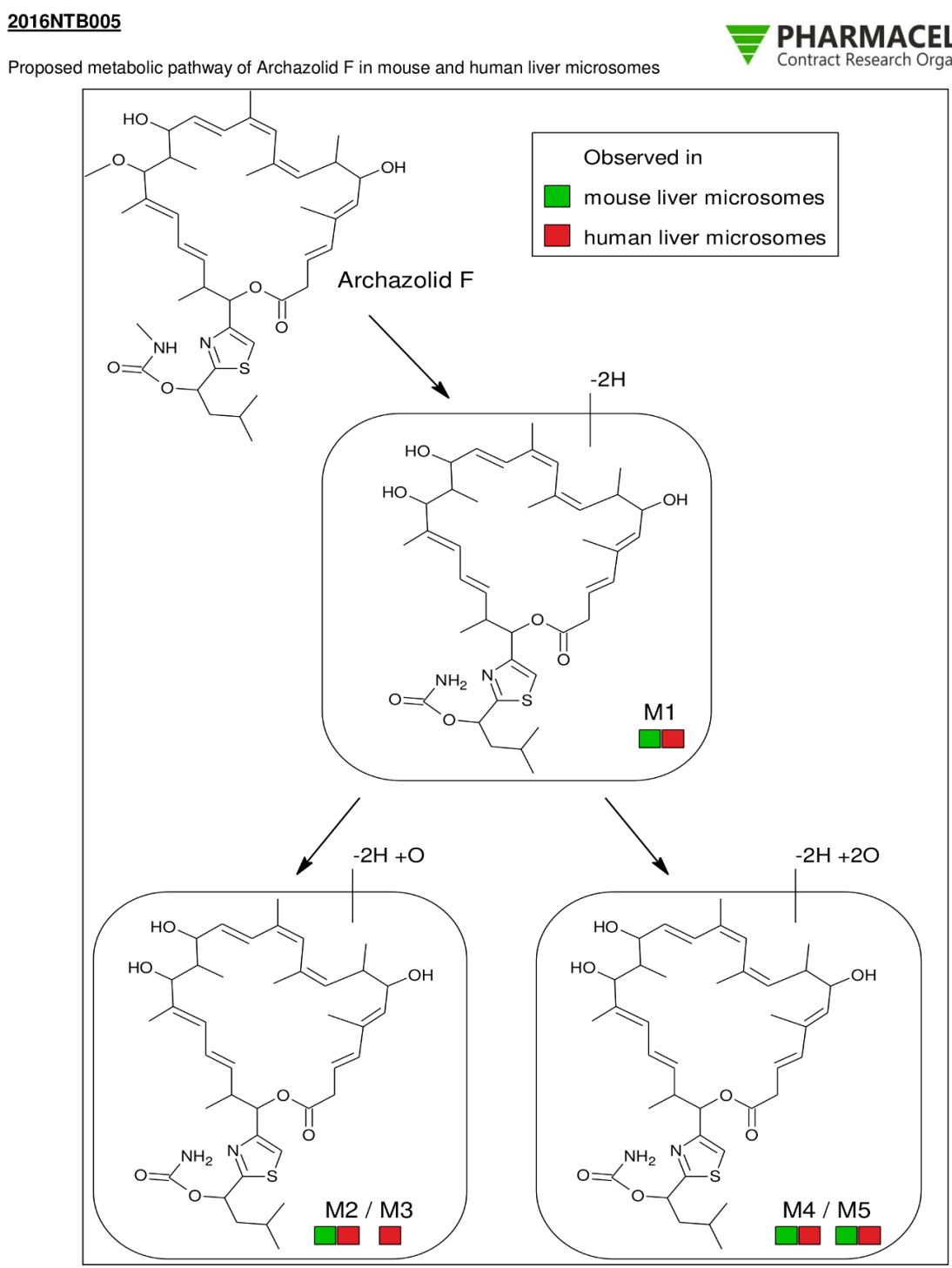

Figure S6. Proposed metabolic pathway of Archazolid F in mouse and human liver microsomes.

\subsection{In-vitro studies to determine physicochemical and pharmacokinetic properties}

These studies were performed by Pharmacelsus, Saarbrücken, Germany. http://www.pharmacelsus.com/services/invitro-adme/

\subsection{Water solubility}

Semi-thermodynamic solubility was determined by the shaking flask method. In brief, the tested compound was dissolved in DMSO at a concentration of $20 \mathrm{mM}$. This stock solution was added to 3 tubes containing PBS (cut-off concentration $200 \mu \mathrm{M}$ ) with a final solvent content of $1 \%$ DMSO. Tubes were shaken for $24 \mathrm{~h}$, undissolved particles were removed by centrifugation and the supernatant was used for quantification by LC/MS using a 5-8 point calibration 
curve. 


\section{Additional Information Chemical Synthesis}

2.1 NMR assignment 
2.1.1 Assignment for iso Archazolids $A_{1}(4)$ and $A_{2}$ (5)

Table S10: NMR assignment of iso-archazolid $\mathrm{A}_{1}(4)$ and iso-archazolid $\mathrm{A}_{2}(5)$ in $\mathrm{MeOD}$ at $500 \mathrm{MHz}, 126 \mathrm{MHz}$. a: determined by $H M Q C$ or $H M B C$.

\begin{tabular}{|c|c|c|c|c|c|c|c|c|}
\hline \multirow[b]{2}{*}{ position } & \multicolumn{4}{|c|}{ iso-archazolid $\mathrm{A}_{1}$ (4) } & \multicolumn{4}{|c|}{ iso-archazolid $\mathrm{A}_{2}$ (5) } \\
\hline & $\delta_{C}[\mathrm{ppm}]$ & $\delta_{H}[\mathrm{ppm}]$ & mult. & $J[\mathrm{~Hz}]$ & $\delta_{C}[\mathrm{ppm}]$ & $\delta_{H}[\mathrm{ppm}]$ & mult. & $J[\mathrm{~Hz}]$ \\
\hline 1 & 175.6 & - & & & 175.1 & - & & \\
\hline 2 & 44.8 & 3.27 & $\mathrm{~m}$ & & 44.6 & 3.27 & $\mathrm{~m}$ & \\
\hline 3 & 129.1 & 5.67 & dd & $8.4,15.4$ & 128.5 & 5.64 & dd & $8.3,15.5$ \\
\hline 4 & 137.7 & 6.23 & $d$ & 15.4 & 138.3 & 6.17 & $d$ & 15.5 \\
\hline 5 & 136.3 & - & & & 136.5 & - & & \\
\hline 6 & 134.9 & 5.29 & $d$ & 9.3 & 135.8 & 5.36 & $d$ & 8.9 \\
\hline 7 & 73.4 & 4.08 & dd & $9.3,9.3$ & 73.5 & 4.11 & dd & $9.4,9.4$ \\
\hline 8 & 41.7 & 2.30 & ddq & $\begin{array}{l}6.7, \quad 9.6 \\
9.6\end{array}$ & 41.3 & 2.36 & ddq & $\begin{array}{ll}7.3, & 9.3, \\
9.3 & \end{array}$ \\
\hline 9 & 132.0 & 5.18 & $d t$ & $1.4,9.9$ & 133.0 & 5.30 & $d$ & 9.5 \\
\hline 10 & 135.2 & - & & & 134.0 & - & & \\
\hline 11 & 131.1 & 5.74 & s & & 130.1 & 5.68 & $\mathrm{~s}$ & \\
\hline 12 & 133.2 & - & & & 133.1 & - & & \\
\hline 13 & 129.5 & 6.40 & $d$ & 15.9 & 129.1 & 6.75 & $d$ & 16.2 \\
\hline 14 & 133.6 & 5.81 & $\mathrm{dd}$ & $7.8,15.9$ & 133.5 & 5.74 & $\mathrm{dd}$ & $4.2,16.1$ \\
\hline 15 & n.f. & 4.11 & $\mathrm{~m}$ & & 75.7 & 4.46 & brs & \\
\hline 16 & 44.4 & 1.73 & $\mathrm{~m}$ & & 43.1 & 1.74 & $\mathrm{~m}$ & \\
\hline 17 & 90.0 & 3.20 & $d$ & 9.8 & 90.0 & 3.50 & $d$ & 9.1 \\
\hline 18 & 135.9 & - & & & 135.2 & - & & \\
\hline 19 & 129.9 & 5.73 & $d$ & 9.8 & 130.8 & 5.79 & $d$ & 10.9 \\
\hline 20 & 127.7 & 6.10 & $\mathrm{dd}$ & $10.7,15.2$ & 126.7 & 6.25 & dd & $11.1,15.0$ \\
\hline 21 & 135.4 & 5.48 & $\mathrm{dd}$ & $7.9,15.2$ & 134.8 & 5.50 & dd & $5.3,15.3$ \\
\hline 22 & 42.4 & 2.99 & ddq & $\begin{array}{ll}6.8, & 7.2 \\
7.2 & \end{array}$ & $40.4^{a}$ & 3.02 & $d t$ & $4.9,6.4$ \\
\hline 23 & 77.4 & 5.79 & $d$ & 5.8 & 77.9 & 5.81 & $d$ & 4.1 \\
\hline $\mathrm{Me} 2$ & 17.6 & 1.20 & $d$ & 6.9 & 13.2 & 1.22 & $d$ & 6.9 \\
\hline Me5 & 13.5 & 1.82 & $d$ & 1.2 & 13.2 & 1.83 & $\mathrm{~s}$ & \\
\hline Me8 & 17.9 & 0.86 & $d$ & 6.7 & 17.9 & 0.73 & $d$ & 6.8 \\
\hline Me10 & 24.9 & 1.78 & $\mathrm{~s}$ & & 24.9 & 1.76 & $\mathrm{~s}$ & \\
\hline Me12 & 20.2 & 1.92 & $d$ & 1.4 & 20.1 & 1.90 & $\mathrm{~s}$ & \\
\hline Me16 & 13.6 & 0.71 & d & 7.0 & 12.5 & 0.66 & $d$ & 7.2 \\
\hline OMe17 & 56.0 & 3.09 & $\mathrm{~s}$ & & 56.4 & 3.16 & $\mathrm{~s}$ & \\
\hline Me18 & 12.7 & 1.57 & $d$ & 1.3 & 11.6 & 1.63 & $\mathrm{~s}$ & \\
\hline Me22 & 17.9 & 1.00 & $d$ & 6.8 & 16.3 & 1.18 & $d$ & 6.9 \\
\hline $1^{\prime}$ & 73.3 & 5.99 & $\mathrm{dd}$ & $4.6,9.1$ & 73.3 & 5.98 & $\mathrm{dd}$ & $4.5,9.2$ \\
\hline $2^{\prime}$ & 173.9 & - & & & 174.0 & - & & \\
\hline $3^{\prime}$ & 155.7 & - & & & 156.1 & - & & \\
\hline $4^{\prime}$ & 117.5 & 7.27 & $\mathrm{~s}$ & & 116.0 & 7.02 & $\mathrm{~s}$ & \\
\hline $5^{\prime}$ & 46.0 & $1.79 / 1.89$ & $\mathrm{~m}$ & & 45.9 & $1.80 / 1.87$ & $\mathrm{~m}$ & \\
\hline $6^{\prime}$ & 25.8 & 1.75 & $\mathrm{~m}$ & & 25.8 & 1.75 & $\mathrm{~m}$ & \\
\hline $7^{\prime}$ & 23.3 & 0.98 & $d$ & 6.0 & 23.4 & 0.98 & $d$ & 4.8 \\
\hline
\end{tabular}


Table S10: NMR assignment of iso-archazolid $\mathrm{A}_{1}$ (4) and iso-archazolid $\mathrm{A}_{2}(5)$ in $\mathrm{MeOD}$ at $500 \mathrm{MHz}, 126 \mathrm{MHz}$. a: determined by $H M Q C$ or $H M B C$.

\begin{tabular}{|c|c|c|c|c|c|c|c|c|}
\hline \multirow[b]{2}{*}{ position } & \multicolumn{4}{|c|}{ iso-archazolid $\mathrm{A}_{1}$ (4) } & \multicolumn{4}{|c|}{ iso-archazolid $\mathrm{A}_{2}(5)$} \\
\hline & $\delta_{C}[\mathrm{ppm}]$ & $\delta_{H}[\mathrm{ppm}]$ & mult. & $J[\mathrm{~Hz}]$ & $\delta_{C}[\mathrm{ppm}]$ & $\delta_{H}[\mathrm{ppm}]$ & mult. & $J[\mathrm{~Hz}]$ \\
\hline $8^{\prime}$ & 22.3 & 0.99 & $d$ & 6.4 & 22.3 & 0.99 & $d$ & 4.9 \\
\hline $1 "$ & 158.2 & - & & & $158.3^{a}$ & - & & \\
\hline 2" & 27.5 & 2.71 & $\mathrm{~s}$ & & 27.5 & 2.71 & s & \\
\hline
\end{tabular}

\subsubsection{Assignment for Aldehyde 9}

Table S11: NMR assignment of compound 9 in $\mathrm{CD}_{2} \mathrm{Cl}_{2}$ at $700 \mathrm{MHz}$.

\begin{tabular}{l|r|c|l}
\hline position & $\delta_{H}[\mathrm{ppm}]$ & mult. & $J[\mathrm{~Hz}]$ \\
\hline 19 & 9.45 & $\mathrm{~d}$ & 7.9 \\
20 & 5.97 & $\mathrm{dd}$ & $7.3,15.7$ \\
21 & 6.92 & $\mathrm{dd}$ & $7.6,15.7$ \\
22 & 2.98 & $\mathrm{ddq}$ & $6.9,7.3,8.4$ \\
23 & 4.86 & $\mathrm{~d}$ & 5.0 \\
$1^{\prime}$ & 5.98 & $\mathrm{~d}$ & 8.4 \\
$2^{\prime}$ & - & & \\
$3^{\prime}$ & - & & \\
$4^{\prime}$ & 7.11 & $\mathrm{~s}$ & \\
$5^{\prime}$ & $1.79 / 1.86$ & $\mathrm{~m}$ & \\
$6^{\prime}$ & 1.69 & $\mathrm{~m}$ & \\
$7^{\prime}$ & 0.94 & $\mathrm{~m}$ & \\
$8^{\prime}$ & 0.94 & $\mathrm{~m}$ & \\
$1^{\prime \prime}$ & - & & \\
$2^{\prime \prime}$ & 2.77 & $\mathrm{~d}$ & 4.6 \\
Me22 & 1.07 & $\mathrm{~d}$ & 6.9 \\
NH & 4.80 & brs & \\
TES $\left(\mathrm{CH}_{2}\right)$ & 0.57 & $\mathrm{q}$ & 8.0 \\
TES $\left(\mathrm{CH}_{3}\right)$ & 0.89 & $\mathrm{t}$ & 7.9 \\
\hline
\end{tabular}

\subsubsection{Assignment for Ketone 12}

Table S12: NMR assignment of compound 12 in $\mathrm{CD}_{2} \mathrm{Cl}_{2}$ at $700 \mathrm{MHz}$.

\begin{tabular}{l|r|c|l}
\hline position & $\delta_{H}[\mathrm{ppm}]$ & mult. & $J[\mathrm{~Hz}]$ \\
\hline 3 & 4.99 & $\mathrm{~d}$ & 10.8 \\
$3^{\prime}$ & 5.14 & $\mathrm{~d}$ & 17.4 \\
\hline 4 & 6.36 & $\mathrm{ddd}$ & $0.6,10.8,17.4$ \\
5 & - & & \\
6 & 5.39 & $\mathrm{~d}$ & 9.0 \\
\hline 7 & 4.25 & $\mathrm{dd}$ & $5.6,9.0$ \\
\hline \multicolumn{3}{|c}{ continued on the next page }
\end{tabular}


Table S12: NMR assignment of compound 12 in $\mathrm{CD}_{2} \mathrm{Cl}_{2}$ at $700 \mathrm{MHz}$.

\begin{tabular}{|c|c|c|c|}
\hline position & $\delta_{H}[\mathrm{ppm}]$ & mult. & $J[\mathrm{~Hz}]$ \\
\hline 8 & 2.39 & $\mathrm{ddq}$ & $5.6,6.8,9.7$ \\
\hline 9 & 5.09 & $\mathrm{dt}$ & $1.4,9.7$ \\
\hline 10 & - & & \\
\hline 11 & 5.87 & $\mathrm{~s}$ & \\
\hline 12 & - & & \\
\hline 13 & 6.32 & $d$ & 15.8 \\
\hline 14 & 5.53 & dd & $7.6,15.9$ \\
\hline 15 & 4.22 & dd & $7.2,7.2$ \\
\hline 16 & 3.42 & $\mathrm{dq}$ & $6.9,7.0$ \\
\hline 17 & - & & \\
\hline 18 & - & & \\
\hline 19 & 6.96 & d & 10.8 \\
\hline 20 & 6.29 & dd & $11.0,15.2$ \\
\hline 21 & 6.10 & dd & $8.5,15.1$ \\
\hline 22 & 2.82 & $\mathrm{ddq}$ & $4.8,6.8,7.4$ \\
\hline 23 & 4.79 & $d$ & 4.8 \\
\hline $1^{\prime}$ & 5.98 & dd & $5.3,8.6$ \\
\hline $2^{\prime}$ & - & & \\
\hline $3^{\prime}$ & - & & \\
\hline $4^{\prime}$ & 7.07 & $\mathrm{~s}$ & \\
\hline $5^{\prime}$ & $1.80 / 1.87$ & $\mathrm{~m}$ & \\
\hline $6^{\prime}$ & 1.70 & $\mathrm{~m}$ & \\
\hline $7^{\prime}$ & 0.95 & $\mathrm{~m}$ & \\
\hline $8^{\prime}$ & 0.95 & $\mathrm{~m}$ & \\
\hline $1 "$ & - & & \\
\hline $2 "$ & 2.77 & $d$ & 4.9 \\
\hline Me5 & 1.71 & $d$ & 1.2 \\
\hline Me8 & 0.88 & $d$ & 6.7 \\
\hline Me10 & 1.76 & $\mathrm{~s}$ & \\
\hline Me12 & 1.76 & $\mathrm{~s}$ & \\
\hline Me16 & 1.08 & $d$ & 6.7 \\
\hline Me18 & 1.72 & $\mathrm{~s}$ & \\
\hline Me22 & 1.04 & $d$ & 6.9 \\
\hline $\mathrm{NH}$ & 4.78 & brs & \\
\hline TES $\left(\mathrm{CH}_{2}\right)$ & 0.56 & $q$ & 8.0 \\
\hline TES $\left(\mathrm{CH}_{3}\right)$ & 0.88 & $\mathrm{t}$ & 8.0 \\
\hline TBS (tBu) & $0.87,0.85$ & $\mathrm{~s}, \mathrm{~s}$ & \\
\hline TBS (Me) & $0.02,0.00,-0.03,-0.05$ & $s, s, s, s$ & \\
\hline
\end{tabular}

\subsubsection{Assignment for Alcohol 13}


Table S13: NMR assignment of compound 13 in $\mathrm{CD}_{2} \mathrm{Cl}_{2}$ at $700 \mathrm{MHz}$.

\begin{tabular}{|c|c|c|c|}
\hline position & $\delta_{H}[\mathrm{ppm}]$ & mult. & $J[\mathrm{~Hz}]$ \\
\hline 1 & - & & \\
\hline 2 & 3.10 & ddt & $1.4,2.9,6.9$ \\
\hline 3 & 5.89 & $\mathrm{~m}$ & \\
\hline 4 & 5.14 & $\mathrm{~m}$ & \\
\hline $4^{\prime}$ & 5.14 & $\mathrm{~m}$ & \\
\hline 3 & 4.99 & $d$ & 10.8 \\
\hline $3^{\prime}$ & 5.14 & $\mathrm{~m}$ & \\
\hline 4 & 6.36 & ddd & $0.8,10.8,17.4$ \\
\hline 5 & - & & \\
\hline 6 & 5.39 & $d$ & 8.9 \\
\hline 7 & 4.26 & dd & $5.7,9.0$ \\
\hline 8 & 2.43 & ddq & $5.7,6.7,9.1$ \\
\hline 9 & 5.08 & $d t$ & $1.3,9.7$ \\
\hline 10 & - & & \\
\hline 11 & 5.89 & $\mathrm{~s}$ & \\
\hline 12 & - & & \\
\hline 13 & 6.45 & $d$ & 15.8 \\
\hline 14 & 5.77 & dd & $8.0,15.2$ \\
\hline 15 & 4.42 & dd & $2.1,7.6$ \\
\hline 16 & 1.86 & $\mathrm{~m}$ & \\
\hline 17 & 3.88 & dd & $2.0,9.3$ \\
\hline 18 & - & & \\
\hline 19 & 5.89 & $d$ & 10.8 \\
\hline 20 & 6.23 & dd & $10.8,15.0$ \\
\hline 21 & 5.53 & dd & $8.5,15.1$ \\
\hline 22 & 2.98 & $\mathrm{~m}$ & \\
\hline 23 & 5.77 & $d$ & 6.9 \\
\hline $1^{\prime}$ & 5.99 & dd & $5.5,9.1$ \\
\hline $2^{\prime}$ & - & & \\
\hline $3^{\prime}$ & - & & \\
\hline $4^{\prime}$ & 7.11 & s & \\
\hline $5^{\prime}$ & $1.81 / 1.86$ & $\mathrm{~m}$ & \\
\hline $6^{\prime}$ & 1.81 & $\mathrm{~m}$ & \\
\hline $7^{\prime}$ & 0.96 & $d$ & 6.7 \\
\hline $8^{\prime}$ & 0.96 & $d$ & 6.7 \\
\hline $1 "$ & - & & \\
\hline 2" & 2.77 & $d$ & 4.8 \\
\hline Me5 & 1.71 & $d$ & 1.2 \\
\hline Me8 & 0.86 & d & 7.0 \\
\hline Me10 & 1.78 & $\mathrm{~s}$ & \\
\hline Me12 & 1.66 & $\mathrm{~s}$ & \\
\hline Me16 & 0.63 & $d$ & 7.0 \\
\hline Me18 & 1.88 & $d$ & 1.3 \\
\hline Me22 & 0.95 & d & 7.1 \\
\hline
\end{tabular}


Table S13: NMR assignment of compound 13 in $\mathrm{CD}_{2} \mathrm{Cl}_{2}$ at $700 \mathrm{MHz}$.

\begin{tabular}{l|r|c|c}
\hline position & $\delta_{H}[\mathrm{ppm}]$ & mult. & $J[\mathrm{~Hz}]$ \\
\hline $\mathrm{NH}$ & 4.83 & brs & \\
OH & 3.79 & $\mathrm{~d}$ & 2.0 \\
TBS (tBu) & $0.91,0.85$ & $\mathrm{~s}, \mathrm{~s}$ & \\
TBS (Me) & $0.09,0.04,0.00,-0.04$ & $\mathrm{~s}, \mathrm{~s}, \mathrm{~s}, \mathrm{~s}$ & \\
\hline
\end{tabular}

\subsubsection{Assignment for Desmethylarchazolid F (6)}

Table S14: NMR assignment of compound 6 in $\mathrm{CD}_{2} \mathrm{Cl}_{2}$ at $700 \mathrm{MHz}, 176 \mathrm{MHz}$.

\begin{tabular}{|c|c|c|c|c|}
\hline position & $\delta_{C}[\mathrm{ppm}]$ & $\delta_{H}[\mathrm{ppm}]$ & mult. & $J[\mathrm{~Hz}]$ \\
\hline 1 & 171.0 & - & & \\
\hline 2 & 37.8 & $3.07 / 3.01$ & $\mathrm{ddd} / \mathrm{ddd}$ & $1.8,4.9,16.0 / 1.1,7.5,15.8$ \\
\hline 3 & 121.6 & 5.69 & ddd & $4.9,7.4,16.0$ \\
\hline 4 & 137.6 & 6.09 & $d$ & 15.9 \\
\hline 5 & 137.8 & - & & \\
\hline 6 & 132.1 & 5.24 & $d$ & 8.8 \\
\hline 7 & 73.4 & 4.10 & dd & $9.6,9.6$ \\
\hline 8 & 41.4 & 2.32 & ddq & $6.8,6.8,9.8$ \\
\hline 9 & 130.6 & 5.27 & $d t$ & $1.3,9.6$ \\
\hline 10 & 137.9 & - & & \\
\hline 11 & 128.7 & 5.77 & $\mathrm{~s}$ & \\
\hline 12 & 133.5 & - & & \\
\hline 13 & 127.6 & 6.63 & dd & $1.5,15.9$ \\
\hline 14 & 131.3 & 5.92 & dd & $3.6,15.9$ \\
\hline 15 & 76.8 & 4.36 & brs & \\
\hline 16 & 40.1 & 2.5 & ddq & $3.7,7.4,11.1$ \\
\hline 17 & 81.2 & 3.88 & dd & $3.0,10.2$ \\
\hline 18 & 138.2 & - & & \\
\hline 19 & 127.3 & 5.80 & $d$ & 10.6 \\
\hline 20 & 128.2 & 6.26 & dd & $10.8,15.1$ \\
\hline 21 & 136.1 & 5.54 & dd & $9.4,15.0$ \\
\hline 22 & 42.7 & 3.01 & $\mathrm{~m}$ & \\
\hline 23 & 75.7 & 5.73 & $d$ & 9.3 \\
\hline $1^{\prime}$ & 72.8 & 6.00 & dd & $5.0,9.2$ \\
\hline $2^{\prime}$ & 172.5 & - & & \\
\hline $3^{\prime}$ & 154.3 & - & & \\
\hline $4^{\prime}$ & 118.0 & 7.14 & $\mathrm{~s}$ & \\
\hline $5^{\prime}$ & 45.1 & $1.80 / 1.89$ & $\mathrm{~m}$ & \\
\hline $6^{\prime}$ & 25.2 & 1.71 & $\mathrm{~m}$ & \\
\hline $7^{\prime}$ & 22.4 & 0.97 & $d$ & 6.5 \\
\hline $8^{\prime}$ & 23.3 & 0.96 & $d$ & 6.6 \\
\hline $1 "$ & 156.4 & - & & \\
\hline $2 "$ & 28.0 & 2.78 & $d$ & 4.8 \\
\hline Me5 & 13.6 & 1.76 & $d$ & 1.1 \\
\hline
\end{tabular}

continued on the next page 
Table S14: NMR assignment of compound 6 in $\mathrm{CD}_{2} \mathrm{Cl}_{2}$ at $700 \mathrm{MHz}, 176 \mathrm{MHz}$.

\begin{tabular}{l|r|r|r|l}
\hline position & $\delta_{C}[\mathrm{ppm}]$ & $\delta_{H}[\mathrm{ppm}]$ & mult. & $J[\mathrm{~Hz}]$ \\
\hline $\mathrm{Me} 8$ & 17.2 & 0.79 & $\mathrm{~d}$ & 6.8 \\
$\mathrm{Me} 10$ & 25.0 & 1.83 & $\mathrm{~s}$ & \\
$\mathrm{Me} 12$ & 20.5 & 1.92 & $\mathrm{~d}$ & 1.3 \\
$\mathrm{Me} 16$ & 14.8 & 0.67 & $\mathrm{~d}$ & 7.3 \\
$\mathrm{Me} 18$ & 11.1 & 1.67 & $\mathrm{~d}$ & 1.1 \\
$\mathrm{Me} 22$ & 18.3 & 0.86 & $\mathrm{~d}$ & 6.7 \\
$\mathrm{OH} 7$ & - & 2.43 & $\mathrm{~s}$ & \\
$\mathrm{OH} 15$ & - & 4.83 & brs & \\
$\mathrm{OH} 17$ & - & 4.68 & brs & \\
$\mathrm{NH}$ & - & 4.83 & brs & \\
\hline
\end{tabular}

\subsubsection{Assignment for Ketone 18}

Table S15: NMR assignment of compound 18 in $\mathrm{CD}_{2} \mathrm{Cl}_{2}$ at $700 \mathrm{MHz}$.

\begin{tabular}{|c|c|c|c|}
\hline position & $\delta_{H}[\mathrm{ppm}]$ & mult. & $J[\mathrm{~Hz}]$ \\
\hline 3 & 4.99 & $d$ & 10.8 \\
\hline $3^{\prime}$ & 5.14 & $d$ & 17.5 \\
\hline 4 & 6.37 & ddd & $0.7,10.7,17.3$ \\
\hline 5 & - & & \\
\hline 6 & 5.39 & $d$ & 9.0 \\
\hline 7 & 4.25 & $\mathrm{dd}$ & $5.6,9.0$ \\
\hline 8 & 2.38 & $\mathrm{ddq}$ & $5.6,6.5,9.7$ \\
\hline 9 & 5.09 & $\mathrm{dt}$ & $1.4,9.7$ \\
\hline 10 & - & & \\
\hline 11 & 5.87 & $\mathrm{~s}$ & \\
\hline 12 & - & & \\
\hline 13 & 6.33 & d & 15.6 \\
\hline 14 & 5.55 & $\mathrm{dd}$ & $7.7,15.6$ \\
\hline 15 & 4.24 & $d d$ & $7.1,7.1$ \\
\hline 16 & 3.42 & $\mathrm{dq}$ & $6.8,6.8$ \\
\hline 17 & - & & \\
\hline 18 & - & & \\
\hline 19 & 6.99 & d & 11.1 \\
\hline 20 & 6.50 & $d d t$ & $1.4,10.9,15.0$ \\
\hline 21 & 6.13 & $\mathrm{dt}$ & $7.2,14.7$ \\
\hline 22 & 2.41 & $\mathrm{dt}$ & $6.5,6.5$ \\
\hline 23 & 3.71 & $\mathrm{t}$ & 6.4 \\
\hline Me5 & 1.71 & d & 1.2 \\
\hline Me8 & 0.88 & d & 6.8 \\
\hline Me10 & 1.76 & $\mathrm{~s}$ & \\
\hline Me12 & 1.76 & $\mathrm{~s}$ & \\
\hline Me16 & 1.09 & d & 6.8 \\
\hline Me18 & 1.78 & $\mathrm{~s}$ & \\
\hline
\end{tabular}


Table S15: NMR assignment of compound 18 in $\mathrm{CD}_{2} \mathrm{Cl}_{2}$ at $700 \mathrm{MHz}$.

\begin{tabular}{l|r|c|c}
\hline position & $\delta_{H}[\mathrm{ppm}]$ & mult. & $J[\mathrm{~Hz}]$ \\
\hline TBS $(\mathrm{tBu})$ & $0.89,0.87,0.85$ & $\mathrm{~s}, \mathrm{~s}$ & \\
TBS $(\mathrm{Me})$ & $0.06,0.02,0.00,-0.02,-0.05$ & $\mathrm{~s}, \mathrm{~s}, \mathrm{~s}, \mathrm{~s}$ & \\
\hline
\end{tabular}

\subsubsection{Assignment for Alcohol 22}

Table S16: NMR assignment of compound 22 in $\mathrm{CD}_{2} \mathrm{Cl}_{2}$ at $500 \mathrm{MHz}$.

\begin{tabular}{|c|c|c|c|}
\hline position & $\delta_{H}[\mathrm{ppm}]$ & mult. & $J[\mathrm{~Hz}]$ \\
\hline 3 & 4.99 & $d$ & 10.8 \\
\hline $3^{\prime}$ & 5.14 & $d$ & 17.5 \\
\hline 4 & 6.36 & dd & $10.7,17.3$ \\
\hline 5 & - & & \\
\hline 6 & 5.39 & $d$ & 8.9 \\
\hline 7 & 4.26 & dd & $5.6,9.0$ \\
\hline 8 & 2.43 & ddq & $5.6,6.7,9.6$ \\
\hline 9 & 5.09 & $d t$ & $1.3,9.7$ \\
\hline 10 & - & & \\
\hline 11 & 5.90 & $\mathrm{~s}$ & \\
\hline 12 & - & & \\
\hline 13 & 6.45 & $d$ & 15.8 \\
\hline 14 & 5.78 & dd & $7.6,15.8$ \\
\hline 15 & 4.43 & dd & $2.2,7.1$ \\
\hline 16 & 1.86 & $\mathrm{~m}$ & \\
\hline 17 & 3.89 & $d$ & 8.0 \\
\hline 18 & - & & \\
\hline 19 & 5.92 & $d$ & 10.8 \\
\hline 20 & 6.32 & ddt & $1.3,10.9,15.0$ \\
\hline 21 & 5.65 & $d t$ & $7.1,14.7$ \\
\hline 22 & 2.31 & $d t$ & $6.7,6.7$ \\
\hline 23 & 3.65 & $d t$ & $1.0,6.6$ \\
\hline Me5 & 1.71 & $d$ & 1.2 \\
\hline Me8 & 0.87 & $d$ & 6.9 \\
\hline Me10 & 1.78 & $\mathrm{~s}$ & \\
\hline Me12 & 1.69 & $\mathrm{~s}$ & \\
\hline Me16 & 0.65 & $d$ & 7.1 \\
\hline Me18 & 1.89 & $d$ & 1.3 \\
\hline $\mathrm{OH}$ & 3.79 & $d$ & 2.0 \\
\hline TBS (tBu) & $0.91,0.89,0.86$ & $s, s$ & \\
\hline TBS (Me) & $0.09,0.04,0.00,-0.04$ & $s, s, s, s$ & \\
\hline
\end{tabular}

\subsubsection{Assignment for Methyl Ether 19}


Table S17: NMR assignment of compound 19 in $\mathrm{CD}_{2} \mathrm{Cl}_{2}$ at $700 \mathrm{MHz}$.

\begin{tabular}{|c|c|c|c|}
\hline position & $\delta_{H}[\mathrm{ppm}]$ & mult. & $J[\mathrm{~Hz}]$ \\
\hline 3 & 4.99 & $d$ & 10.8 \\
\hline $3^{\prime}$ & 5.14 & $d$ & 17.7 \\
\hline 4 & 6.36 & ddd & $0.8,10.8,17.4$ \\
\hline 5 & - & & \\
\hline 6 & 5.40 & $d$ & 9.0 \\
\hline 7 & 4.25 & dd & $5.6,9.0$ \\
\hline 8 & 2.43 & ddq & $5.6,6.7,9.7$ \\
\hline 9 & 5.07 & $d t$ & $1.4,9.7$ \\
\hline 10 & - & & \\
\hline 11 & 5.84 & $\mathrm{~s}$ & \\
\hline 12 & - & & \\
\hline 13 & 6.42 & $d$ & 15.9 \\
\hline 14 & 5.72 & $\mathrm{dd}$ & $7.0,15.7$ \\
\hline 15 & 4.69 & dd & $1.6,6.9$ \\
\hline 16 & 1.58 & $\mathrm{~m}$ & \\
\hline 17 & 3.34 & $d$ & 9.9 \\
\hline 18 & - & & \\
\hline 19 & 5.91 & d & 10.8 \\
\hline 20 & 6.35 & $d d t$ & $1.4,10.9,15.1$ \\
\hline 21 & 5.68 & $\mathrm{dt}$ & $7.2,14.7$ \\
\hline 22 & 2.32 & $\mathrm{dt}$ & $6.6,6.6$ \\
\hline 23 & 3.66 & $\mathrm{t}$ & $1.0,6.6$ \\
\hline Me5 & 1.71 & $d$ & 1.2 \\
\hline Me8 & 0.88 & $d$ & 6.8 \\
\hline Me10 & 1.79 & $\mathrm{~s}$ & \\
\hline Me12 & 1.57 & $\mathrm{~s}$ & \\
\hline Me16 & 0.63 & $d$ & 7.0 \\
\hline OMe17 & 3.10 & $\mathrm{~s}$ & \\
\hline Me18 & 1.84 & $d$ & 1.3 \\
\hline TBS (tBu) & $0.92,0.89,0.85$ & $\mathrm{~s}, \mathrm{~s}$ & \\
\hline TBS (Me) & $0.05,0.05,0.00,-0.05$ & $\mathrm{~s}, \mathrm{~s}, \mathrm{~s}, \mathrm{~s}$ & \\
\hline
\end{tabular}

\subsubsection{Assignment for Alcohol 23}

Table S18: NMR assignment of compound 23 in $\mathrm{CD}_{2} \mathrm{Cl}_{2}$ at $700 \mathrm{MHz}$.

\begin{tabular}{l|r|c|l}
\hline position & $\delta_{H}[\mathrm{ppm}]$ & mult. & $J[\mathrm{~Hz}]$ \\
\hline 3 & 4.99 & $\mathrm{~d}$ & 10.7 \\
3 & 5.14 & $\mathrm{~d}$ & 17.4 \\
\hline 4 & 6.37 & $\mathrm{ddd}$ & $0.7,10.7,17.4$ \\
5 & - & & \\
6 & 5.40 & $\mathrm{~d}$ & 9.1 \\
7 & 4.25 & $\mathrm{dd}$ & $5.6,9.0$ \\
\hline
\end{tabular}

continued on the next page 
Table S18: NMR assignment of compound 23 in $\mathrm{CD}_{2} \mathrm{Cl}_{2}$ at $700 \mathrm{MHz}$.

\begin{tabular}{|c|c|c|c|}
\hline position & $\delta_{H}[\mathrm{ppm}]$ & mult. & $J[\mathrm{~Hz}]$ \\
\hline 8 & 2.43 & ddq & $5.6,6.7,9.7$ \\
\hline 9 & 5.07 & $\mathrm{dt}$ & $1.4,9.7$ \\
\hline 10 & - & & \\
\hline 11 & 5.84 & s & \\
\hline 12 & - & & \\
\hline 13 & 6.42 & $d$ & 15.9 \\
\hline 14 & 5.72 & dd & $6.9,15.9$ \\
\hline 15 & 4.69 & $d$ & 6.9 \\
\hline 16 & 1.60 & $\mathrm{~m}$ & \\
\hline 17 & 3.35 & $d$ & 9.9 \\
\hline 18 & - & & \\
\hline 19 & 5.93 & $d$ & 10.8 \\
\hline 20 & 6.40 & $d d t$ & $1.3,10.8,15.0$ \\
\hline 21 & 5.67 & $\mathrm{dt}$ & $7.2,14.7$ \\
\hline 22 & 2.36 & $d t$ & $6.3,6.3$ \\
\hline 23 & 3.65 & $d t$ & $5.8,6.3$ \\
\hline Me5 & 1.71 & $d$ & 1.2 \\
\hline Me8 & 0.88 & $d$ & 6.8 \\
\hline Me10 & 1.79 & $\mathrm{~s}$ & \\
\hline Me12 & 1.58 & s & \\
\hline Me16 & 0.63 & $d$ & 7.0 \\
\hline OMe17 & 3.10 & $\mathrm{~s}$ & \\
\hline Me18 & 1.84 & $d$ & 1.3 \\
\hline $\mathrm{OH}$ & 1.40 & $\mathrm{t}$ & 5.8 \\
\hline TBS (tBu) & $0.92,0.85$ & $\mathrm{~s}, \mathrm{~s}$ & \\
\hline TBS (Me) & $0.05,0.00,-0.04$ & $s, s, s$ & \\
\hline
\end{tabular}

\subsubsection{Assignment for Ester 20}

Table S19: NMR assignment of compound 20 in $\mathrm{CD}_{2} \mathrm{Cl}_{2}$ at $500 \mathrm{MHz}$.

\begin{tabular}{l|r|c|l}
\hline position & $\delta_{H}[\mathrm{ppm}]$ & mult. & $J[\mathrm{~Hz}]$ \\
\hline 1 & - & & \\
2 & 3.08 & $\mathrm{dt}$ & $1.4,6.9$ \\
\hline 3 & 5.92 & $\mathrm{ddt}$ & $6.9,10.0,17.2$ \\
4 & $5.13-5.17$ & $\mathrm{~m}$ & \\
\hline 3 & 4.99 & $\mathrm{~d}$ & 10.8 \\
\hline 3 & 5.14 & $\mathrm{~d}$ & 17.5 \\
\hline 4 & 6.36 & $\mathrm{ddd}$ & $0.6,10.7,17.4$ \\
5 & - & & \\
\hline 7 & 5.40 & $\mathrm{~d}$ & 8.8 \\
8 & 4.25 & $\mathrm{dd}$ & $5.6,9.0$ \\
9 & 2.42 & $\mathrm{~m}$ & \\
\hline \multicolumn{4}{|c|}{ continued on the next page }
\end{tabular}


Table S19: NMR assignment of compound 20 in $\mathrm{CD}_{2} \mathrm{Cl}_{2}$ at $500 \mathrm{MHz}$.

\begin{tabular}{|c|c|c|c|}
\hline position & $\delta_{H}[\mathrm{ppm}]$ & mult. & $J[\mathrm{~Hz}]$ \\
\hline 10 & - & & \\
\hline 11 & 5.84 & $\mathrm{~s}$ & \\
\hline 12 & - & & \\
\hline 13 & 6.42 & $d$ & 15.1 \\
\hline 14 & 5.72 & $\mathrm{dd}$ & $6.7,15.9$ \\
\hline 15 & 4.69 & d & 6.9 \\
\hline 16 & 1.59 & $\mathrm{~m}$ & \\
\hline 17 & 3.35 & $\mathrm{~d}$ & 9.9 \\
\hline 18 & - & & \\
\hline 19 & 5.92 & $d$ & 10.8 \\
\hline 20 & 6.38 & $\mathrm{ddt}$ & $1.4,10.8,15.2$ \\
\hline 21 & 5.64 & $\mathrm{dt}$ & $7.0,14.6$ \\
\hline 22 & 2.44 & $d t$ & $6.7,6.7$ \\
\hline 23 & 4.12 & $\mathrm{t}$ & 6.7 \\
\hline Me5 & 1.71 & $d$ & 1.2 \\
\hline Me8 & 0.88 & $\mathrm{~d}$ & 6.9 \\
\hline Me10 & 1.79 & s & \\
\hline Me12 & 1.58 & $\mathrm{~s}$ & \\
\hline Me16 & 0.63 & d & 7.0 \\
\hline OMe17 & 3.10 & $\mathrm{~s}$ & \\
\hline Me18 & 1.84 & $d$ & 1.3 \\
\hline TBS $(\mathrm{tBu})$ & $0.92,0.85$ & $s, s$ & \\
\hline TBS $(\mathrm{Me})$ & $0.05,0.00,-0.04$ & $\mathrm{~s}, \mathrm{~s}, \mathrm{~s}$ & \\
\hline
\end{tabular}

\subsubsection{Assignment for Archazolog (7)}

Table S20: NMR assignment of compound 7 in $\mathrm{CD}_{2} \mathrm{Cl}_{2}$ at $700 \mathrm{MHz}, 176 \mathrm{MHz}$. a: determined by $H M Q C$ or $H M B C$.

\begin{tabular}{l|r|r|c|l}
\hline position & $\delta_{C}[\mathrm{ppm}]$ & $\delta_{H}[\mathrm{ppm}]$ & mult. & $J[\mathrm{~Hz}]$ \\
\hline 1 & 172.0 & - & & \\
2 & 39.0 & $2.98 / 3.08$ & $\mathrm{dd} / \mathrm{dd}$ & $8.7,15.3 / 6.5,15.3$ \\
3 & 121.9 & 5.73 & $\mathrm{ddd}$ & $6.1,8.7,15.3$ \\
4 & 138.2 & 6.01 & $\mathrm{~d}$ & 15.5 \\
5 & 137.1 & - & & \\
6 & 133.8 & 5.27 & $\mathrm{~d}$ & 8.5 \\
7 & 73.2 & 4.07 & $\mathrm{ddd}$ & $2.8,9.2,9.8$ \\
8 & 41.0 & 2.33 & $\mathrm{ddq}$ & $6.7,9.8,9.8$ \\
9 & 131.2 & 5.21 & $\mathrm{dt}$ & $1.1,9.9$ \\
10 & $135.3^{a}$ & - & & \\
11 & 129.0 & 5.63 & $\mathrm{~s}$ & \\
12 & 132.5 & - & & \\
13 & 128.0 & 6.56 & $\mathrm{~d}$ & 16.1 \\
\hline 14 & 133.8 & 5.70 & $\mathrm{dd}$ & $4.6,16.1$ \\
15 & $73.5^{a}$ & 4.44 & brs & \\
\hline
\end{tabular}

continued on the next page 
Table S20: NMR assignment of compound 7 in $\mathrm{CD}_{2} \mathrm{Cl}_{2}$ at $700 \mathrm{MHz}, 176 \mathrm{MHz}$. a: determined by $\mathrm{HMQC}$ or $\mathrm{HMBC}$.

\begin{tabular}{l|r|r|c|l}
\hline position & $\delta_{C}[\mathrm{ppm}]$ & $\delta_{H}[\mathrm{ppm}]$ & mult. & $J[\mathrm{~Hz}]$ \\
\hline 16 & 41.9 & 1.82 & $\mathrm{~m}$ & \\
17 & 89.5 & 3.44 & $\mathrm{~d}$ & 9.6 \\
18 & 134.6 & - & & \\
19 & 129.9 & 5.88 & $\mathrm{~d}$ & 11.4 \\
20 & 128.5 & 6.33 & $\mathrm{ddt}$ & $1.3,10.8,15.1$ \\
21 & 131.3 & 5.55 & $\mathrm{dt}$ & $7.2,15.1$ \\
22 & 33.2 & 2.45 & $\mathrm{~m}$ & \\
23 & 63.9 & $4.37 / 3.99$ & $\mathrm{ddd} / \mathrm{ddd}$ & $5.4,8.1,10.9 / 4.4,4.4,10.9$ \\
Me5 & 13.4 & 1.80 & $\mathrm{~d}$ & 1.3 \\
Me8 & 17.9 & 0.71 & $\mathrm{~d}$ & 6.8 \\
Me10 & 25.1 & 1.78 & $\mathrm{~s}$ & \\
Me12 & 20.4 & 1.90 & $\mathrm{~d}$ & 1.3 \\
Me16 & $12.4^{a}$ & 0.54 & $\mathrm{~d}$ & 7.2 \\
OMe17 & 56.3 & 3.16 & $\mathrm{~s}$ & \\
Me18 & 10.9 & 1.62 & $\mathrm{~d}$ & 1.1 \\
OH7 & - & 2.11 & brs & \\
OH15 & - & 3.05 & $\mathrm{~d}$ & 9.9 \\
\hline & &
\end{tabular}

\subsection{HPLC configuration for semipreparative separation}

Semi-preparative and analytical HPLC analyses were performed on Knauer Wissenschaftliche Geräte GmbH systems by Andreas Schneider. The solvents for HPLC were purchased in HPLC grade. The chromatograms were recorded by UV-detection at $240 \mathrm{~nm}$.

Table S21: HPLC configuration for analytical HPLC.

\begin{tabular}{|c|c|c|}
\hline & System A & System C \\
\hline series & PLATINblue & PLATINblue \\
\hline pumps & binary, HPG P1 system, $10 \mathrm{~mL}$ & binary, HPG P1 system, $5 \mathrm{~mL}$ \\
\hline pressure & 750 bar & 1000 bar \\
\hline autosampler & AS1 with $10 \mu \mathrm{L}$ injection loop & AS1 with $10 \mu \mathrm{L}$ injection loop \\
\hline mixing chamber & static, SmartMix $350 \mu \mathrm{L}$ & static, SmartMix $100 \mu \mathrm{L}$ \\
\hline column heater & $\mathrm{T} 1$ & $\mathrm{~T} 1$ \\
\hline detection type & $\begin{array}{l}\text { PDA UV/VIS detection PDA1, } \mathrm{D}_{2} / \mathrm{Hg} \text { halo- } \\
\text { gen lamps, } 190-1000 \mathrm{~nm}\end{array}$ & $\begin{array}{l}\text { PDA UV/VIS detection PDA1, } \mathrm{D}_{2} / \mathrm{Hg} \text { halo- } \\
\text { gen lamps, } 190-1000 \mathrm{~nm}\end{array}$ \\
\hline degasser & analytical 2-channel-online-degasser & analytical 2-channel-online-degasser \\
\hline
\end{tabular}


Table S22: HPLC configuration for preparative HPLC.

\begin{tabular}{l|l|l}
\hline & System D & System E \\
\hline series & Azura & Smartline \\
pumps & binary, HPG P 6.1L, 50 mL & binary, S-1800, 100 mL \\
pressure & 200-300 bar & 400 bar \\
autosampler & assistant ASM 2.1L with a feed pump P 2.1S & assistant 6000 with a feed pump S-100 \\
mixing chamber & integrated static mixing chamber, 200 $\mu \mathrm{L}$ & static, SmartMix 350 $\mu \mathrm{L}$ \\
detection type & MWL 2.1L, 190-700 nm & UV-detector S-2550, 190-600 nm \\
degasser & preparative 2-channel-online-degasser & preparative 2-channel-online-degasser \\
\hline
\end{tabular}

\subsubsection{Isomerization of Archazolid B}

Table S23: HPLC configuration for analysis and semi-preparative isolation of archazolid F.

\begin{tabular}{c|c|c}
\hline & analytical HPLC & semi-preparative HPLC \\
\hline system & A (Table S21) & E (Table S22) \\
column & Nucleodur $100-5$ Gravity C18 & Nucleodur $110-5$ Gravity C18 \\
particle diameter & $5 \mu \mathrm{m}$ & $5 \mu \mathrm{m}$ \\
pore size & $100 \AA$ & $110 \AA$ \\
column length & $250 \mathrm{~mm}$ & $250 \mathrm{~mm}$ \\
internal diameter & $4.0 \mathrm{~mm}$ & $16.0 \mathrm{~mm}$ \\
solvent & $\mathrm{MeOH} / \mathrm{H}_{2} \mathrm{O} 17: 3$ & $\mathrm{MeOH} / \mathrm{H}_{2} \mathrm{O} 85: 15$ \\
temperature & $25^{\circ} \mathrm{C}$ & $\mathrm{rt}$ \\
flow rate & $1.0 \mathrm{~mL} / \mathrm{min}$ & $16.0 \mathrm{~mL} / \mathrm{min}$ \\
\hline
\end{tabular}




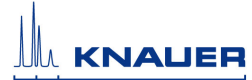

Andreas J. Schneider

Raum 5.067 / 5.068

Telefon: $73-5817$

Mail: a.schneider@uni-bonn.de

Rheinische Friedrich-Wilhelms-Universität Bonn

Kekulé-Institut für Organische Chemie und Biochemie

HPLC-Pool - hplcpool.uni-bonn.de

Messprotokoll SEMI-PRÄPARATIVE HPLC-Trennung

\begin{tabular}{|c|c|c|c|}
\hline Datum: 01. 06. 2015 & \multicolumn{2}{|c|}{ Name: Stephan Scheeff } & AK-Nr.: ME-033 \\
\hline \multicolumn{3}{|c|}{ Chiffre Substanz: SCHD12 } & Chiffre HPLC: ME-033-07 \\
\hline \multicolumn{4}{|c|}{ PROBENVORBEREITUNG } \\
\hline Menge: ca. $5 \mathrm{mg}$ & \multicolumn{2}{|c|}{ gelöst in $200 \mu \mathrm{l}$ Methanol } & Ultraschallbad? JA $\square$ NEIN X \\
\hline Filtriert? JA $\square$ NEIN X & \multicolumn{3}{|l|}{ Filterbezeichnung: } \\
\hline \multicolumn{4}{|c|}{ Anmerkungen: farblose Lösung } \\
\hline \multicolumn{4}{|c|}{ SYSTEMANGABEN } \\
\hline System TITAN $\mathrm{X}$ & System RHEA $\square$ & \multicolumn{2}{|c|}{ System TETHYS $\square$} \\
\hline Ri-Detektor $\square$ & \multicolumn{3}{|c|}{ UV X Wellenlängen: K1: $220 \mathrm{~nm}$ K2: $230 \mathrm{~nm}$ K3: $240 \mathrm{~nm}$ K4: --- nm } \\
\hline Eluenten & \multicolumn{2}{|l|}{ A: Wasser PureLab } & B: Methanol HPLCgrade \\
\hline Entgasung & \multicolumn{2}{|l|}{ Online-Degasser $\mathrm{X}$} & Ultraschallbad/Vakuum $\square$ \\
\hline
\end{tabular}

\begin{tabular}{|l|l|l|l|}
\hline LAUF 01 & Flußrate: $16,0 \mathrm{ml} / \mathrm{min}$ & Druck:220 bar & Injektion: $100 \mu \mathrm{l}$ (ca. 2,5 mg) \\
\hline
\end{tabular}

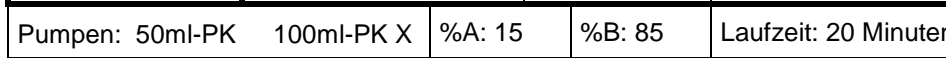

Isokratisch $X \quad$ Gradient $\square$ - Details:

Säule: MACHEREY-NAGEL Nucleodur Gravity C18, 5 mm, $250 \times 16,0$ mm + Vorsäulenkartusche

\begin{tabular}{l|l} 
Filename: ME-033-07-010615-01 & Anmerkungen: Trennung gut, analog analytischer Methode
\end{tabular}

\begin{tabular}{|l|l}
\hline Fraktionen: 2 Fraktionen aufgesammelt & Plot? JA $\square$ NEIN X
\end{tabular}

\begin{tabular}{|l|l|l|l}
\hline LAUF 02 & Flußrate: $16,0 \mathrm{ml} / \mathrm{min}$ & Druck:220 bar & Injektion: $100 \mu \mathrm{l}$ (ca. 2,5 mg)
\end{tabular}

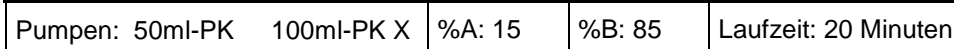

Isokratisch $X \quad$ Gradient $\square$ - Details:

Säule: MACHEREY-NAGEL Nucleodur Gravity C18, 5 m, 250 x 16,0 mm + Vorsäulenkartusche

\begin{tabular}{l|l} 
Filename: ME-033-07-010615-02 & Anmerkungen: reprod. Lauf 01
\end{tabular}

Fraktionen: 2 Fraktionen aufgesammelt

Plot? JA X NEIN

\begin{tabular}{|l|l|l|l}
\hline LAUF 03 & Flußrate: 16,0 ml/min & Druck:220 bar & Injektion: $400 \mu l$ - Restmengen!
\end{tabular}

\begin{tabular}{|c|c|c|c|c|c|}
\hline \multicolumn{2}{|c|}{ Pumpen: $50 \mathrm{ml}-\mathrm{PK} \square 100 \mathrm{ml}-\mathrm{PK} \mathrm{X}$} & $\%$ A: 15 & \%B: 85 & \multicolumn{2}{|c|}{ Laufzeit: 20 Minuten } \\
\hline Isokratisch X & \multicolumn{5}{|c|}{ Gradient $\square$ - Details: } \\
\hline \multicolumn{6}{|c|}{ Säule: MACHEREY-NAGEL Nucleodur Gravity C18P, 5 m, 250 x 16,0 mm + Vorsäulenkartusche } \\
\hline \multicolumn{2}{|c|}{ Filename: ME-033-07-010615-03 } & \multicolumn{4}{|c|}{ Anmerkungen: reprod. Lauf 01} \\
\hline \multicolumn{5}{|c|}{ Fraktionen: 2 Fraktionen aufgesammelt } & Plot? JA $\square$ NEIN X \\
\hline
\end{tabular}




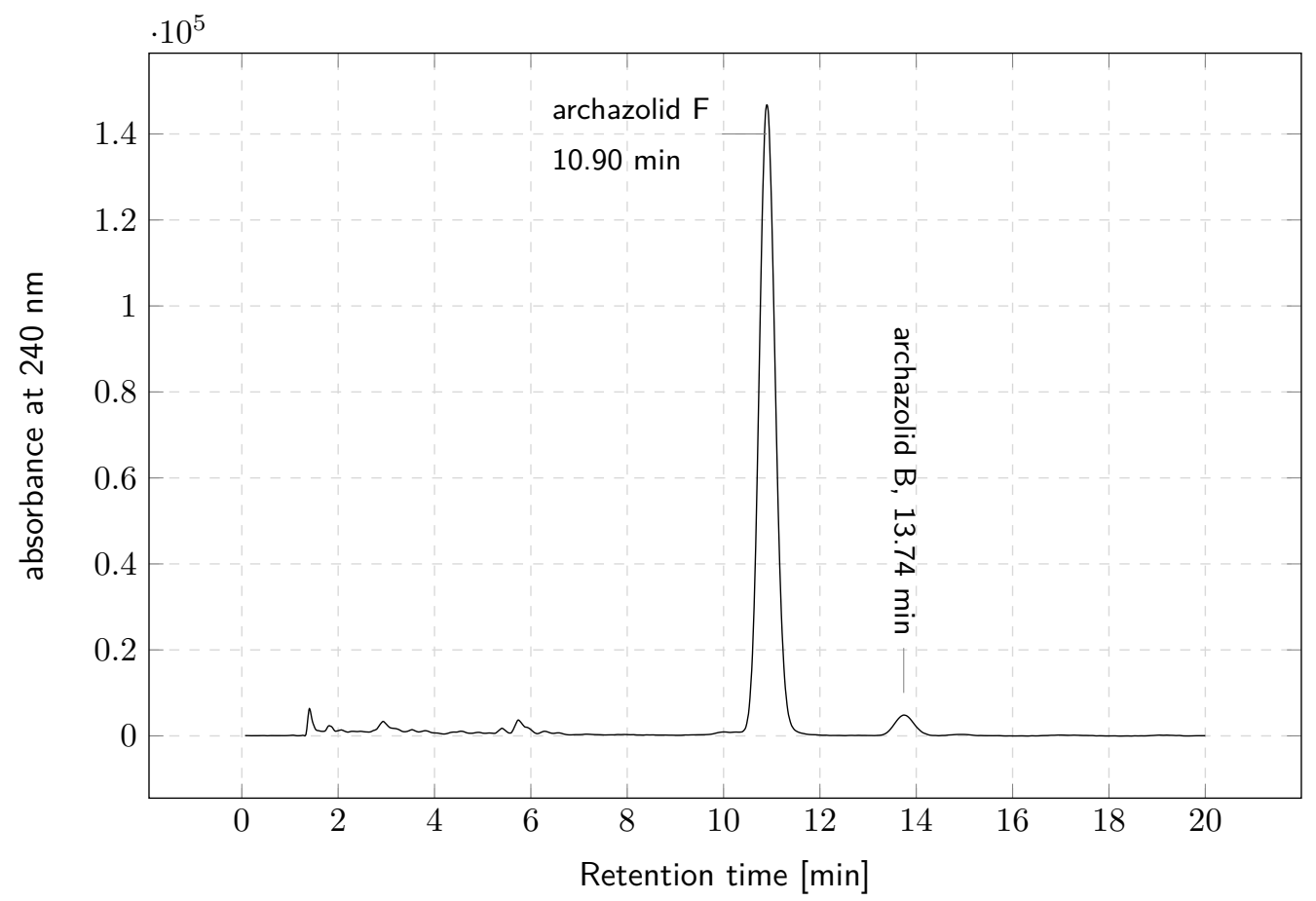

Figure S8. HPLC analysis of isomerization of archazolid B (2) to archazolid F (3) with DBU before semi-preparative HPLC seperation.

\subsubsection{Isomerization of Archazolid A}

Table S24: HPLC configuration for analysis and semi-preparative isolation of iso archazolid $\mathrm{A}_{1}$ and $\mathrm{A}_{2}$.

\begin{tabular}{c|c|c}
\hline & analytical HPLC & semi-preparative HPLC \\
\hline system & A (Table S21) & E (Table S22) \\
column & Nucleodur $100-5$ Gravity C18 & Nucleodur $110-5$ Gravity C18 \\
particle diameter & $5 \mu \mathrm{m}$ & $5 \mu \mathrm{m}$ \\
pore size & $100 \AA$ & $100 \AA$ \\
column length & $250 \mathrm{~mm}$ & $250 \mathrm{~mm}$ \\
internal diameter & $4.0 \mathrm{~mm}$ & $16.0 \mathrm{~mm}$ \\
\hline solvent & $\mathrm{ACN} / \mathrm{H}_{2} \mathrm{O} 9: 1$ & $\mathrm{ACN} / \mathrm{H}_{2} \mathrm{O} \mathrm{9:1}$ \\
temperature & $25^{\circ} \mathrm{C}$ & $\mathrm{rt}$ \\
flow rate & $1.0 \mathrm{~mL} / \mathrm{min}$ & $16.0 \mathrm{~mL} / \mathrm{min}$ \\
\hline
\end{tabular}




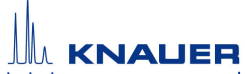

Andreas J. Schneider

Raum 5.067 / 5.068

Telefon: 73-5817

Mail: a.schneider@uni-bonn.de

Rheinische Friedrich-Wilhelms-Universität Bonn Kekulé-Institut für Organische Chemie und Biochemie HPLC-Pool - hplcpool.uni-bonn.de

Messprotokoll SEMI-PRÄPARATIVE HPLC-Trennung

\begin{tabular}{|l|l|l|}
\hline Datum: 06. 07. 2015 & Name: Stephan Scheeff & AK-Nr.: ME-033 \\
\hline Chiffre Substanz: SCHD25 & \multicolumn{1}{|c|}{ Chiffre HPLC: ME-033-13 } \\
\hline \multicolumn{3}{|c|}{ PROBENVORBEREITUNG } \\
\hline Menge: ca. $10 \mathrm{mg}$ & gelöst in 300 $\mu$ l Methanol & Ultraschallbad? JA $\square$ NEIN X \\
\hline Filtriert? JA $\square$ NEIN X & Filterbezeichnung: \\
\hline Anmerkungen: farblose Lösung - weißer, schmieriger Feststoff bleibt ungelöst. \\
\hline \multicolumn{3}{|c|}{ SYSTEMANGABEN } \\
\hline System TITAN X & System RHEA $\square$ & System TETHYS $\square$ \\
\hline Ri-Detektor $\square$ & UV X Wellenlängen: K1: 210 nm K2: 230 nm K3: $260 \mathrm{~nm} \mathrm{K4:280} \mathrm{nm}$ \\
\hline Eluenten & A: Acetonitril HPLCgrade & B: Wasser PureLab \\
\hline Entgasung & Online-Degasser X & Ultraschallbad/Vakuum $\square$ \\
\hline
\end{tabular}

\begin{tabular}{|l|l|l|l|}
\hline LAUF 01 & Flußrate: $16,0 \mathrm{ml} / \mathrm{min}$ & Druck:105 bar & Injektion: $100 \mu \mathrm{l}$ (ca. 3,33 mg)
\end{tabular}

\begin{tabular}{|l|l|l|l|l|}
\hline Pumpen: 50ml-PK $\square$ 100ml-PK X & $\% A: 90$ & $\% B: 10$ & Laufzeit: 20 Minuten \\
\hline
\end{tabular}

Isokratisch X $\quad$ Gradient $\square$ - Details:

Säule: MACHEREY-NAGEL Nucleodur Gravity C18, $5 \mu \mathrm{m}, 250 \times 16,0$ mm + Vorsäulenkartusche Filename: ME-033-15-060715-01 $\quad$\begin{tabular}{l}
$\begin{array}{l}\text { Anmerkungen: Trennung gut, etwas langsamer als analytischer } \\
\text { Methode }\end{array}$ \\
\hline
\end{tabular}

Fraktionen: 3 Fraktionen aufgesammelt Plot? JA $\square$ NEIN X

\begin{tabular}{|l|l|l|l}
\hline LAUF 02 - 04 & Flußrate: 16,0 ml/min & Druck:105 bar & Injektion: $100 \mu \mathrm{l}$ (ca. 3,33 mg)
\end{tabular}

\begin{tabular}{|c|c|c|}
\hline Pumpen: $50 \mathrm{ml}-\mathrm{PK} \square$ 100ml-PK X & $\% A: 90$ & $\% B: 10$ \\
\hline
\end{tabular}

Isokratisch $X \quad$ Gradient $\square$ - Details:

Säule: MACHEREY-NAGEL Nucleodur Gravity C18, $5 \mu \mathrm{m}, 250$ × 16,0 mm + Vorsäulenkartusche

Filename: ME-033-15-060715-02 bis ME-033-15-060715-04 $\quad$ Anmerkungen: reprod. Lauf 01

\begin{tabular}{|l|l}
\hline Fraktionen: 3 Fraktionen aufgesammelt & Plot? JA X NEIN
\end{tabular}

\section{Lauf 04 = Restmengen}

Lauf 03 ausgedruckt 


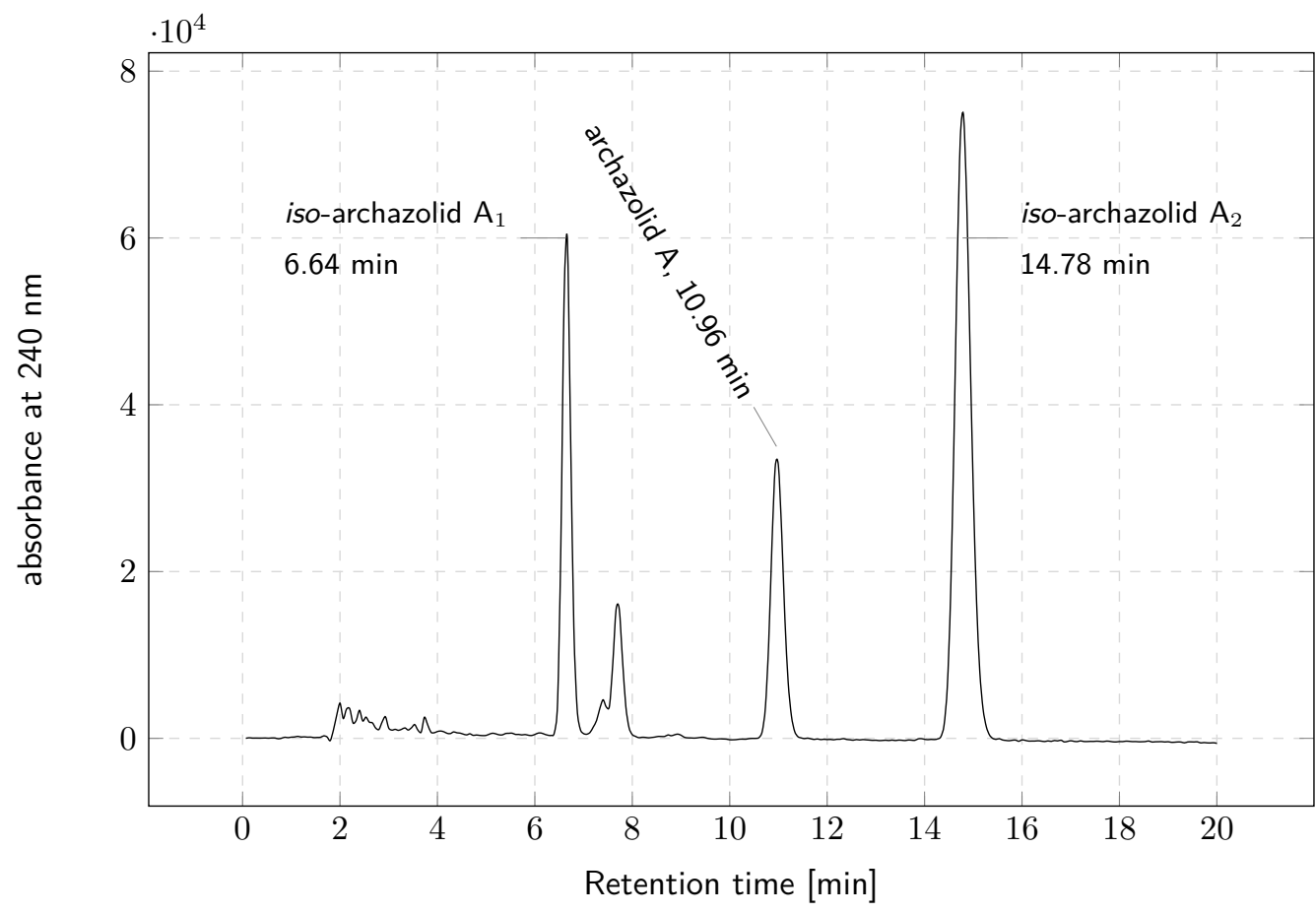

Figure S9. HPLC analysis of isomerization of archazolid A (1) to iso-archazolid $A_{1}(4)$ and iso-archazolid $A_{2}(5)$ before semi-preparative HPLC seperation.

\subsubsection{Synthesis of desmethyl-archazolid $F$}

Table S25: HPLC configuration for analysis and semi-preparative isolation of desmethyl archazolid F (6).

\begin{tabular}{c|c|c}
\hline & analytical HPLC & semi-preparative HPLC \\
\hline system & $\mathrm{C}($ Table S21) & E (Table S22) \\
column & Eurospher II 100-2 C18 & Eurospher II 100-5 C18 \\
particle diameter & $2 \mu \mathrm{m}$ & $5 \mu \mathrm{m}$ \\
pore size & $100 \AA$ & $100 \AA$ \\
column length & $100 \mathrm{~mm}$ & $250 \mathrm{~mm}$ \\
\hline internal diameter & $2.0 \mathrm{~mm}$ & $8.0 \mathrm{~mm}$ \\
\hline solvent & $\mathrm{ACN} / \mathrm{H}_{2} \mathrm{O} 4: 1$ & $\mathrm{ACN} / \mathrm{H}_{2} \mathrm{O} 4: 1$ \\
temperature & $25^{\circ} \mathrm{C}$ & $\mathrm{rt}$ \\
flow rate & $0.6 \mathrm{~mL} / \mathrm{min}$ & $5.0 \mathrm{~mL} / \mathrm{min}$ \\
\hline
\end{tabular}




\section{Jll KNAUER}

Andreas J. Schneider

Raum 5.067 / 5.068

Telefon: 73-5817

Mail: hplcpool@uni-bonn.de
Rheinische Friedrich-Wilhelms-Universität Bonn

Kekulé-Institut für Organische Chemie und Biochemie

HPLC-Pool - hplcpool.uni-bonn.de

Messprotokoll SEMI-PRÄPARATIVE HPLC-Trennung

\begin{tabular}{|c|c|c|c|}
\hline Datum: 28. 06. 2017 & \multicolumn{2}{|c|}{ Name: Stephan Scheeff } & AK-Nr.: ME-033 \\
\hline \multicolumn{2}{|c|}{ Chiffre Substanz: SCHA147F2 } & \multicolumn{2}{|c|}{ Chiffre HPLC: ME-033-47 } \\
\hline \multicolumn{4}{|c|}{ PROBENVORBEREITUNG } \\
\hline Menge: $3,4 \mathrm{mg}$ & \multicolumn{2}{|l|}{ gelöst in $200 \mu \mathrm{l} \mathrm{MeCN}$} & Ultraschallbad? JA $\square$ NEIN X \\
\hline Filtriert? JA $\square$ NEIN X & \multicolumn{3}{|l|}{ Filterbezeichnung: } \\
\hline \multicolumn{4}{|c|}{ Anmerkungen: farblose Flüssigkeit } \\
\hline \multicolumn{4}{|c|}{ SYSTEMANGABEN } \\
\hline System TITAN X & System RHEA $\square$ & \multicolumn{2}{|c|}{ System TETHYS $\square$} \\
\hline Ri-Detektor $\square$ & \multicolumn{3}{|c|}{ UV X Wellenlängen: K1: 230 nm K2: 260 nm K3: --- nm K4: --- nm } \\
\hline Eluenten & \multicolumn{2}{|l|}{ A: Acetonitril HPLCgrade } & B : Wasser PureLab \\
\hline Entgasung & \multicolumn{2}{|l|}{ Online-Degasser $\mathrm{X}$} & Ultraschallbad/Vakuum $\square$ \\
\hline
\end{tabular}

\begin{tabular}{|c|c|c|c|c|}
\hline LAUF 01 & \multicolumn{2}{|c|}{ Flußrate: $05,0 \mathrm{ml} / \mathrm{min}$} & Druck: 113 bar & Injektion: $100 \mu \mathrm{l}$ (ca. $1,7 \mathrm{mg})$ \\
\hline \multicolumn{2}{|c|}{ Pumpen: 50ml-PK $\square$ 100ml-PK X } & $\% A: 80$ & \%B: 20 & Laufzeit: 20 Minuten \\
\hline Isokratisch $\mathrm{X}$ & \multicolumn{4}{|l|}{ Gradient $\square$ - Details: } \\
\hline \multicolumn{5}{|c|}{ Säule: KNAUER Eurospher II 100-5 C18, 5 m, 250 x 8,0 mm mit Vorsäulen 30 x 8,0 mm } \\
\hline \multicolumn{2}{|c|}{ Filename: ME-033-47-280617-01 } & \multicolumn{3}{|c|}{ Anmerkungen: Trennung gut } \\
\hline \multicolumn{4}{|c|}{ Fraktionen: 1 Fraktion aufgesammelt } & Plot? JA $\square$ NEIN X \\
\hline LAUF 02 & \multicolumn{2}{|c|}{ Flußrate: $05,0 \mathrm{ml} / \mathrm{min}$} & Druck: 113 bar & Injektion: $100 \mu \mathrm{l}$ (ca. 1,7 mg) \\
\hline \multicolumn{2}{|c|}{ Pumpen: $50 \mathrm{ml}-\mathrm{PK} \square$ 100ml-PKX } & $\% A: 80$ & $\% B: 20$ & Laufzeit: 20 Minuten \\
\hline Isokratisch X & \multicolumn{4}{|l|}{ Gradient $\square$ - Details: } \\
\hline \multicolumn{5}{|c|}{ Säule: KNAUER Eurospher II 100-5 C18, 5 m, 250 x 8,0 mm mit Vorsäulen 30 x 8,0 mm } \\
\hline \multicolumn{2}{|c|}{ Filename: ME-033-47-280617-02 } & \multicolumn{3}{|c|}{ Anmerkungen: reprod. Lauf 01} \\
\hline \multicolumn{4}{|c|}{ Fraktionen: 1 Fraktion aufgesammelt } & Plot? JA X NEIN $\square$ \\
\hline LAUF 03 & \multicolumn{2}{|c|}{ Flußrate: $05,0 \mathrm{ml} / \mathrm{min}$} & Druck: 113 bar & Injektion: Restmengen \\
\hline \multicolumn{2}{|c|}{ Pumpen: 50ml-PK $\square$ 100ml-PKX } & $\% A: 80$ & $\%$ B: 20 & Laufzeit: 20 Minuten \\
\hline Isokratisch X & \multicolumn{4}{|l|}{ Gradient $\square$ - Details: } \\
\hline \multicolumn{5}{|c|}{ 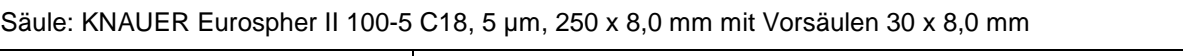 } \\
\hline \multicolumn{2}{|c|}{ Filename: ME-033-47-280617-03 } & \multicolumn{3}{|c|}{ Anmerkungen: Trennung gut } \\
\hline \multicolumn{4}{|c|}{ Fraktionen: 1 Fraktion aufgesammelt } & Plot? JA $\square$ NEIN X \\
\hline
\end{tabular}




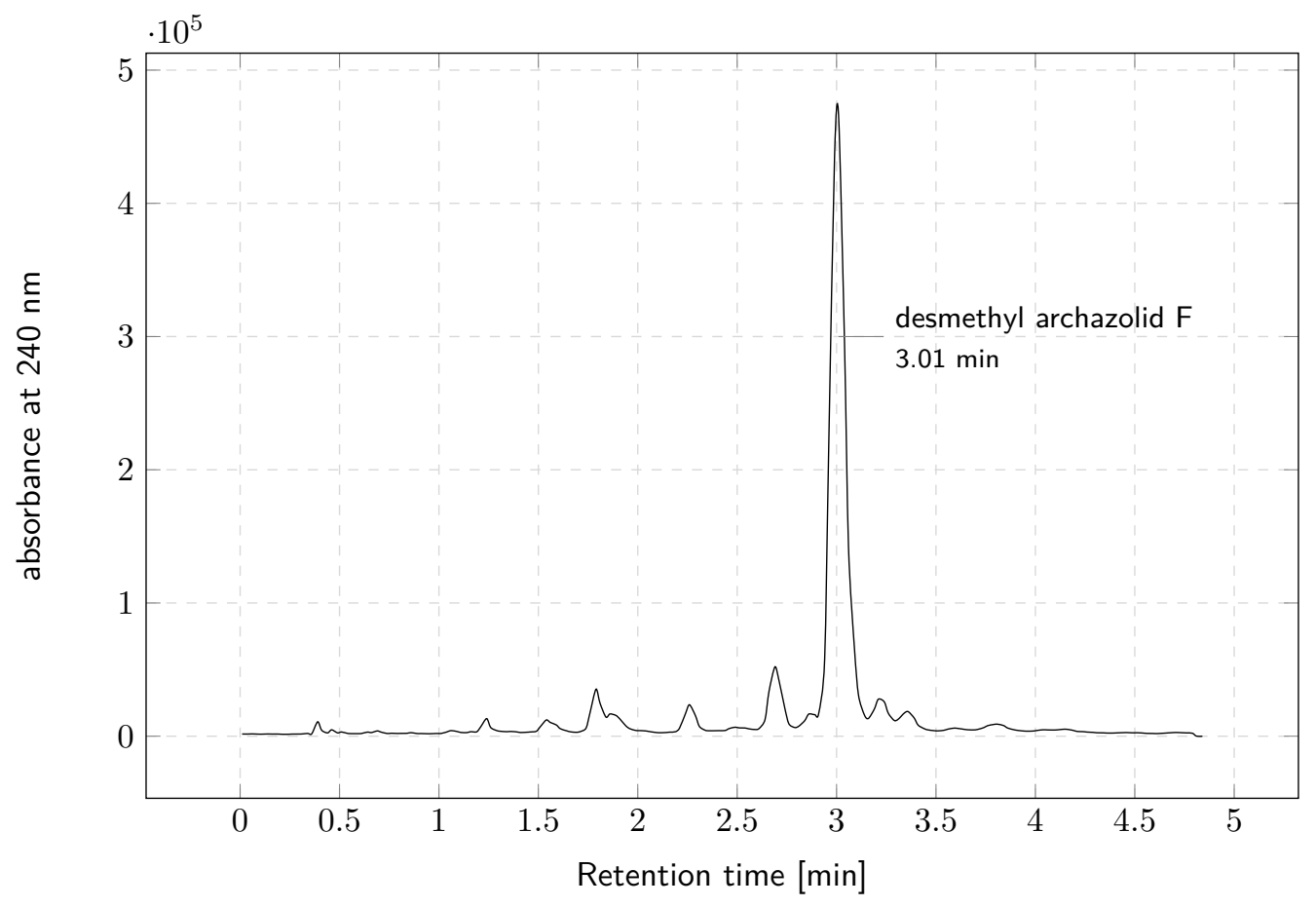

Figure S10. HPLC analysis after RCM and deprotection for the synthesis of desmethyl archazolid F (6) before semi-preparative HPLC seperation.

\subsubsection{Synthesis of archazolog}

Table S26: HPLC configuration for analysis and semi-preparative isolation of archazolog (7).

\begin{tabular}{c|c|c}
\hline & analytical HPLC & semi-preparative HPLC \\
\hline system & $\mathrm{C}($ Table S21) & E (Table S22) \\
column & Eurospher II 100-2 C18 & Eurospher II 100-5 C18 \\
particle diameter & $2 \mu \mathrm{m}$ & $5 \mu \mathrm{m}$ \\
pore size & $100 \AA$ & $100 \AA$ \\
column length & $100 \mathrm{~mm}$ & $250 \mathrm{~mm}$ \\
internal diameter & $2.0 \mathrm{~mm}$ & $8.0 \mathrm{~mm}$ \\
\hline solvent & $\mathrm{ACN} / \mathrm{H}_{2} \mathrm{O} 17: 3$ & $\mathrm{ACN} / \mathrm{H}_{2} \mathrm{O} 85: 15$ \\
temperature & $25^{\circ} \mathrm{C}$ & $\mathrm{rt}$ \\
flow rate & $0.6 \mathrm{~mL} / \mathrm{min}$ & $5.0 \mathrm{~mL} / \mathrm{min}$ \\
\hline
\end{tabular}




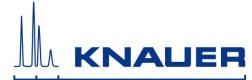

Andreas J. Schneider

Raum 5.067 / 5.068

Telefon: 73-5817

Mail: hplcpool@uni-bonn.de

Rheinische Friedrich-Wilhelms-Universität Bonn

Kekulé-Institut für Organische Chemie und Biochemie

HPLC-Pool - hplcpool.uni-bonn.de

Messprotokoll SEMI-PRÄPARATIVE HPLC-Trennung

\begin{tabular}{|c|c|c|c|}
\hline Datum: 21. 06. 2017 & \multicolumn{2}{|c|}{ Name: Stephan Scheeff } & AK-Nr.: ME-033 \\
\hline \multicolumn{2}{|c|}{ Chiffre Substanz: SCHA136 } & \multicolumn{2}{|c|}{ Chiffre HPLC: ME-033-45 } \\
\hline \multicolumn{4}{|c|}{ PROBENVORBEREITUNG } \\
\hline Menge: $9,6 \mathrm{mg}$ & \multicolumn{2}{|l|}{ gelöst in $200 \mu \mathrm{l} \mathrm{MeCN}$} & Ultraschallbad? JA $\square$ NEIN X \\
\hline Filtriert? JA $\square$ NEIN X & \multicolumn{3}{|l|}{ Filterbezeichnung: } \\
\hline \multicolumn{4}{|c|}{ Anmerkungen: farblose Flüssigkeit } \\
\hline \multicolumn{4}{|c|}{ SYSTEMANGABEN } \\
\hline System TITAN X & System RHEA $\square$ & \multicolumn{2}{|c|}{ System TETHYS $\square$} \\
\hline Ri-Detektor $\square$ & \multicolumn{3}{|c|}{ UV X Wellenlängen: K1: $230 \mathrm{~nm}$ K2: $260 \mathrm{~nm}$ K3: --- nm K4:--- nm } \\
\hline Eluenten & \multicolumn{2}{|l|}{ A: Acetonitril HPLCgrade } & B : Wasser PureLab \\
\hline Entgasung & \multicolumn{2}{|l|}{ Online-Degasser $\mathrm{X}$} & Ultraschallbad/Vakuum $\square$ \\
\hline
\end{tabular}

\begin{tabular}{|c|c|c|c|c|}
\hline LAUF 01 & \multicolumn{2}{|c|}{ Flußrate: $05,0 \mathrm{ml} / \mathrm{min}$} & Druck: 102 bar & Injektion: $100 \mu \mathrm{l}$ (ca. 4,8 mg) \\
\hline \multicolumn{2}{|c|}{ Pumpen: $50 \mathrm{ml}-\mathrm{PK} \square$ 100ml-PK X } & $\% A: 85$ & $\% \mathrm{~B}: 15$ & Laufzeit: 20 Minuten \\
\hline Isokratisch $\mathrm{X}$ & \multicolumn{4}{|c|}{ Gradient $\square$ - Details: } \\
\hline \multicolumn{5}{|c|}{ Säule: KNAUER Eurospher II 100-5 C18, $5 \mu \mathrm{m}, 250$ x 8,0 mm mit Vorsäulen 30 x 8,0 mm } \\
\hline \multicolumn{2}{|c|}{ Filename: ME-033-45-210617-01 } & \multicolumn{3}{|c|}{ Anmerkungen: Trennung gut } \\
\hline \multicolumn{4}{|c|}{ Fraktionen: 2 Fraktionen aufgesammelt } & Plot? JA $\square$ NEIN X \\
\hline LAUF 02 & \multicolumn{2}{|c|}{ Flußrate: $05,0 \mathrm{ml} / \mathrm{min}$} & Druck: 102 bar & Injektion: $100 \mu \mathrm{l}$ (ca. $4,8 \mathrm{mg}$ ) \\
\hline \multicolumn{2}{|c|}{ Pumpen: $50 \mathrm{ml}-\mathrm{PK} \square 100 \mathrm{ml}-\mathrm{PK} \mathrm{X}$} & \%A: 85 & $\% \mathrm{~B}: 15$ & Laufzeit: 20 Minuten \\
\hline Isokratisch X & \multicolumn{4}{|l|}{ Gradient $\square$ - Details: } \\
\hline \multicolumn{5}{|c|}{ Säule: KNAUER Eurospher II 100-5 C18, 5 m, 250 x 8,0 mm mit Vorsäulen 30 x 8,0 mm } \\
\hline \multicolumn{2}{|c|}{ Filename: ME-033-45-210617-02 } & \multicolumn{3}{|c|}{ Anmerkungen: reprod. Lauf 01 - Restmengen } \\
\hline \multicolumn{4}{|c|}{ Fraktionen: 2 Fraktionen aufgesammelt } & Plot? JA X NEIN \\
\hline LAUF 03 & \multicolumn{2}{|c|}{ Flußrate: $05,0 \mathrm{ml} / \mathrm{min}$} & Druck: 102 bar & Injektion: Restmengen \\
\hline \multicolumn{2}{|c|}{ Pumpen: 50ml-PK $\square$ 100ml-PKX } & $\%$ A: 85 & $\%$ B: 15 & Laufzeit: 20 Minuten \\
\hline Isokratisch X & \multicolumn{3}{|l|}{ Gradient $\square$ - Details: } & \\
\hline \multicolumn{5}{|c|}{ Säule: KNAUER Eurospher II 100-5 C18, 5 m, 250 x 8,0 mm mit Vorsäulen 30 x 8,0 mm } \\
\hline \multicolumn{2}{|c|}{ Filename: ME-033-45-210617-03 } & \multicolumn{3}{|c|}{ Anmerkungen: reprod. Lauf 01 - Restmengen } \\
\hline \multicolumn{4}{|c|}{ Fraktionen: 2 Fraktionen aufgesammelt } & Plot? JA $\square$ NEIN X \\
\hline
\end{tabular}

Lauf 03 = Restmengen 


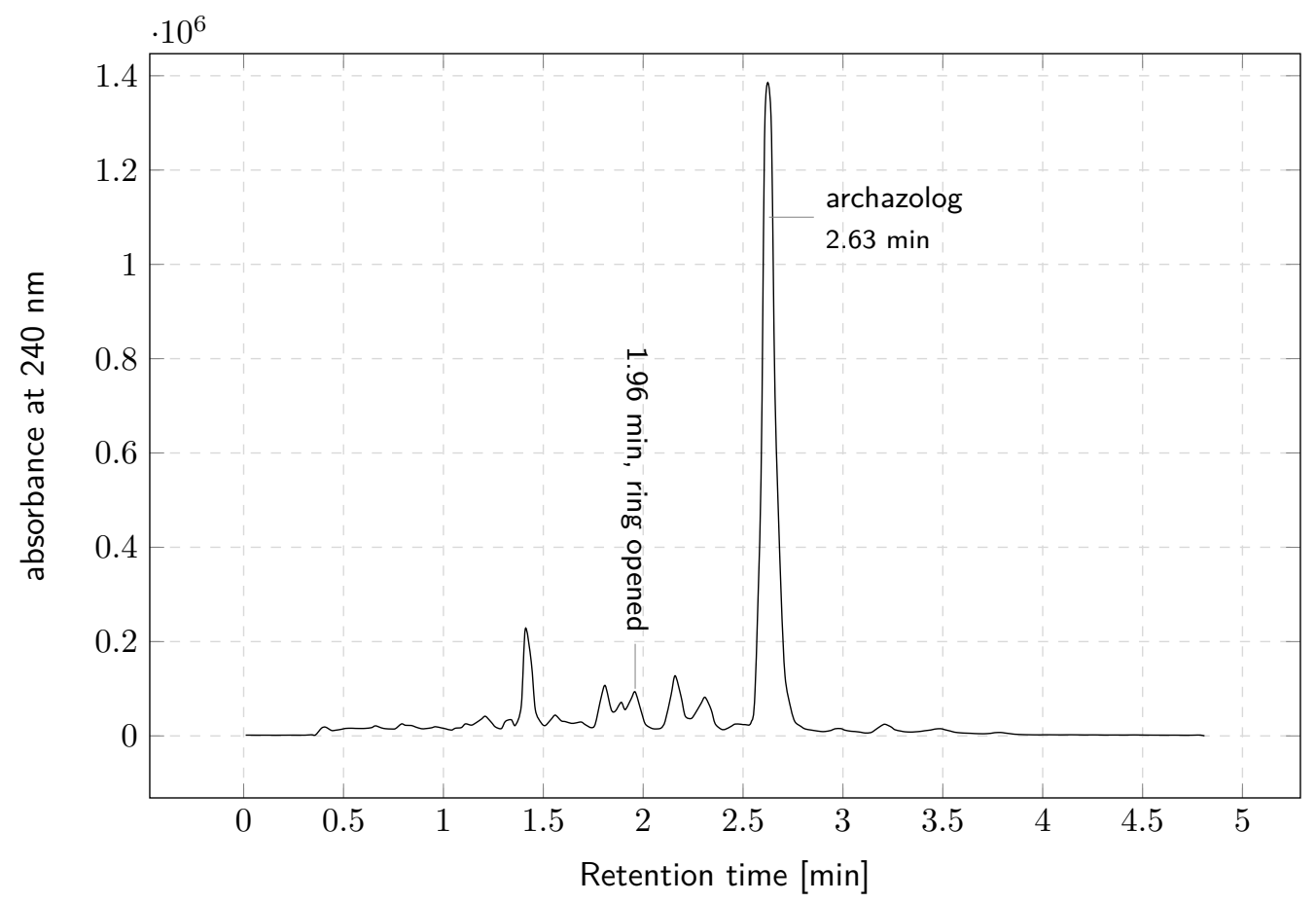

Figure S11. HPLC analysis after RCM and deprotection for the synthesis of archazolog (7) before semi-preparative HPLC seperation. 


\subsection{Proof of purity}

- see next page - 
Sequence Name:

Data file:

Sample name:

Instrument:

Inj. volume:

Acq. method:

Processing method:
2019-09-26_Menche

Menche 2019-09-26 08-20-19+02-00.dx Project Name:

ArchazolidA

Single Quad

5.000

0_wasser-acn_standard_100-1000_AC.amx

MS_standard_plot.pmx
Single Quad

SYSTEM

Operator :

Acquired on:

2019-09-26 08:22:02+02:00

Location:

$\mathrm{P} 1-\mathrm{A} 1$
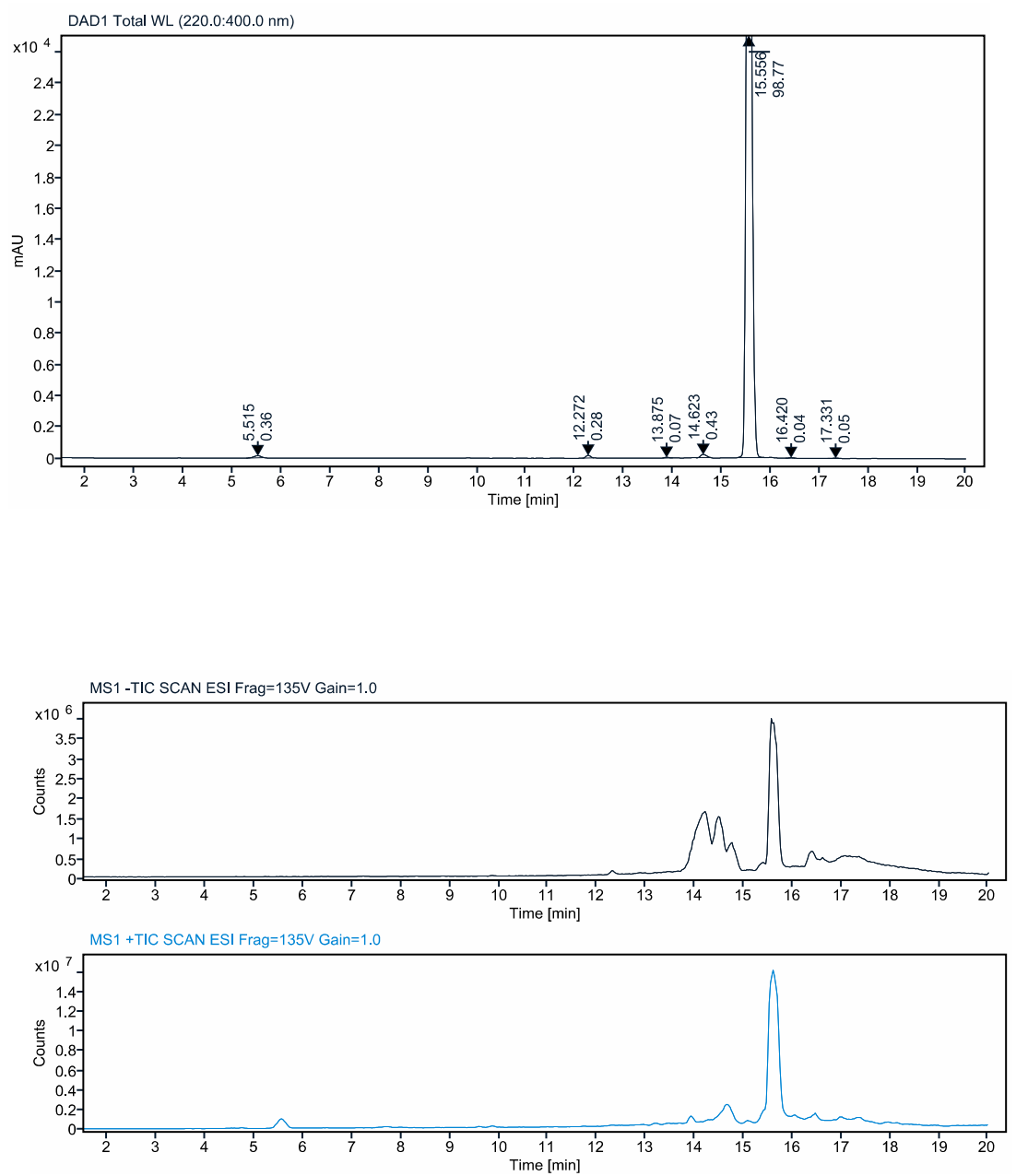
Signal: $\quad$ DAD1 Total WL $(220.0: 400.0 \mathrm{~nm})$

RT [min] Type Width [min] Colo

$\begin{array}{rl}5.515 \mathrm{MM} \mathrm{m} & 0.1619 \\ 12.272 \mathrm{MM} \mathrm{m} & 0.1028 \\ 13.875 \mathrm{MM} \mathrm{m} & 0.1017 \\ 14.623 \mathrm{MM} \mathrm{m} & 0.1328 \\ 15.556 \mathrm{MM} \mathrm{m} & 0.1257 \\ 16.420 \mathrm{MM} \mathrm{m} & 0.0945 \\ 17.331 \mathrm{MM} \mathrm{m} & 0.1066\end{array}$

(7.331

\section{Area}

1367.2681

1056.2762

277.6020

1657.7362

377097.7780

153.0760

174.0301

Sum 381783.7665

\section{Height}

135.3530

169.3159

45.1396

204.1126

47950.9869

27.6845

25.8252

Signal: $\quad$ MS1 +TIC SCAN ESI Frag $=135 \mathrm{~V}$ Gain $=1.0$

RT [min] Type Width [min] Colo

Area

15.608 BV

0.7409

$\begin{array}{rrr} & 220959072 . & 15407743.9 \\ & 5333 & 295 \\ \text { Sum } & 220959072 . & \end{array}$

5333

Signal: $\quad$ MS1 - TIC SCAN ESI Frag $=135 \mathrm{~V}$ Gain $=1.0$

RT [min] Type Width [min] Colo

Area

15.576 MM m

0.1503

$\begin{array}{rr}42195311.2 & 3682079.24 \\ 685 & 98\end{array}$

Sum 42195311.2

\section{Area\% Peak MS Base}

0.3581

0.2767

0.0727

0.4342

98.7726

0.0401

0.0456

Area\% Peak MS Base

100.0000

Peak m/z

Area\% Peak MS Base

100.0000
Peak $\mathrm{m} / \mathrm{z}$ 

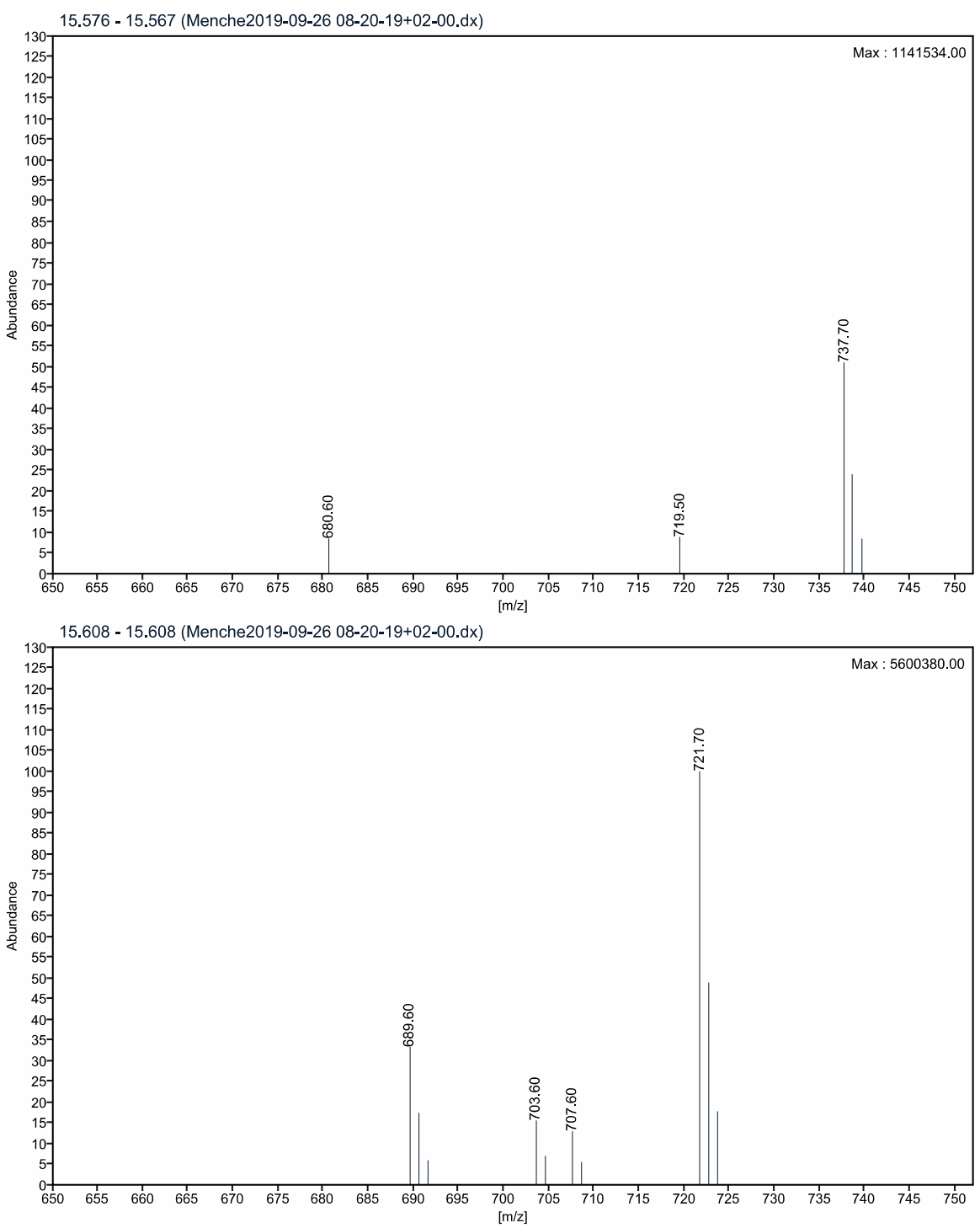

Figure S12. LC-MS spectrum of archazolid A. The purity of archazolid A is $98.8 \%$ (retention time: $15.6 \mathrm{~min}$ corresponds to the desired compound) 
Sequence Name:

Data file:

Sample name:

Instrument:

Inj. volume:

Acq. method:

Processing method:
2019-09-26_Menche

Menche2019-09-26 08-47-03+02-00.dx Project Name:

ArchazolidB

Single Quad

10.000

Operator :

Acquired on:

Location:

0_wasser-acn_standard_100-1000_AC-long.amx

MS_standard_plot.pmx
Single Quad

SYSTEM

2019-09-26 08:48:02+02:00

$\mathrm{P} 1-\mathrm{A} 2$
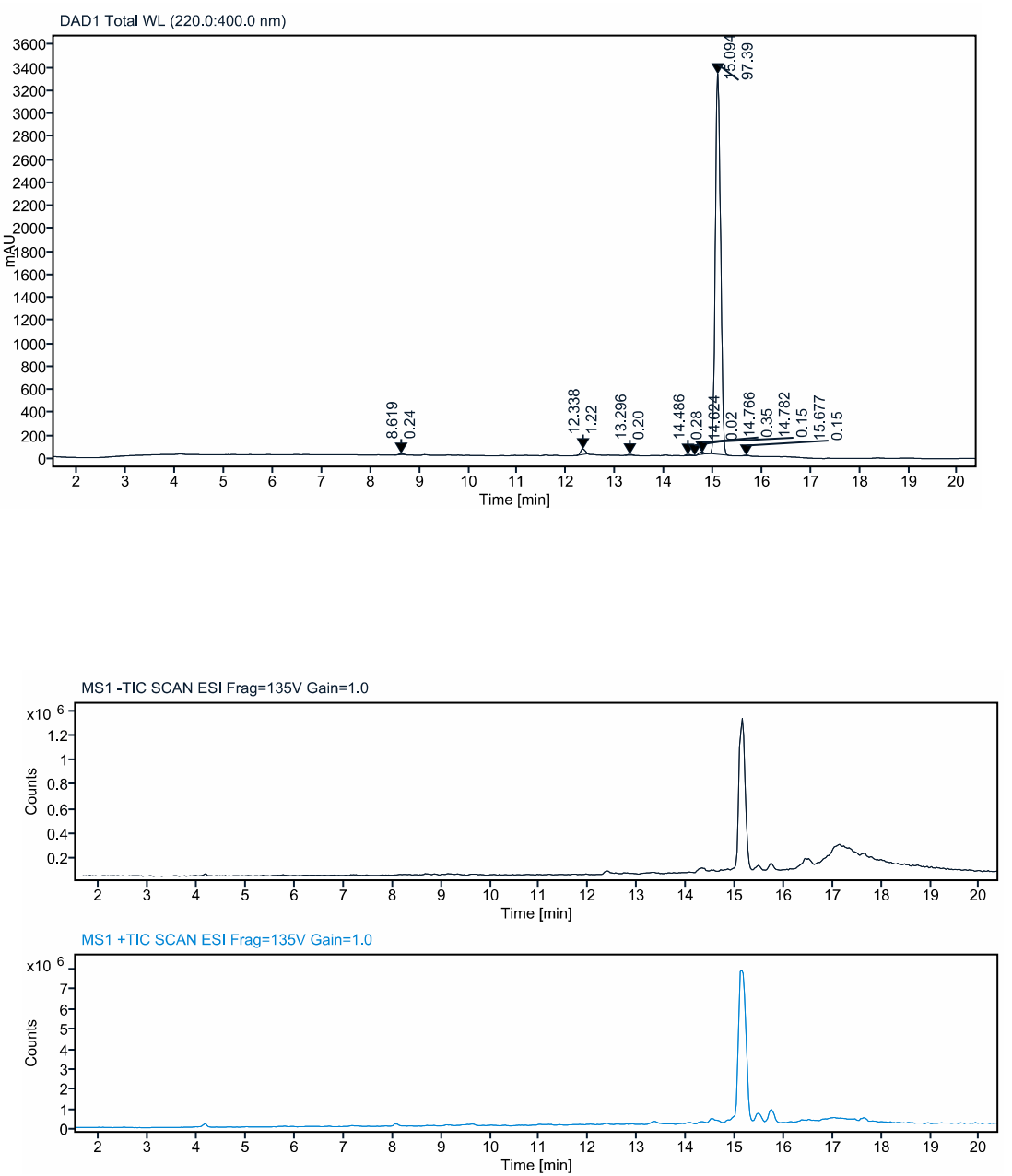
Signal: $\quad$ DAD1 Total WL $(220.0: 400.0 \mathrm{~nm})$

RT [min] Type Width [min] Colo

\begin{tabular}{|c|c|c|}
\hline $8.619 \mathrm{MM} \mathrm{m}$ & 0.0920 & 61.0004 \\
\hline $12.338 \mathrm{MM} \mathrm{m}$ & 0.1066 & 310.4417 \\
\hline 13.296 MM m & 0.1077 & 50.0854 \\
\hline 14.486 MM m & 0.1214 & 72.4122 \\
\hline $14.624 \mathrm{MM} \mathrm{t}$ & 0.0693 & 5.4397 \\
\hline 14.766 MM m & 0.0788 & 90.3475 \\
\hline $14.782 \mathrm{MM} \mathrm{m}$ & 0.0366 & 38.0141 \\
\hline $15.094 \mathrm{MM} \mathrm{m}$ & 0.1195 & 24822.8548 \\
\hline \multirow[t]{2}{*}{$15.677 \mathrm{MM} \mathrm{m}$} & 0.1048 & 37.4170 \\
\hline & & 25488.0128 \\
\hline
\end{tabular}

Signal: $\quad$ MS1 +TIC SCAN ESI Frag=135V Gain=1.0

RT [min] Type Width [min] ColO
$15.133 \mathrm{BV}$
0.5884
\begin{tabular}{rr}
051 & 7601050.45 \\
\hline
\end{tabular}

Sum 81190845.2

051

Signal: $\quad$ MS1 - TIC SCAN ESI Frag $=135 \mathrm{~V}$ Gain=1.0

RT [min] Type Width [min] Colo

Area

15.150 MM m

0.1324

$\begin{array}{rr}10197854.3 & 1198261.58 \\ 715 & 55\end{array}$

Sum 10197854.3

Height

Area $\%$

\section{Height Area\% Peak MS Base}

$10.7777 \quad 0.2393$

$47.2721 \quad 1.2180$

$8.1459 \quad 0.1965$

$10.8875 \quad 0.2841$

$1.3087 \quad 0.0213$

$19.0990 \quad 0.3545$

$17.3275 \quad 0.1491$

$3308.9846 \quad 97.3903$

0.1468

100.0000

Peak MS Base

Peak m/z

Area\% Peak MS Base

Peak m/z

100.0000 

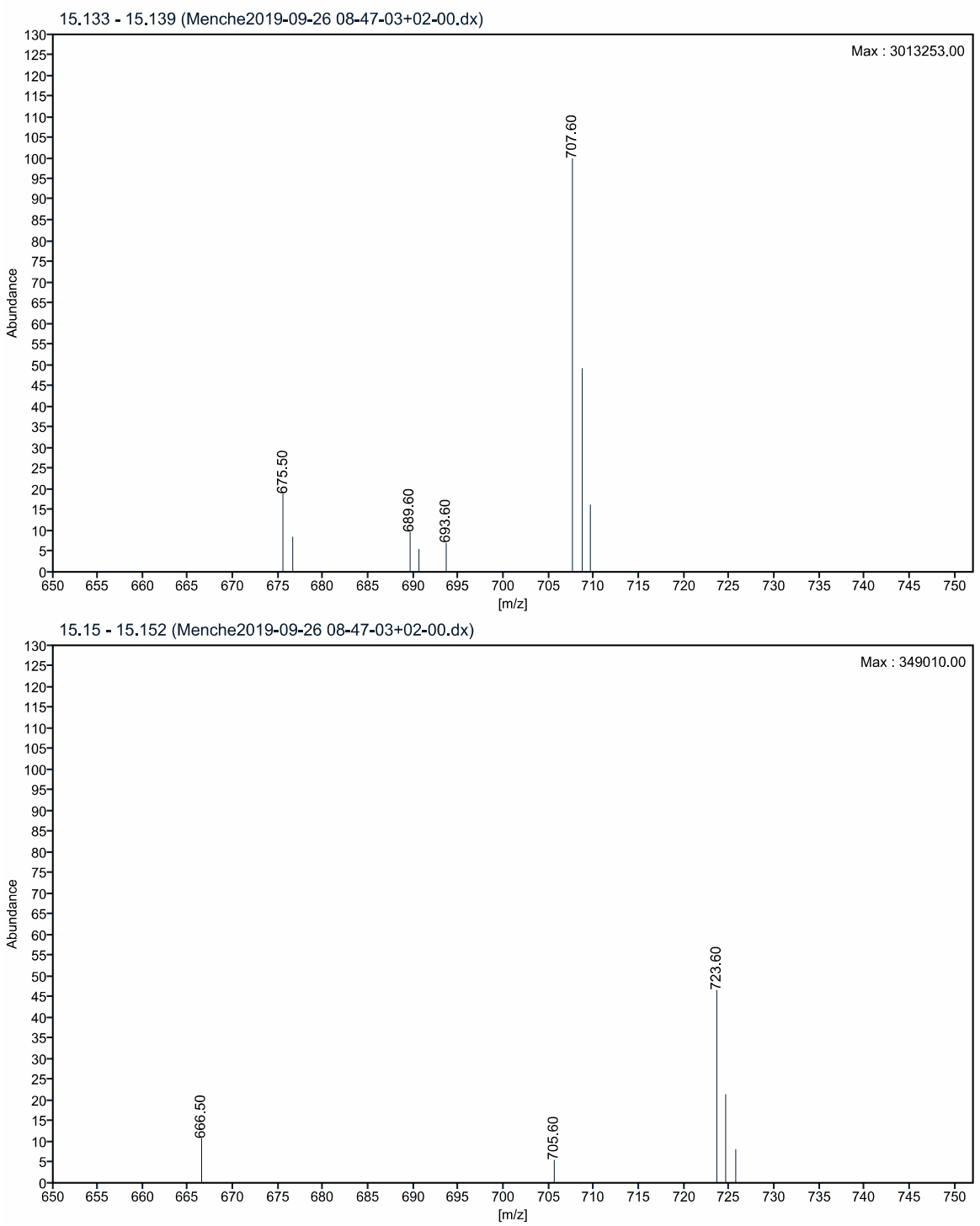

Figure S13. LC-MS spectrum of archazolid B. The purity of archazolid B is $97.4 \%$ (retention time: 15.1 min corresponds to the desired compound) 
Sequence Name:

Data file:

Sample name:

Instrument:

Inj. volume:

Acq. method:

Processing method:
2019-09-26_Menche

Menche2019-09-26 09-18-03+02-00.dx Project Name:

ArchazolidF

Single Quad

5.000

0 wasser-acn standard 100-1000 AC-

MS_standard_plot.pmx
Single Quad

SYSTEM

2019-09-26 09:19:01+02:00

P1-A3
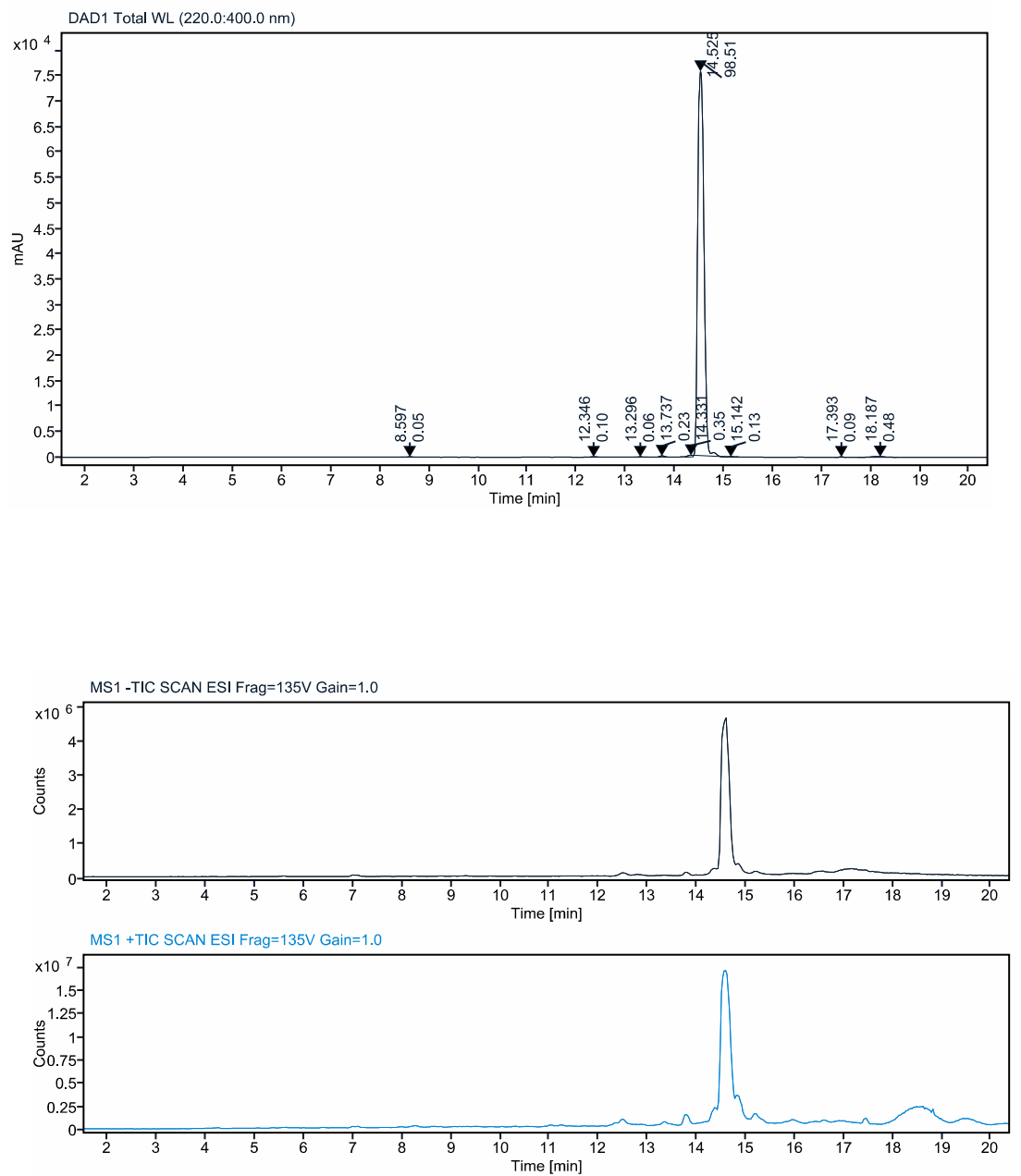
Signal: $\quad$ DAD1 Total WL $(220.0: 400.0 \mathrm{~nm})$

RT [min] Type Width [min] Colo

$\begin{array}{rl}\text { 8.597 MM m } & 0.1163 \\ 12.346 \mathrm{MM} \mathrm{m} & 0.1334 \\ 13.296 \mathrm{MM} \mathrm{m} & 0.1059 \\ 13.737 \mathrm{MM} \mathrm{m} & 0.1033 \\ 14.331 \mathrm{MM} \mathrm{m} & 0.1202 \\ 14.525 \mathrm{MM} \mathrm{m} & 0.1439 \\ 15.142 \mathrm{MM} \mathrm{m} & 0.1399 \\ 17.393 \mathrm{MM} \mathrm{m} & 0.0813 \\ 18.187 \mathrm{MM} \mathrm{m} & 0.2632\end{array}$

18.187

\section{Area}

350.6613

637.4144

380.9070

1546.0110

2362.8868

660661.6797

893.9875

585.3713

3251.8272

Sum 670670.7462 \begin{tabular}{r} 
Area\% \\
\hline 0.0523 \\
0.0950 \\
0.0568 \\
0.2305 \\
0.3523 \\
98.5076 \\
0.1333 \\
0.0873 \\
0.4849
\end{tabular}

Signal: $\quad$ MS1 +TIC SCAN ESI Frag $=135 \mathrm{~V}$ Gain $=1.0$

RT [min] Type Width [min] Colo

Area

14.575 MM m

0.1991

Height

Area\%

100.0000

\section{Peak MS Base}

Peak m/z

Sum 176212959

655

Signal: $\quad$ MS1 - TIC SCAN ESI Frag $=135 \mathrm{~V}$ Gain=1.0

RT [min] Type Width [min] Colo

Area

$14.589 \mathrm{MV} \mathrm{m}$

0.1729

$51028154.8 \quad 4526421.07$

676

Sum $\quad 51028154.8$

Peak MS Base
Peak m/z

$\left(\frac{10}{20}\right.$ 

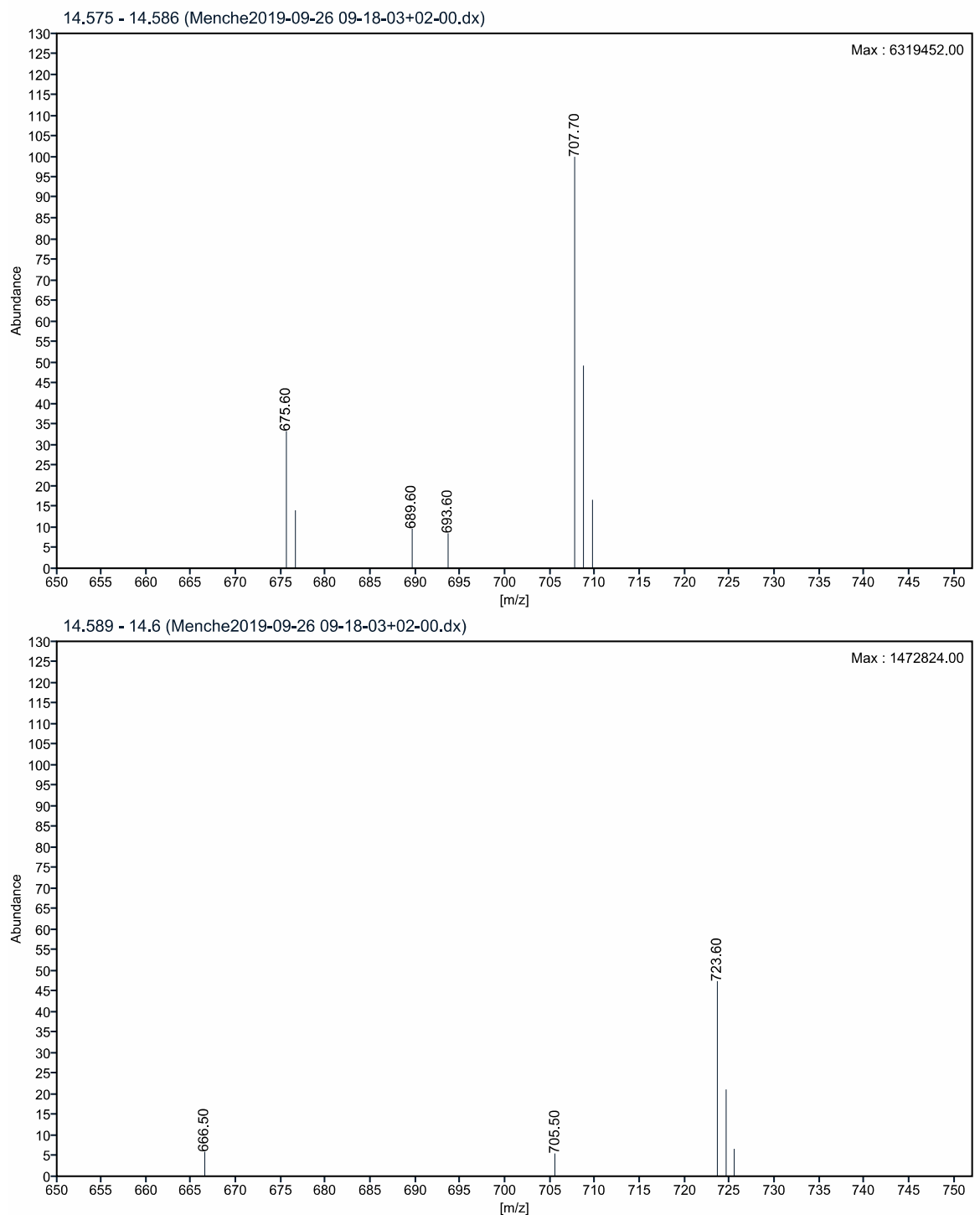

Figure S14. LC-MS spectrum of archazolid F. The purity of archazolid F is $98.5 \%$ (retention time: 14.5 min corresponds to the desired compound) 
Sequence Name:

Data file:

Sample name:

Instrument:

Inj. volume:

Acq. method:

Processing method:
2019-09-26_Menche

Menche2019-09-26 09-49-03+02-00.dx Project Name:

iso-ArchazolidA1

Single Quad

5.000

Operator :

Acquired on:

Location:

0_wasser-acn_standard_100-1000_AC-long.amx

MS_standard_plot.pmx
Single Quad

SYSTEM

2019-09-26 09:49:58+02:00

P1-A4
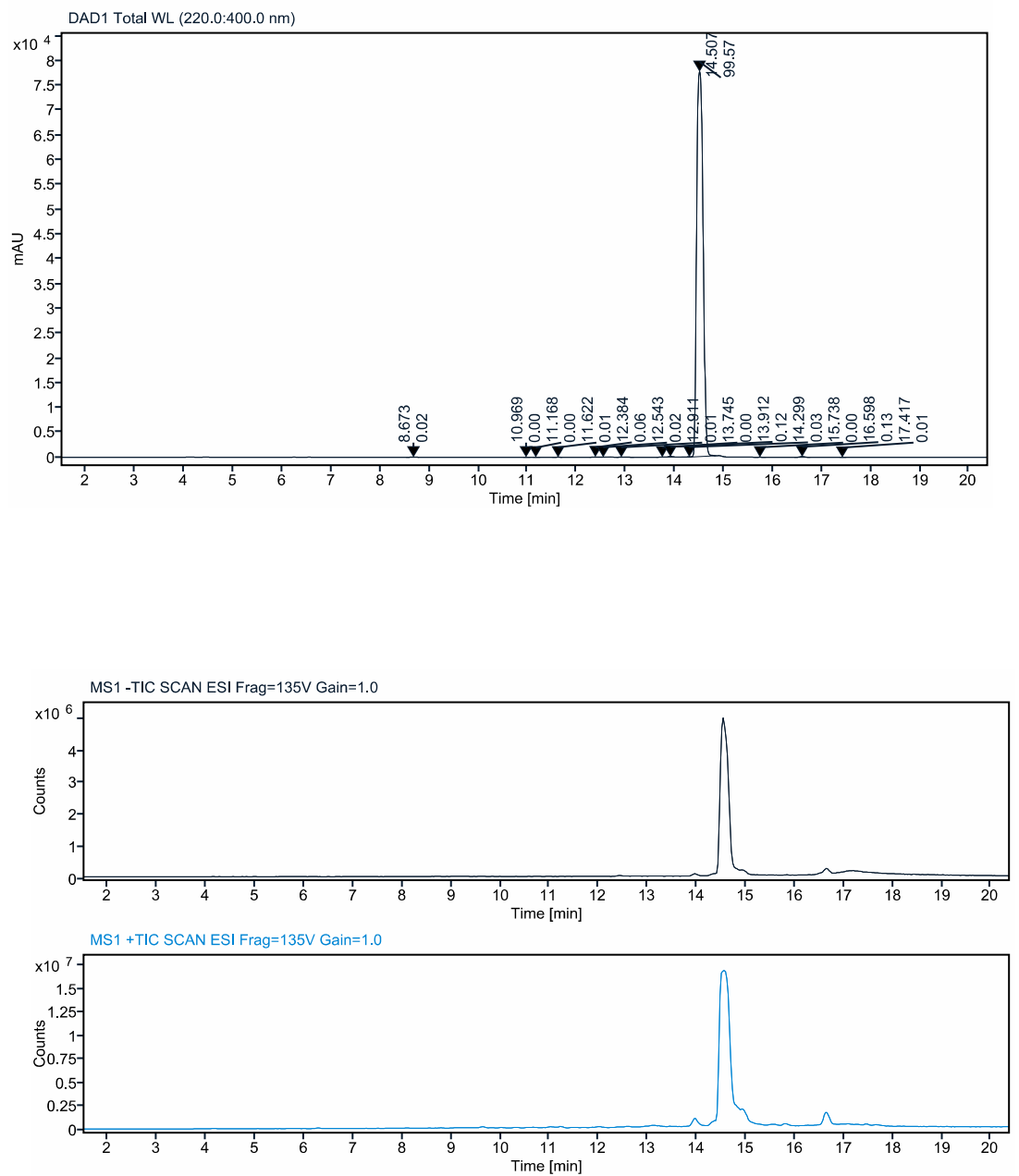
Signal: $\quad$ DAD1 Total WL $(220.0: 400.0 \mathrm{~nm})$

16.598 MM m
RT [min] Type Width [min] Colo

$8.673 \mathrm{MM} \mathrm{m}$

$10.969 \mathrm{MM} \mathrm{m}$

$11.168 \mathrm{MM} \mathrm{m}$

$11.622 \mathrm{MM} \mathrm{m}$

$12.384 \mathrm{MM} \mathrm{m}$

$12.543 \mathrm{MM} \mathrm{m}$

$12.911 \mathrm{MM} \mathrm{m}$

$13.745 \mathrm{MM} \mathrm{m}$

$13.912 \mathrm{MM} \mathrm{m}$

14.299 MM m

$14.507 \mathrm{MB} m$

$15.738 \mathrm{MM} \mathrm{m}$

$17.417 \mathrm{MM} \mathrm{m}$

\subsection{7}

\subsection{9}

0.0830

0.0929

0.1232

0.1011

0.1924

0.0318

0.1106

0.0836

0.1477

0.1124

0.1160

0.0740

\section{Area}

148.8173

16.7045

15.2802

62.3818

447.3512

163.0549

61.6974

4.7615

869.2643

246.4763

704539.0896

34.1067

945.7324

39.7796

Sum 707594.4977

\section{Height}

24.5609

3.5481

3.0678

11.2098

57.2376

25.3224

4.2739

2.4965

128.9575

49.1642

77754.1303

4.9516

131.3998

8.9194

Signal: $\quad$ MS1 +TIC SCAN ESI Frag=135V Gain=1.0

RT [min] Type Width [min] Colo

14.557 BM m

0.2420

$\begin{array}{rr}\text { Area } & \text { Height } \\ & \\ 242572891 . & 16109205.9 \\ 9026 & 754\end{array}$

Sum 242572891 .

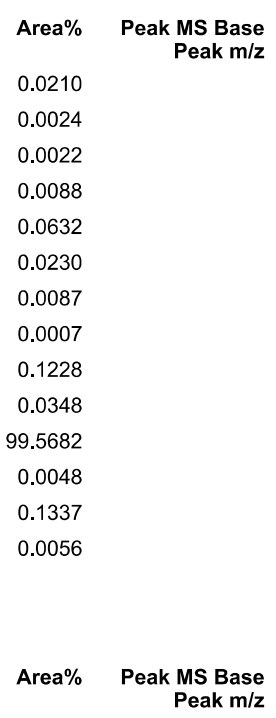

0.0210

0.0024

0.0022

0.0088

0.0632

0.0230

0.0087

0.0007

0.1228

0.0348

99.5682

0.0048

0.1337

0.0056

Area\% Peak MS Base

Peak $\mathrm{m} / \mathbf{z}$

100.0000

Signal: $\quad$ MS1 - TIC SCAN ESI Frag $=135 \mathrm{~V}$ Gain=1.0

RT [min] Type Width [min] Colo

Area

Height

$14.547 \mathrm{BB}$

1.2980

$\begin{array}{rr}60356072.3 & 4940172.45 \\ 140 & 14\end{array}$

Sum $\quad 60356072.3$
Area\% Peak MS Base

100.0000
Peak $\mathrm{m} / \mathbf{z}$ 

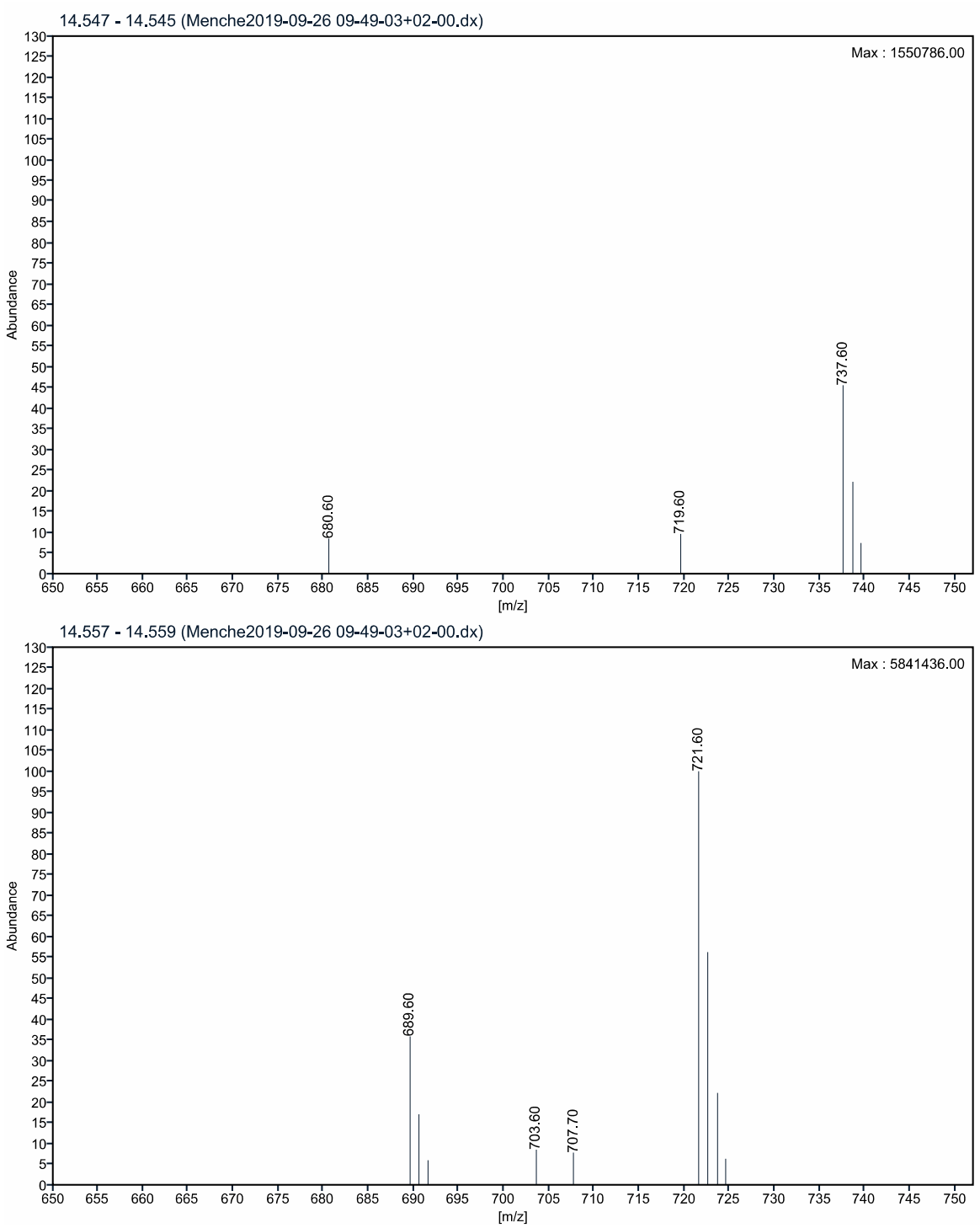

Figure S15. LC-MS spectrum of iso-archazolid $\mathrm{A}_{1}$. The purity of iso-archazolid $\mathrm{A}_{1}$ is $99.6 \%$ (retention time: 14.5 min corresponds to the desired compound) 
Sequence Name:

Data file:

Sample name:

Instrument:

Inj. volume:

Acq. method:

Processing method:
2019-09-26_Menche

Menche2019-09-26 11-21-54+02-00.dx Project Name:

iso-ArchazolidA2

Single Quad

5.000

0 wasser-acn standard 100-1000 AC-lon

MS_standard_plot.pmx
Single Quad

SYSTEM

2019-09-26 11:22:51+02:00

P1-A7
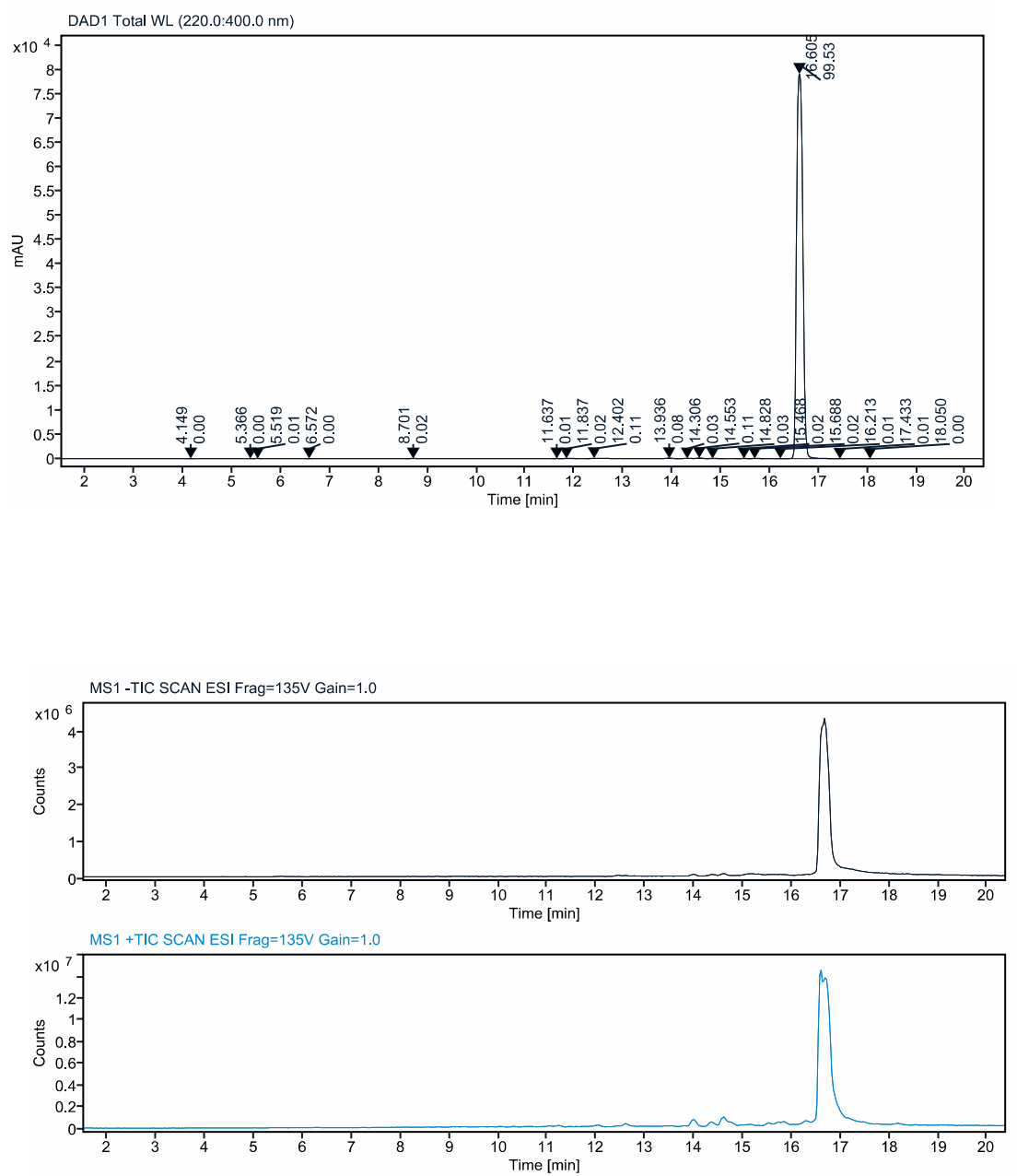
Signal: $\quad$ DAD1 Total WL $(220.0: 400.0 \mathrm{~nm})$

RT [min] Type Width [min] Colo

4.149 MM m

$5.366 \mathrm{MM} \mathrm{m}$

$5.519 \mathrm{MM} \mathrm{m}$

$6.572 \mathrm{MM} \mathrm{m}$

$8.701 \mathrm{MM} \mathrm{m}$

$11.637 \mathrm{MM} \mathrm{m}$

$11.837 \mathrm{MM} \mathrm{m}$

$12.402 \mathrm{MM} \mathrm{m}$

$13.936 \mathrm{MM} \mathrm{m}$

$14.306 \mathrm{MM} \mathrm{m}$

$14.553 \mathrm{MM} \mathrm{m}$

$14.828 \mathrm{MM} \mathrm{m}$

$15.468 \mathrm{MM} \mathrm{m}$

$16.213 \mathrm{MM} \mathrm{m}$

16.605 BB

$17.433 \mathrm{MM} \mathrm{m}$

$18.050 \mathrm{MM} \mathrm{m}$

\subsection{7}

0.0000

0.0740

0.0942

0.0967

0.0784

0.1249

0.1769

0.1114

0.0991

0.1144

0.1366

0.1048

0.1864

0.1183

1.0200

0.0835

0.0864
$15.688 \mathrm{MM} m$

Sum 703899.7995

Signal: $\quad$ MS1 +TIC SCAN ESI Frag=135V Gain=1.0

RT [min] Type Width [min] Colo

$$
\begin{aligned}
& \text { 16.597 MM m } \\
& 0.2085 \\
& \begin{array}{rr}
217989242 . \\
8519
\end{array} \\
& \text { Sum } 217989242 .
\end{aligned}
$$

Area

Signal: $\quad$ MS1 - TIC SCAN ESI Frag $=135 \mathrm{~V}$ Gain $=1.0$

RT [min] Type Width [min] Colo

Area

$16.669 \mathrm{BB}$

2.0436

$\begin{array}{rr}63642714.4 & 4246393.58 \\ 491 & 67\end{array}$

Sum 63642714.4
Height

Area\%

100.0000

$\begin{array}{rr}\text { Height } & \text { Area\% } \\ 2.7019 & 0.0027 \\ 0.0000 & 0.0000 \\ 14.4850 & 0.0099 \\ 3.9381 & 0.0030 \\ 24.3769 & 0.0198 \\ 8.2608 & 0.0055 \\ 17.5549 & 0.0216 \\ 58.3465 & 0.1058 \\ 85.0639 & 0.0823 \\ 37.5297 & 0.0307 \\ 109.3898 & 0.1071 \\ 24.2982 & 0.0304 \\ 16.9291 & 0.0150 \\ 11.7429 & 0.0187 \\ 10.7728 & 0.0108 \\ 79128.3324 & 99.5279 \\ 7.6944 & 0.0056 \\ 4.8457 & 0.0033\end{array}$

eak MS Base

Peak m/z

.0000

0.0099

0.0055

.0216

0.0823

.0307

0.0150

0.0187

9.5279

0.0033

Peak MS Base

Peak m/z 



Figure S16. LC-MS spectrum of iso-archazolid $\mathrm{A}_{2}$. The purity of iso-archazolid $\mathrm{A}_{2}$ is $99.5 \%$ (retention time: 16.6 min corresponds to the desired compound) 
Sequence Name:

Data file:

Sample name:

Instrument:

Inj. volume:

Acq. method:

Processing method:
2019-09-26_Menche

Menche2019-09-26 11-52-53+02-00.dx Project Name:

Desmerthyl-ArchazolidF

Single Quad

5.000

0 wasser-acn standard 100-1000 AC-

MS_standard_plot.pmx
Single Quad

SYSTEM

2019-09-26 11:53:47+02:00

P1-A8
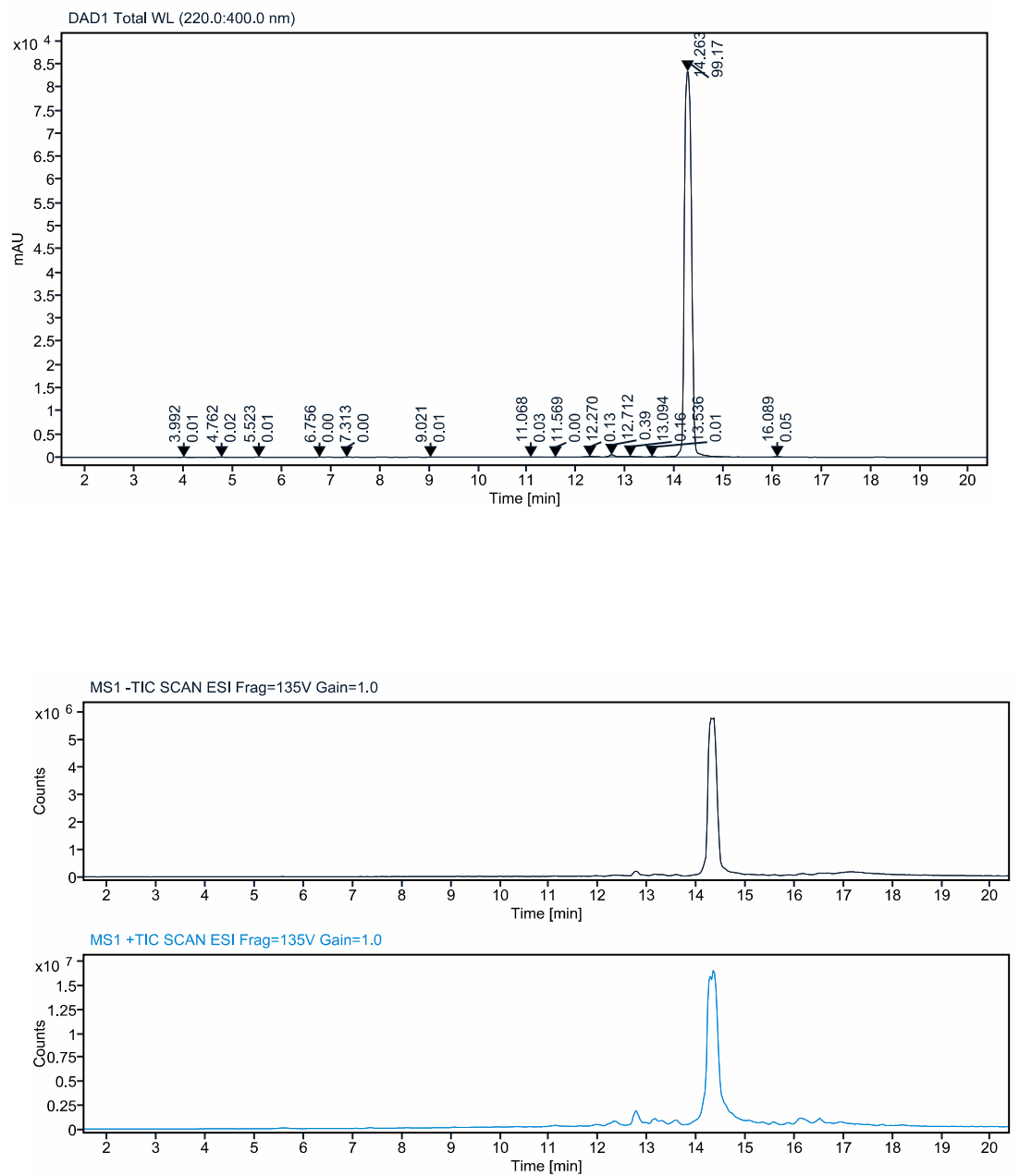
Signal: $\quad$ DAD1 Total WL $(220.0: 400.0 \mathrm{~nm})$

\section{RT [min] Type Width [min] Colo}

$3.992 \mathrm{MM} \mathrm{m}$

$4.762 \mathrm{MM} \mathrm{m}$

$5.523 \mathrm{MM} \mathrm{m}$

$6.756 \mathrm{MM} \mathrm{m}$

$7.313 \mathrm{MM} \mathrm{m}$

$9.021 \mathrm{MM} \mathrm{m}$

$11.068 \mathrm{MM} \mathrm{m}$

$11.569 \mathrm{MM} \mathrm{m}$

$12.270 \mathrm{MM} \mathrm{m}$

$12.712 \mathrm{MM} \mathrm{m}$

$13.094 \mathrm{MM} \mathrm{m}$

$13.536 \mathrm{MM} \mathrm{m}$

14.263 BB

16.089 MM m

0.1744
0.1139
0.0721
0.0798
0.0835
0.1013
0.1626
0.0818
0.1670
0.1124
0.1457
0.0682
1.2413
0.1456

Are

181.6215

47.4648

24.1112

29.6648

89.4195

218.2497

28.4352

1091.5083

3241.3196

1325.4339

124.7040 $828138.6658 \quad 83420.9207$

$458.0777 \quad 55.8873$

Sum 835090.9425

Height

7.4553

24.6675

10.2395

5.0425

6.0233

14.2291

17.8501

6.1681

90.3143

470.2219

131.4142

40.1551
Signal: $\quad$ MS1 +TIC SCAN ESI Frag=135V Gain=1.0

RT [min] Type Width [min] Colo

Area

14.346 MM m

0.2424

229759544 6122

Sum 229759544

Height

15217685.3

941

Signal: $\quad$ MS1 - TIC SCAN ESI Frag $=135 \mathrm{~V}$ Gain=1.0

RT [min] Type Width [min] Colo

Area

Height

14.339 MM m

0.2013

$\begin{array}{rr}69350417.0 & 5647174.19 \\ 358 & 33\end{array}$

Sum $\begin{array}{r}69350417.0 \\ 358\end{array}$

$$
\begin{array}{r}
\text { Area\% } \\
0.0110 \\
0.0217 \\
0.0057 \\
0.0029 \\
0.0036 \\
0.0107 \\
0.0261 \\
0.0034 \\
0.1307 \\
0.3881 \\
0.1587 \\
0.0149 \\
99.1675 \\
0.0549
\end{array}
$$

Area\% Peak MS Base

Peak m/z

100.0000

Area\% Peak MS Base

100.0000
Peak MS Base
Peak m/z 

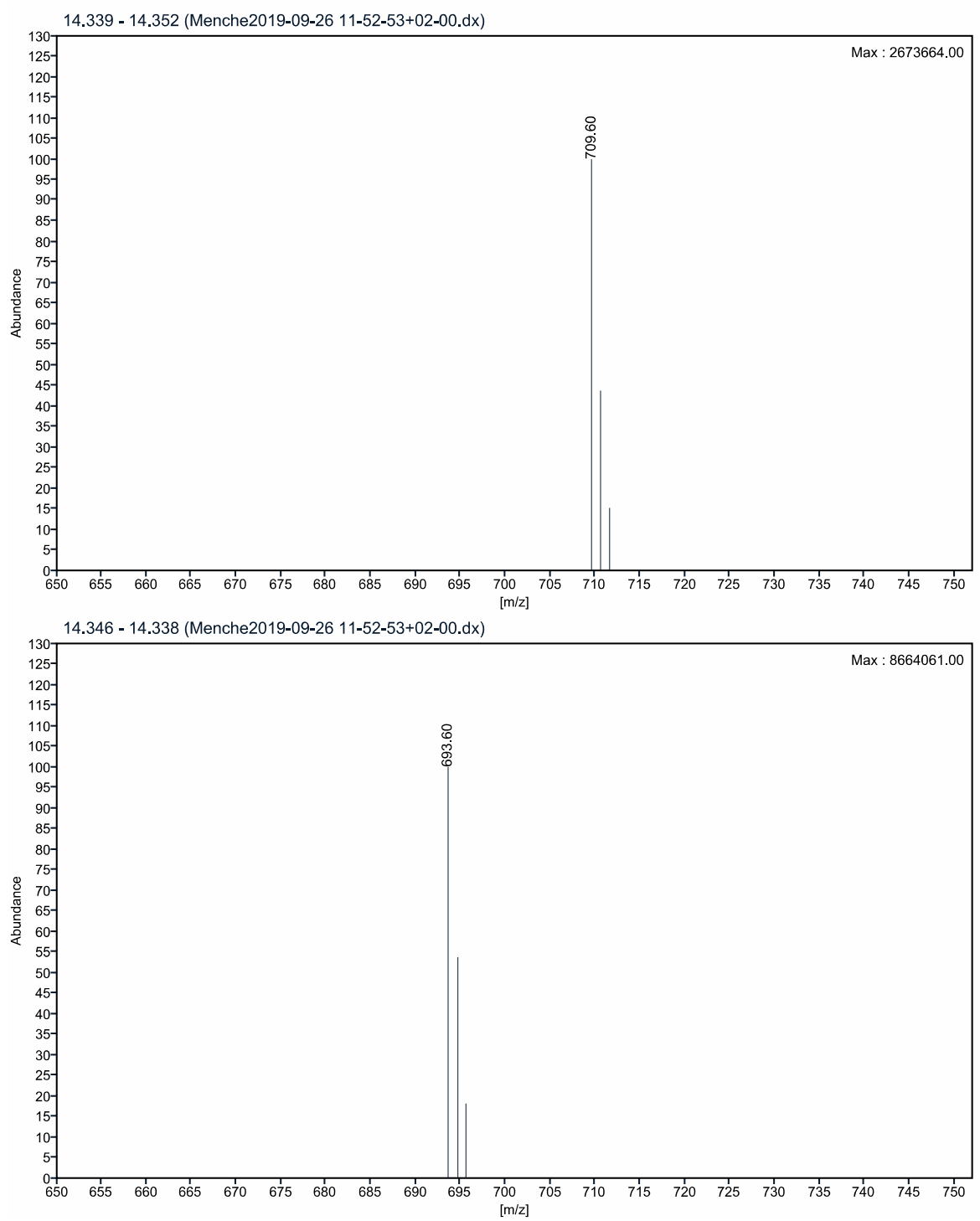

Figure S17. LC-MS spectrum of desmethyl archazolid F. The purity of desmethyl archazolid F is $99.2 \%$ (retention time: 14.3 min corresponds to the desired compound) 
Sequence Name:

Data file:

Sample name:

Instrument:

Inj. volume:

Acq. method:

Processing method:
2019-09-26_Menche

Menche2019-09-26 10-50-58+02-00.dx Project Name:

ArchazolidF Methylester

Single Quad

5.000

0 wasser-acn standard 100-1000 AC-

MS_standard_plot.pmx
Single Quad

SYSTEM

2019-09-26 10:51:52+02:00

P1-A6

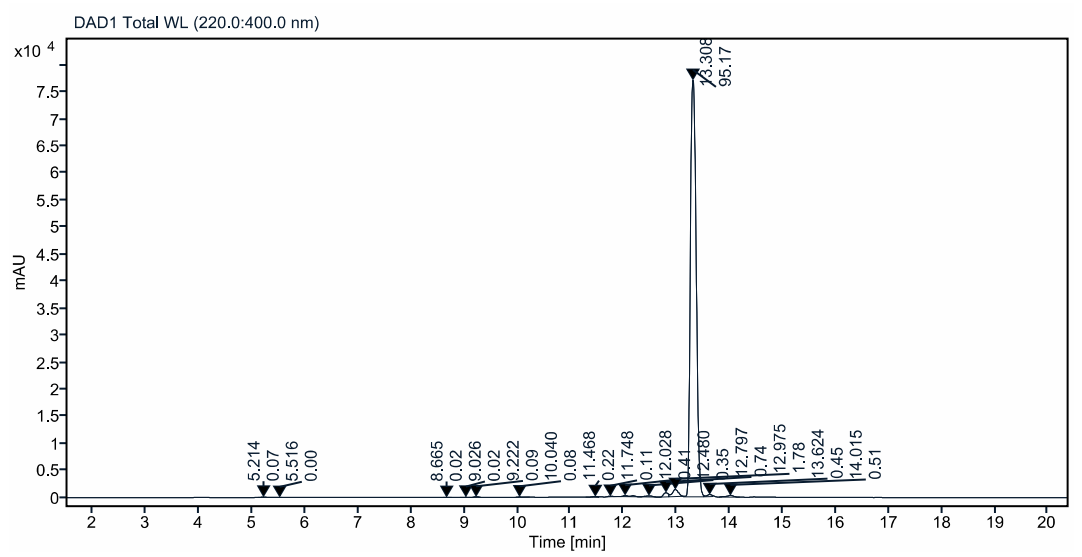

MS1 - TIC SCAN ESI Frag=135V Gain $=1.0$
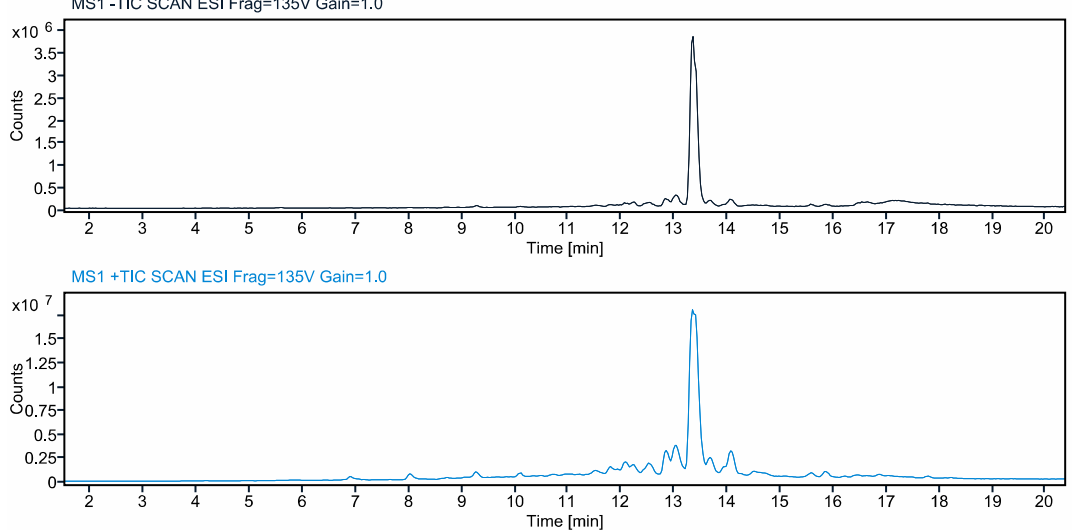
Signal: $\quad$ DAD1 Total WL $(220.0: 400.0 \mathrm{~nm})$

RT [min] Type Width [min] Colo

$\begin{array}{rl}5.214 \mathrm{MM} \mathrm{m} & 0.1059 \\ 5.516 \mathrm{MM} \mathrm{m} & 0.0466 \\ 8.665 \mathrm{MM} \mathrm{m} & 0.0793 \\ 9.026 \mathrm{MM} \mathrm{m} & 0.0854 \\ 9.222 \mathrm{MM} \mathrm{m} & 0.0869 \\ 10.040 \mathrm{MM} \mathrm{m} & 0.1047 \\ 11.468 \mathrm{MM} \mathrm{m} & 0.1226 \\ 11.748 \mathrm{MM} \mathrm{m} & 0.1028 \\ 12.028 \mathrm{MM} \mathrm{m} & 0.1665 \\ 12.480 \mathrm{MM} \mathrm{m} & 0.1460 \\ 12.797 \mathrm{MM} \mathrm{m} & 0.0929 \\ 12.975 \mathrm{MM} \mathrm{m} & 0.1262 \\ 13.308 \mathrm{MM} \mathrm{m} & 0.1221 \\ 13.624 \mathrm{MM} \mathrm{m} & 0.1126 \\ 14.015 \mathrm{MM} \mathrm{m} & 0.1440\end{array}$

\section{Area}

426.9487

26.4974

99.9880

116.8954

536.6989

463.1888

1322.3862

669.2934

2508.1132

2126.1312

4494.3502

10894.3234

581757.4324

2740.9095

3091.1655

Sum 611274.3223

Signal: $\quad$ MS1 + TIC SCAN ESI Frag=135V Gain=1.0

RT [min] Type Width [min] Colo

Area

$13.350 \mathrm{MM} \mathrm{m}$

0.1480

$\begin{array}{rrr} & 173071221 . & 16396363.5 \\ & 6774 & 743 \\ \text { Sum } & 173071221 . & \end{array}$

Signal: $\quad$ MS1 - TIC SCAN ESI Frag $=135 \mathrm{~V}$ Gain $=1.0$

\begin{abstract}
RT [min] Type Width [min] Colo
\end{abstract}
13.349 BV

\begin{abstract}
0.4019
\end{abstract}

Height

65.6047
10.8175
20.3533
22.9567
102.7790
66.9269
163.0819
99.1235
208.2812
217.6669
783.3375
1320.8735
7011.5028
405.5269
321.8545

321.8545

$$
6774
$$

Area

3767934.78
30

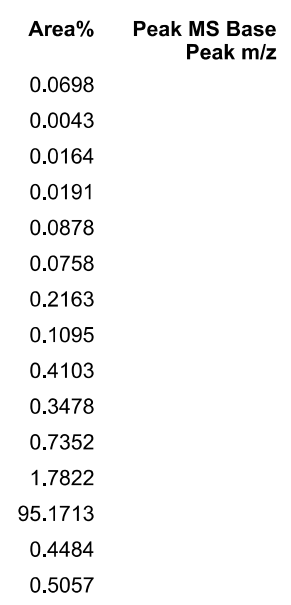

Area\%

100.0000

Peak MS Base Peak m/z

Area\%

100.0000 

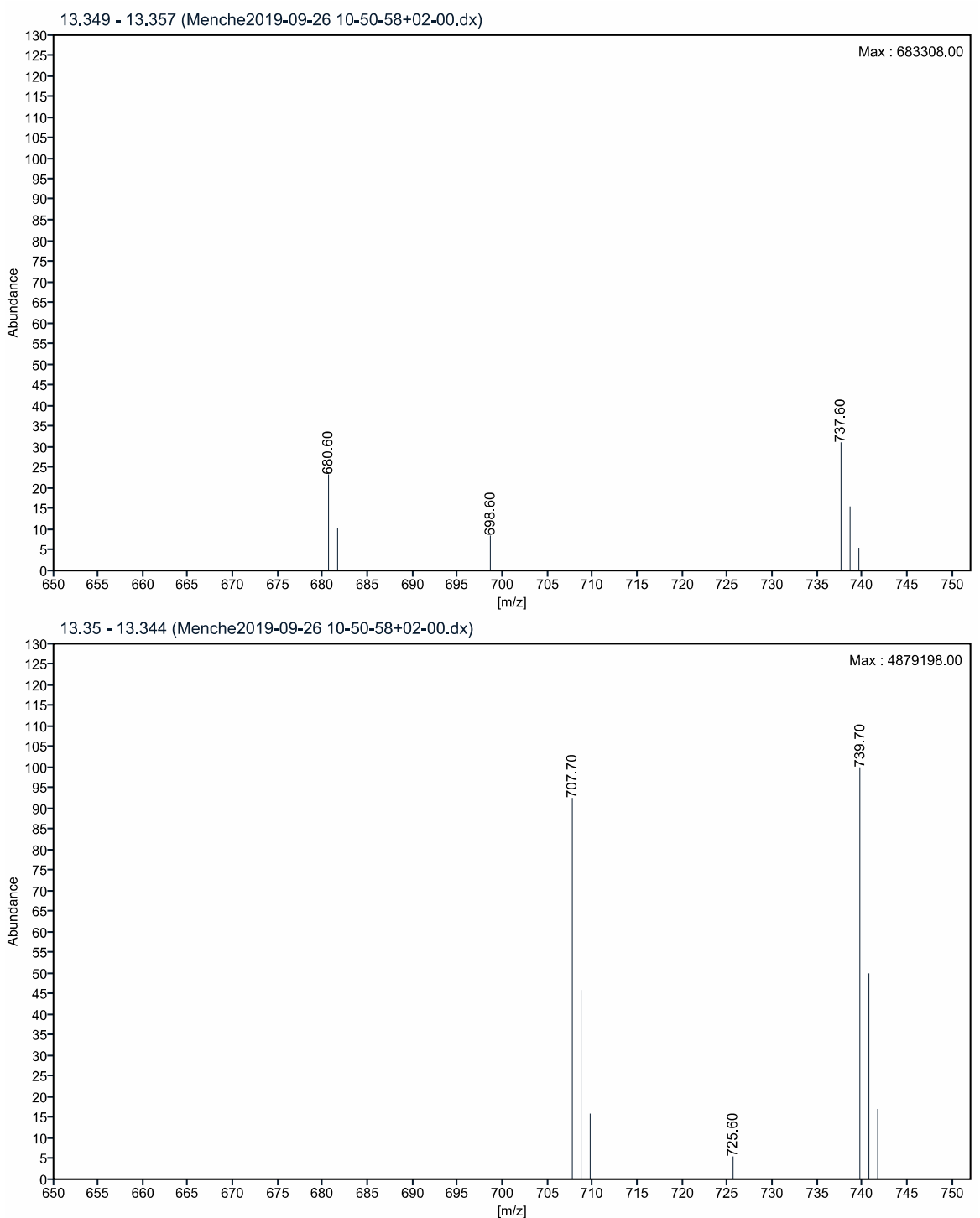

Figure S18. LC-MS spectrum of archazolid F methylester. The purity of archazolid F methylester is $95.2 \%$ (retention time: 13.3 min corresponds to the desired compound) 


\section{Copies of NMR spectra}

- NMR Spectra start on the next page - 
NMR-Spectra for Compound 3

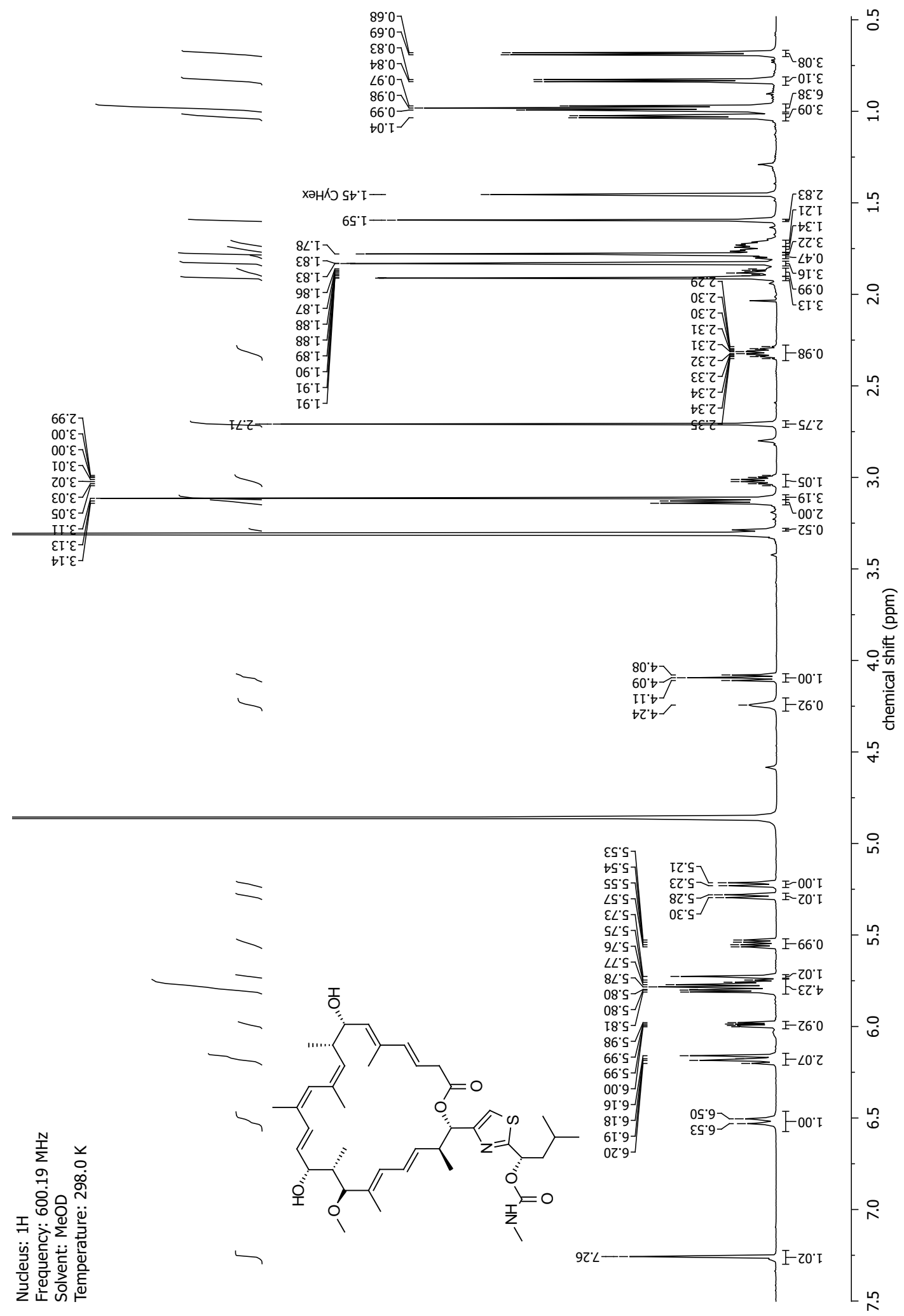




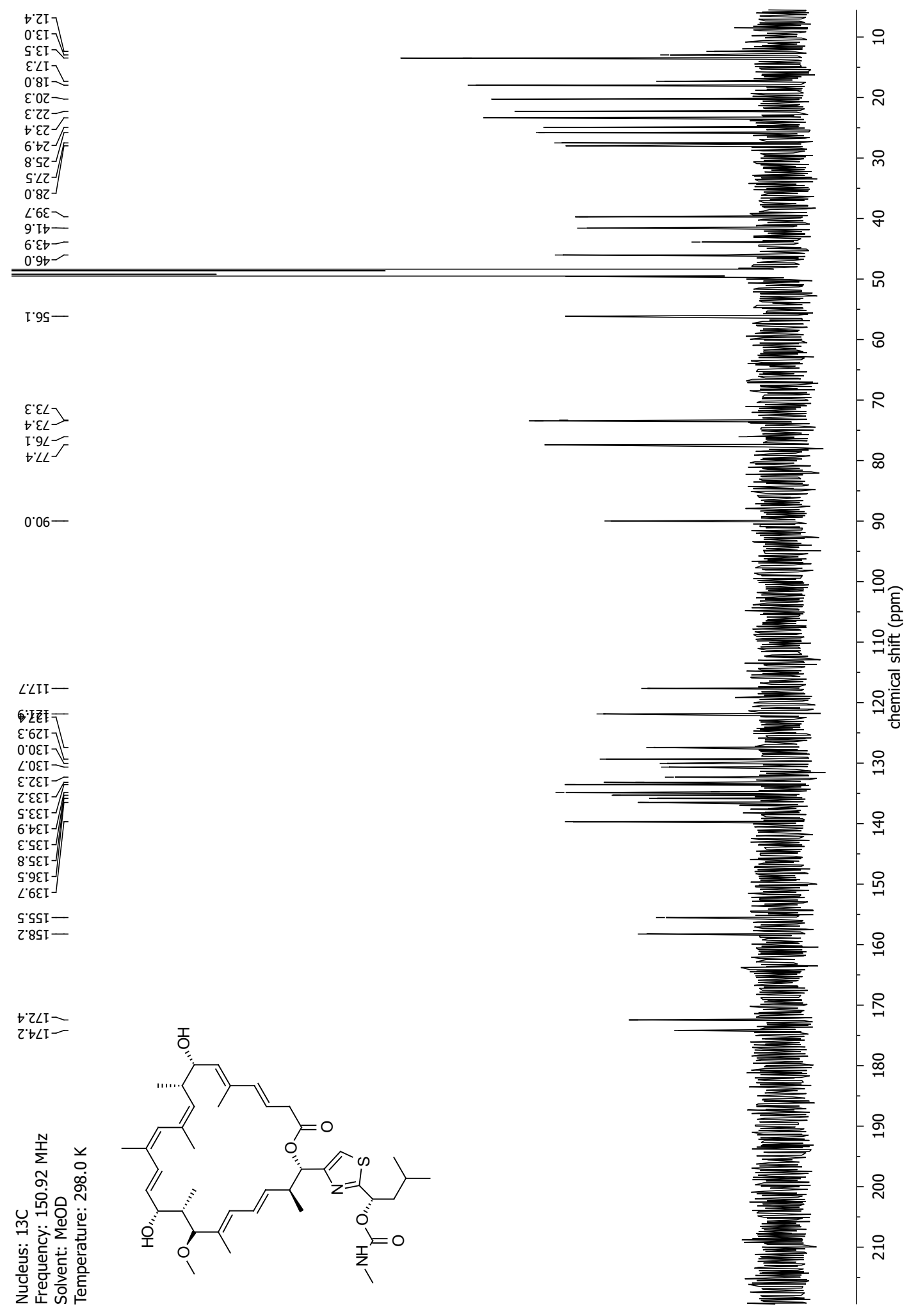




\section{NMR-Spectra for Compound 4}

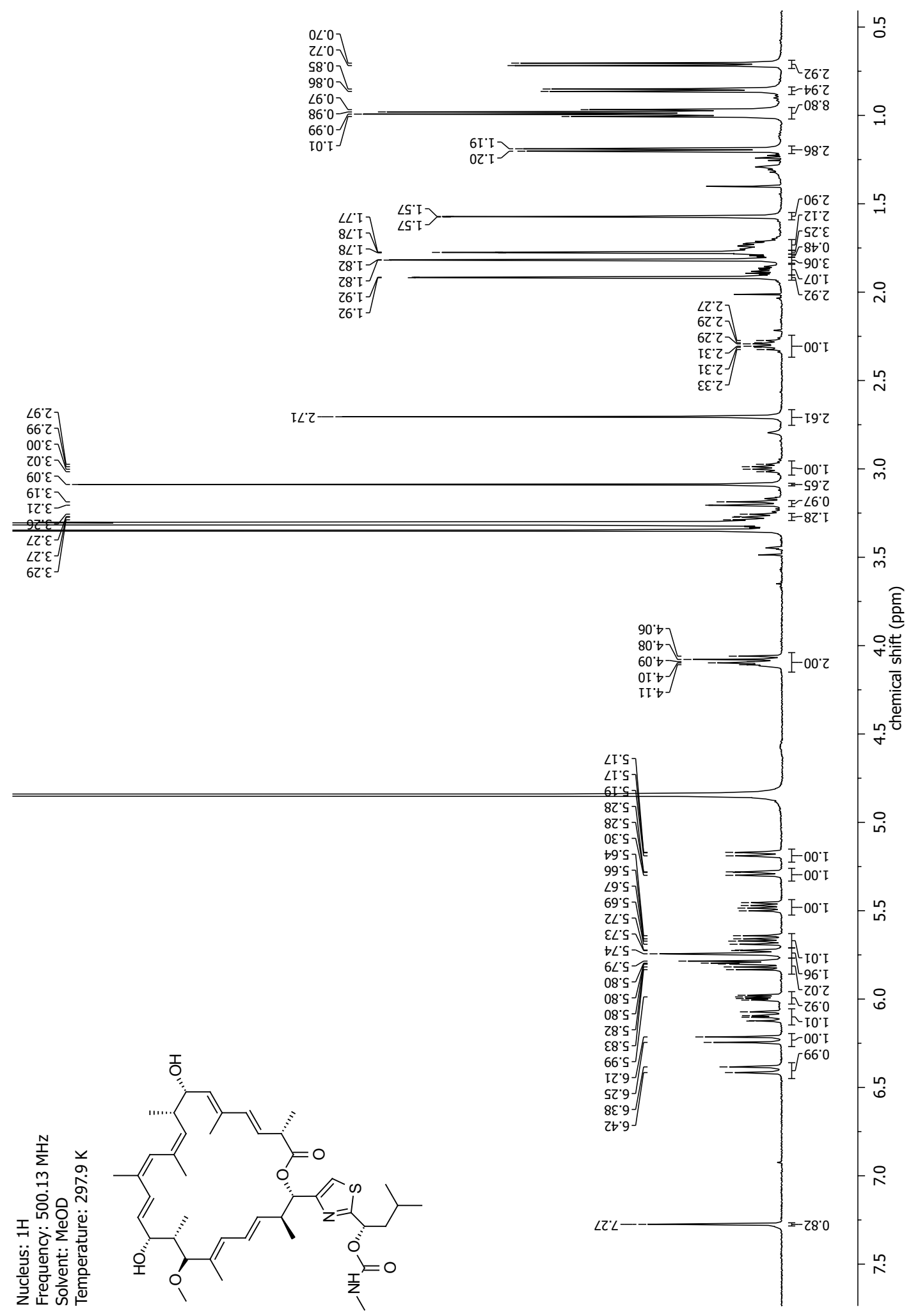



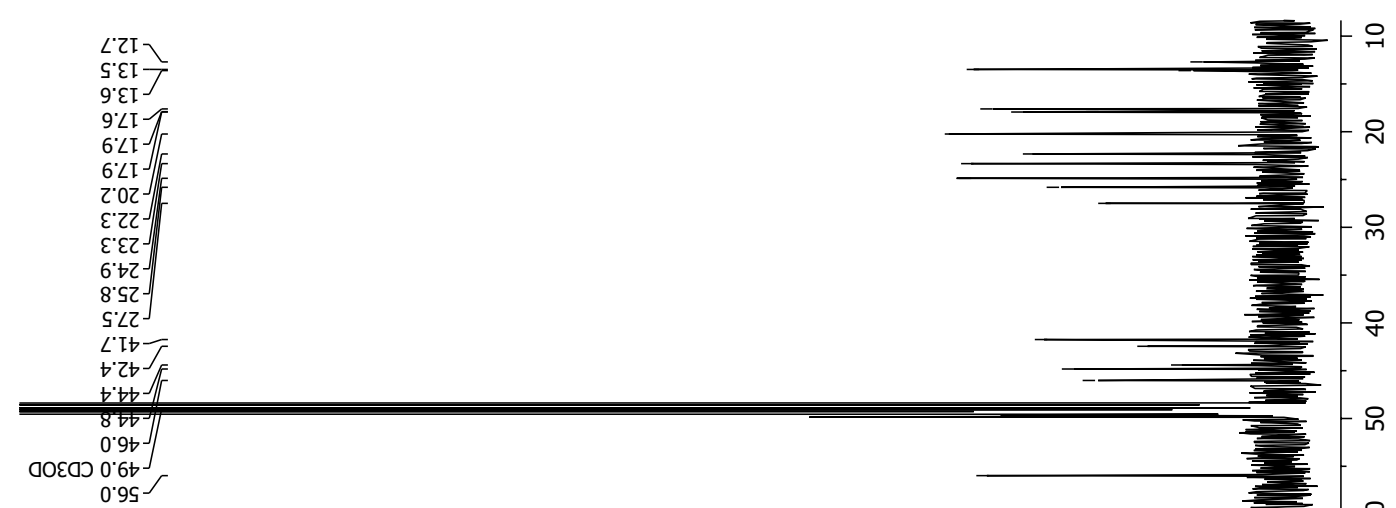

$\varepsilon \cdot \varepsilon L \backslash$
$\dagger \cdot \varepsilon L \backslash$
$t^{\prime} L L-$

0.06-

S'LII -

L'LZI

I'6ZI]

S.6ZI -

6.6ZI /F

I'IEI

乙'हعI

scter

sw

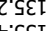

6'şr

Eंตहा

LLEI

L'SSI I ISI

6'\&LI-

$9^{\prime} \varsigma L \mathrm{~L}=$
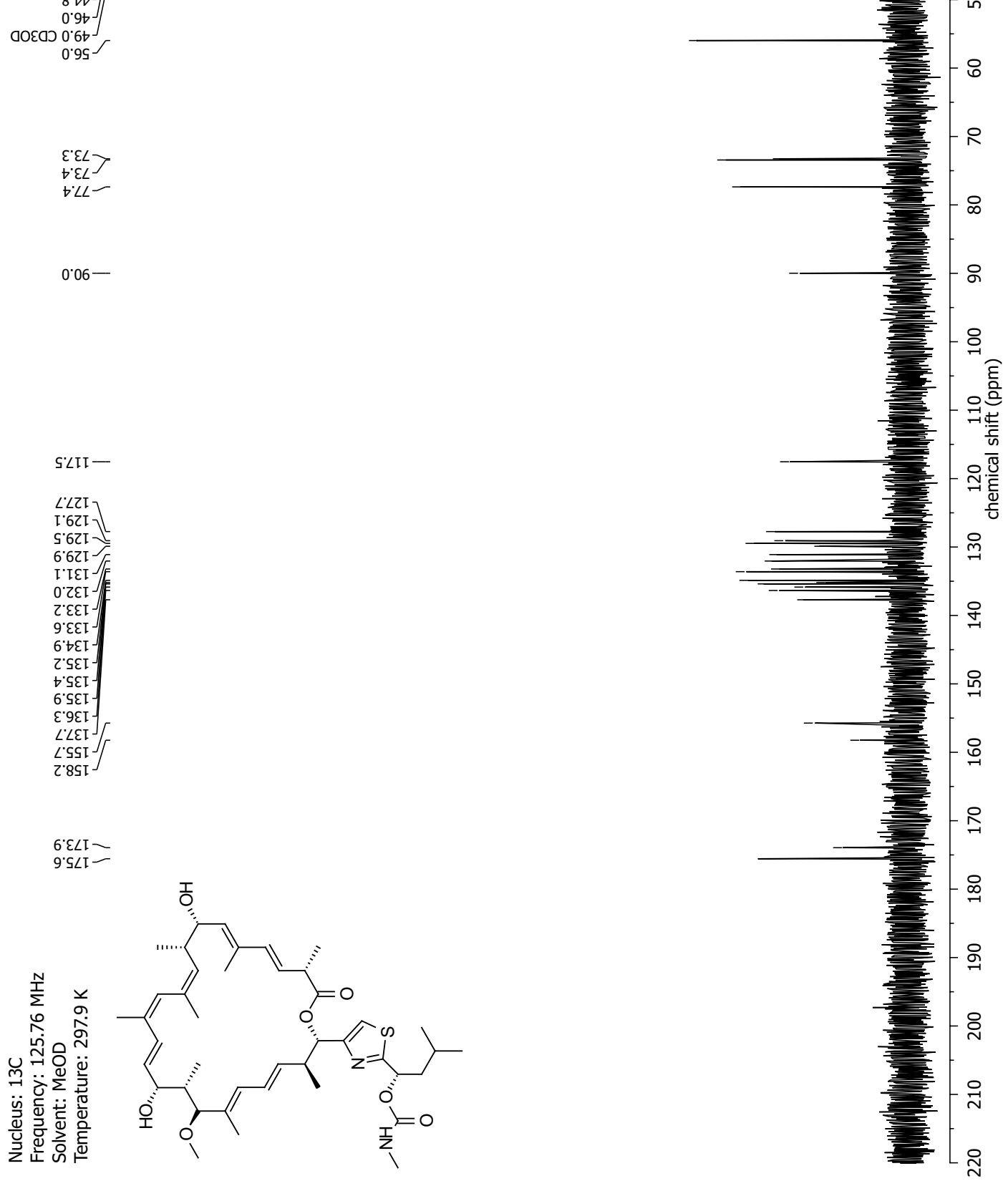


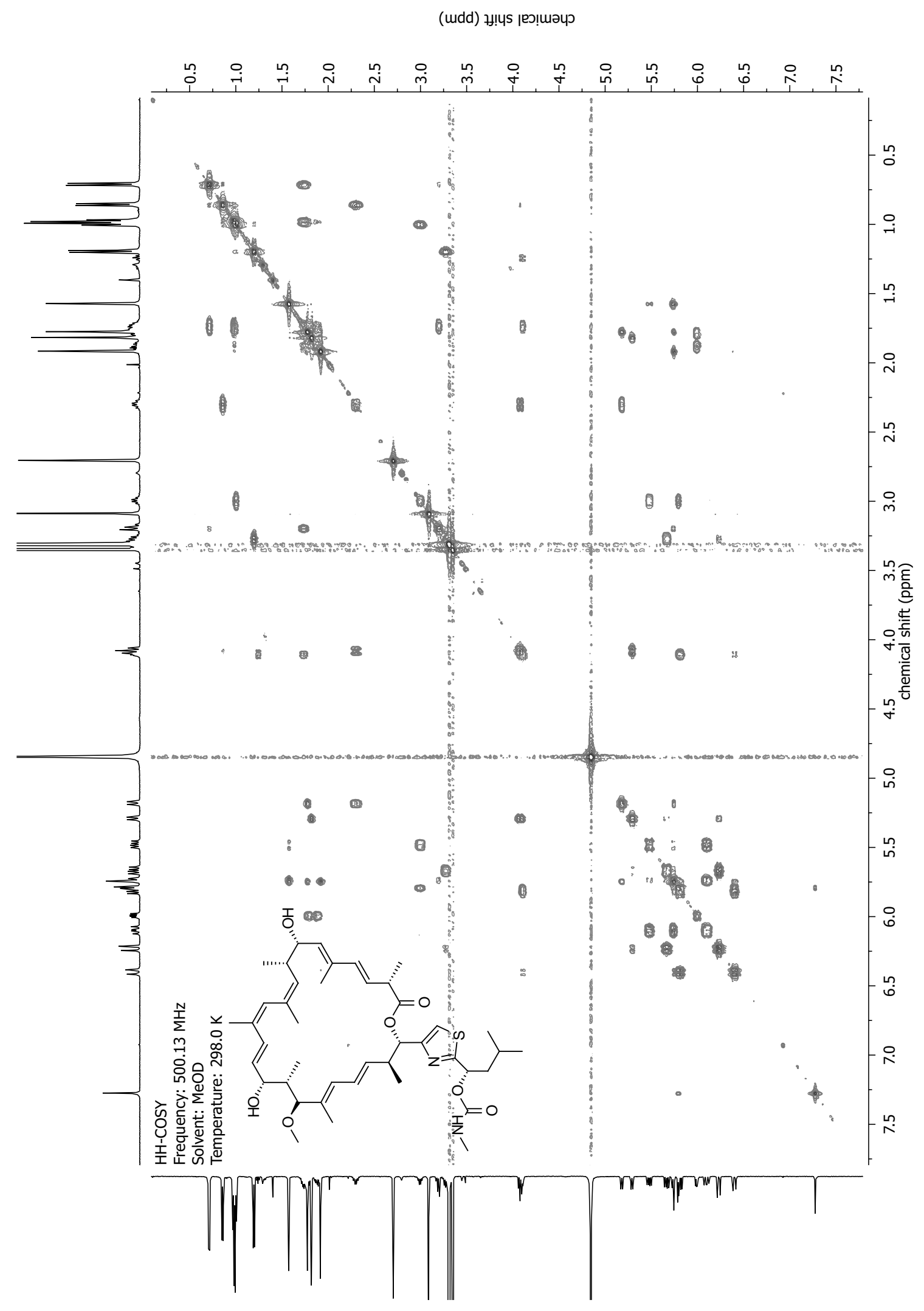




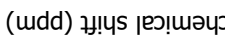

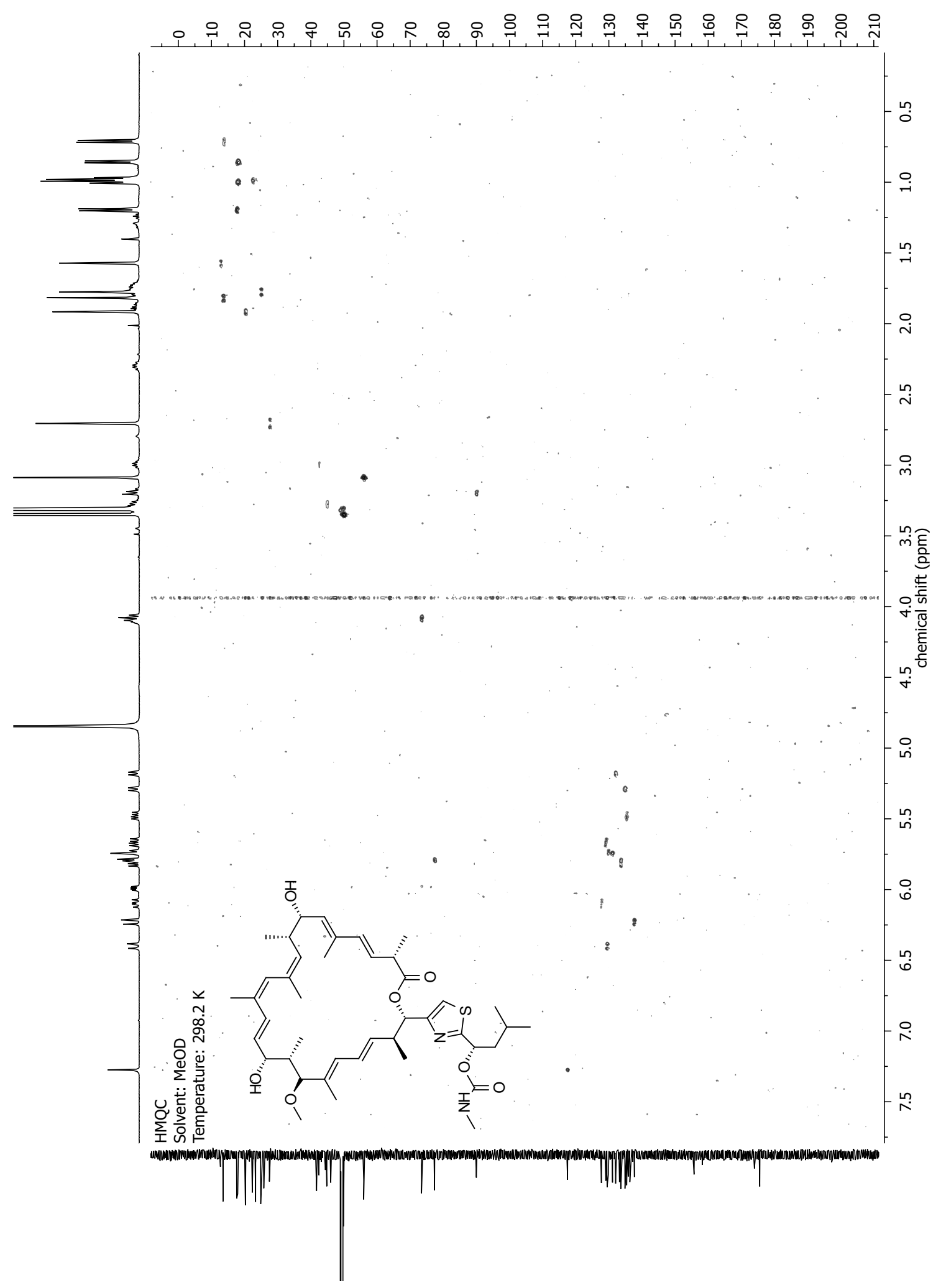


(udd) भ!บऽ ןอว!นวцว

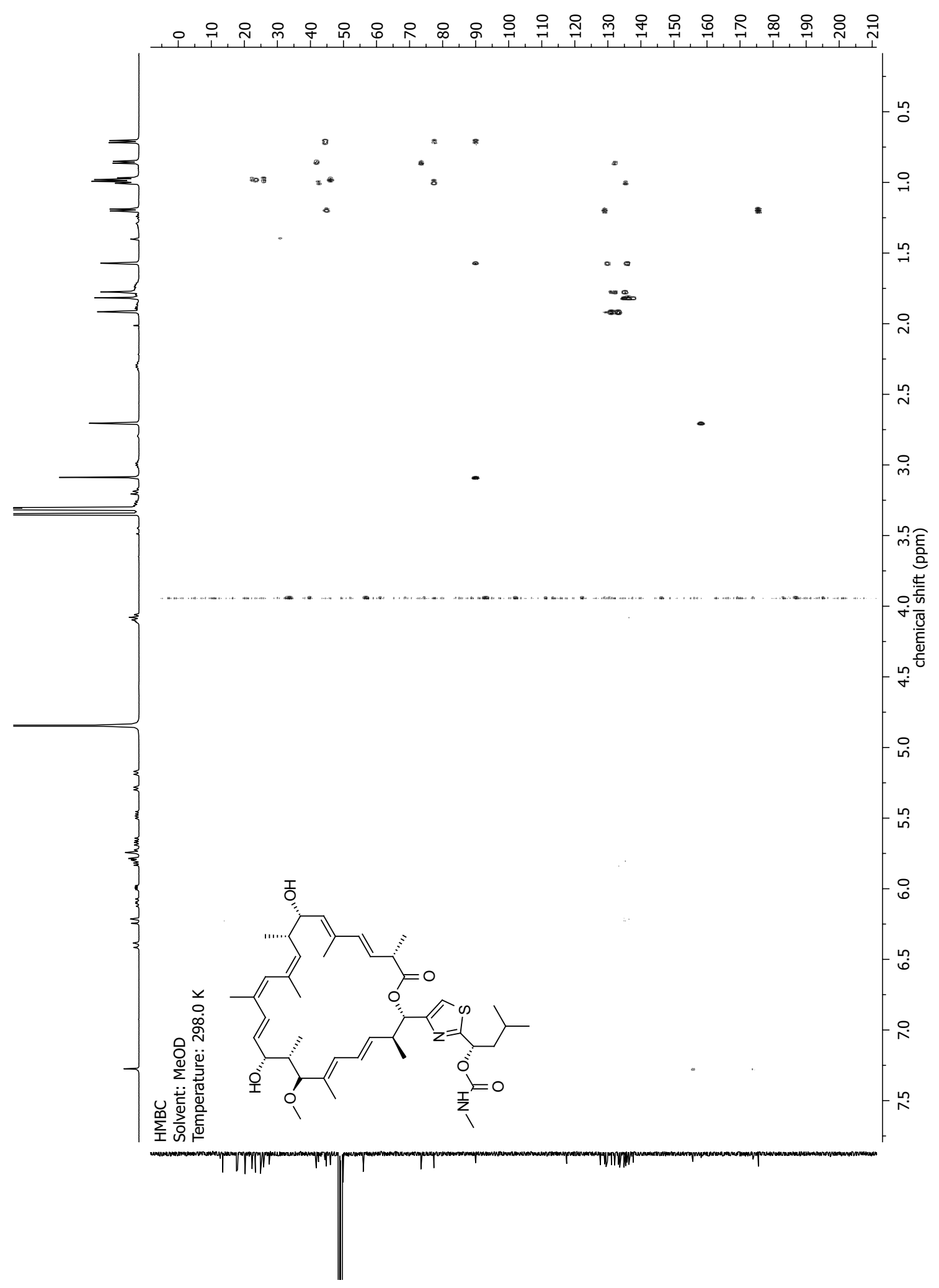




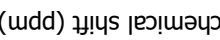

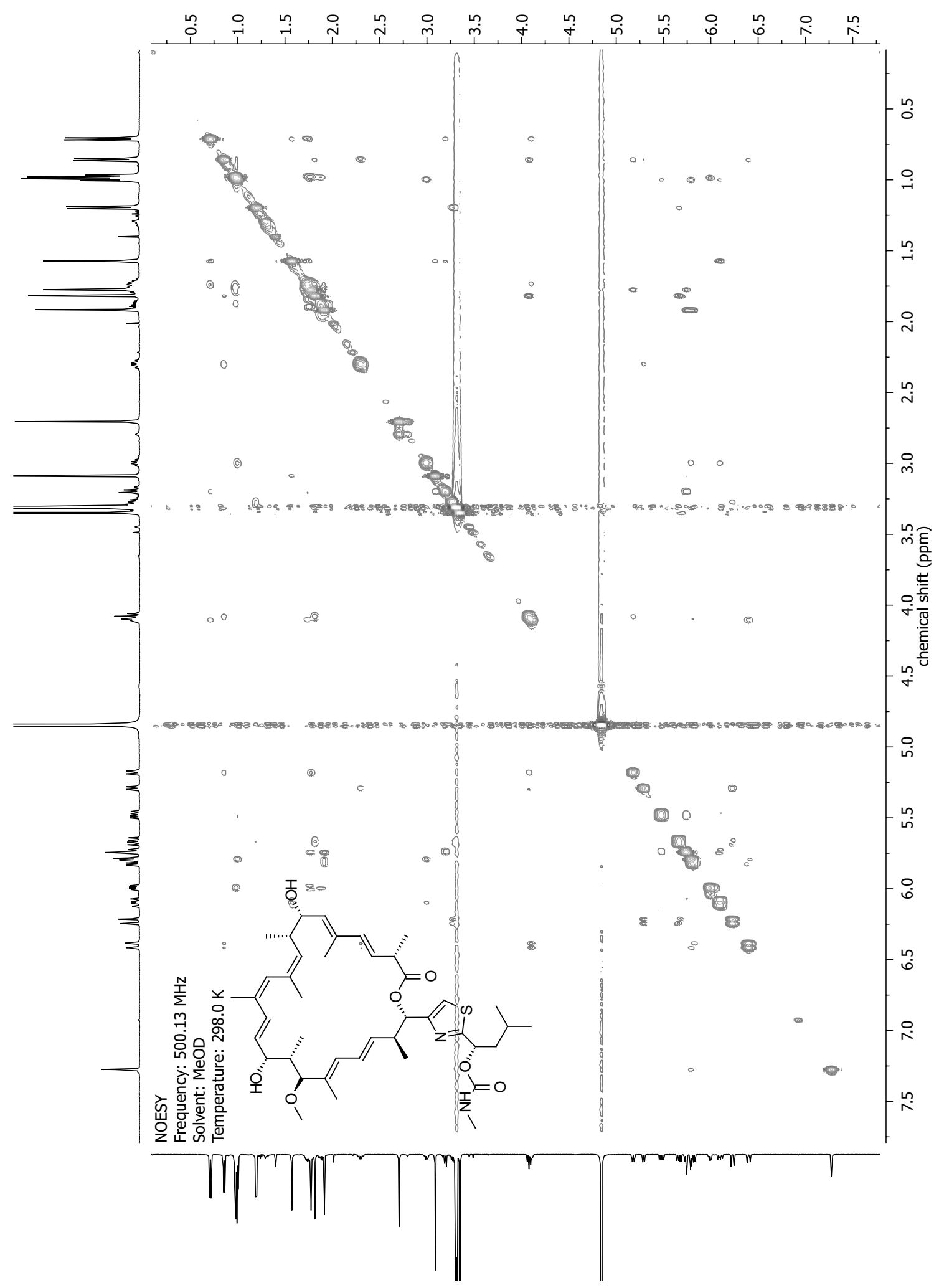




\section{NMR-Spectra for Compound 5}

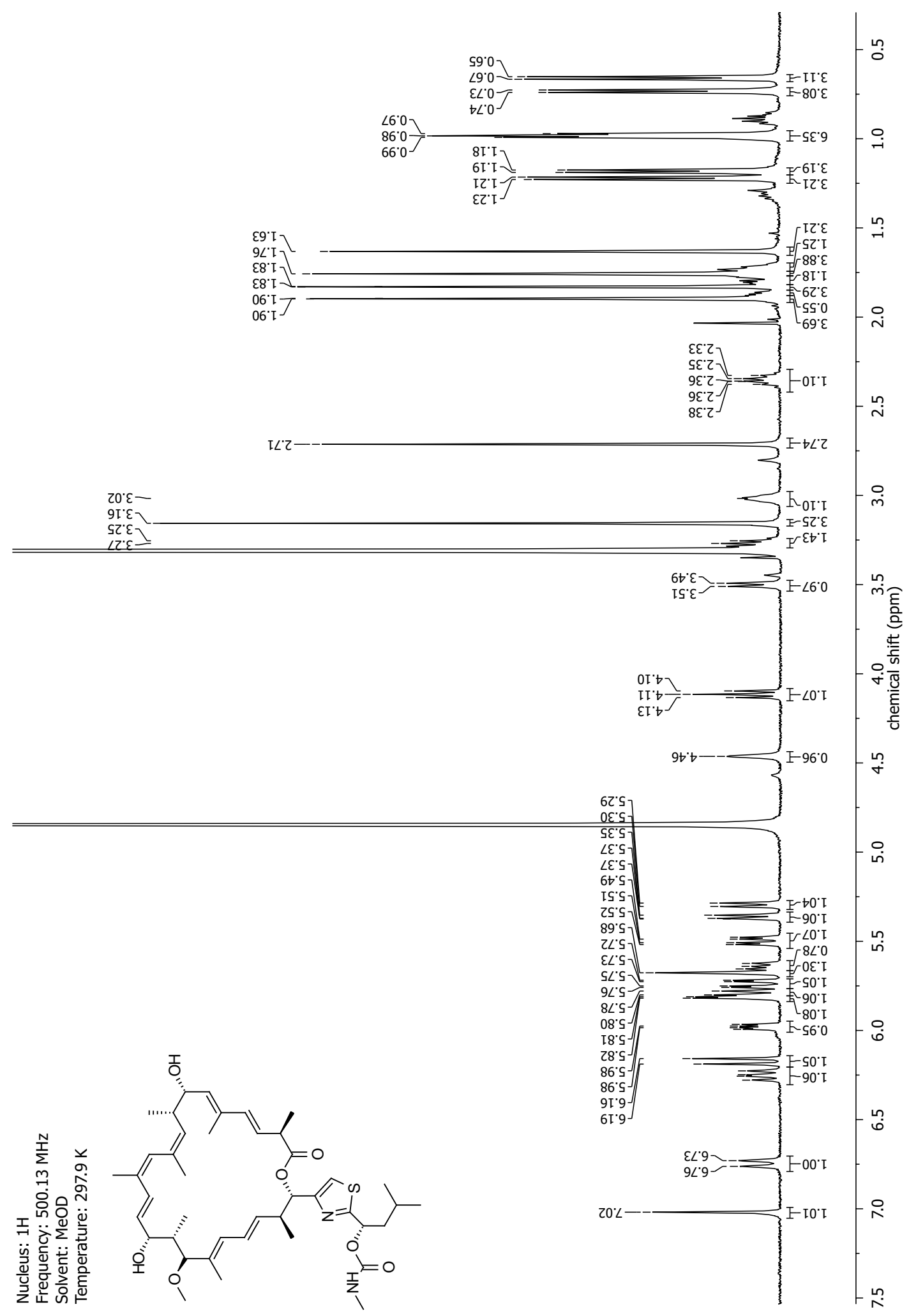



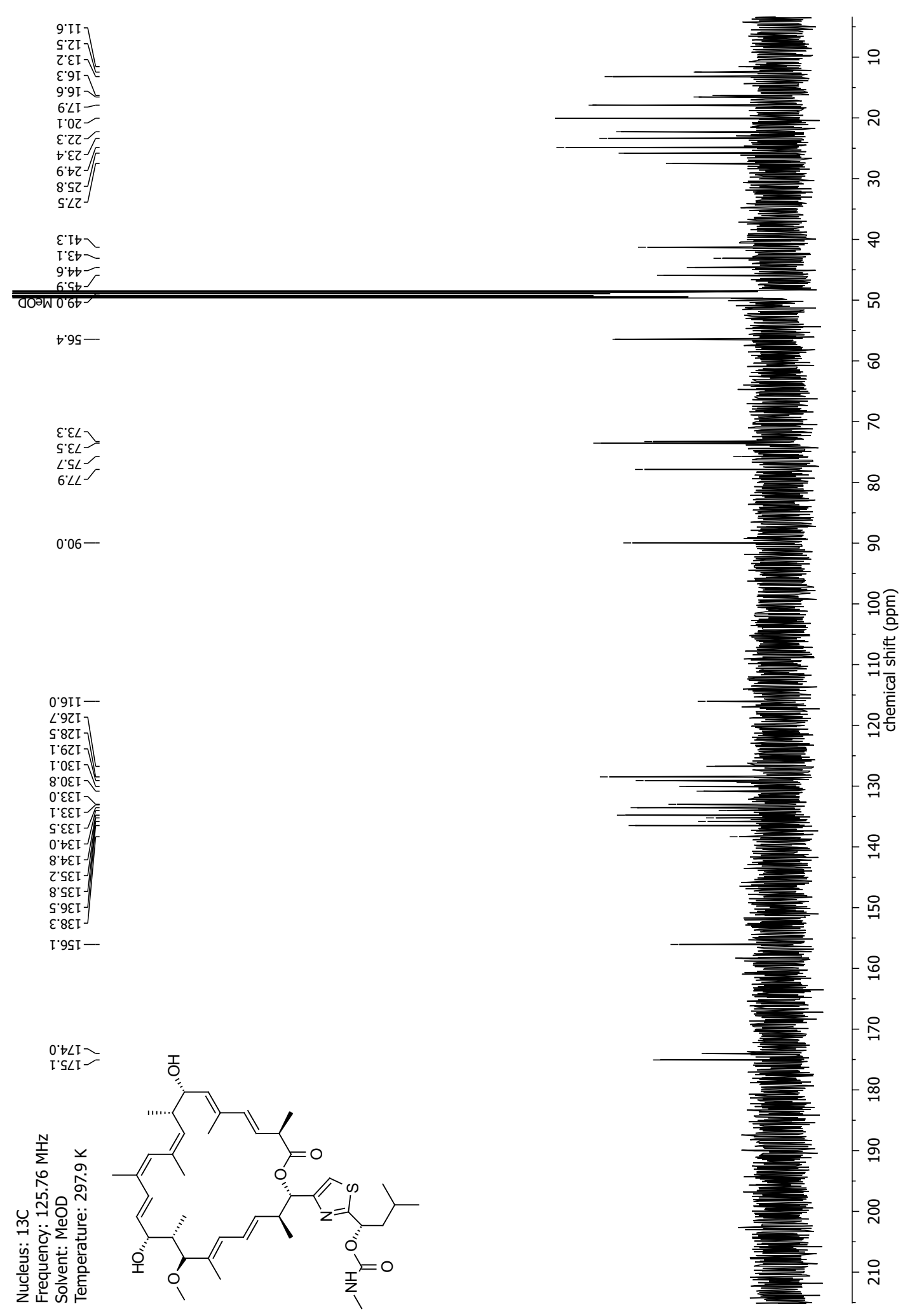


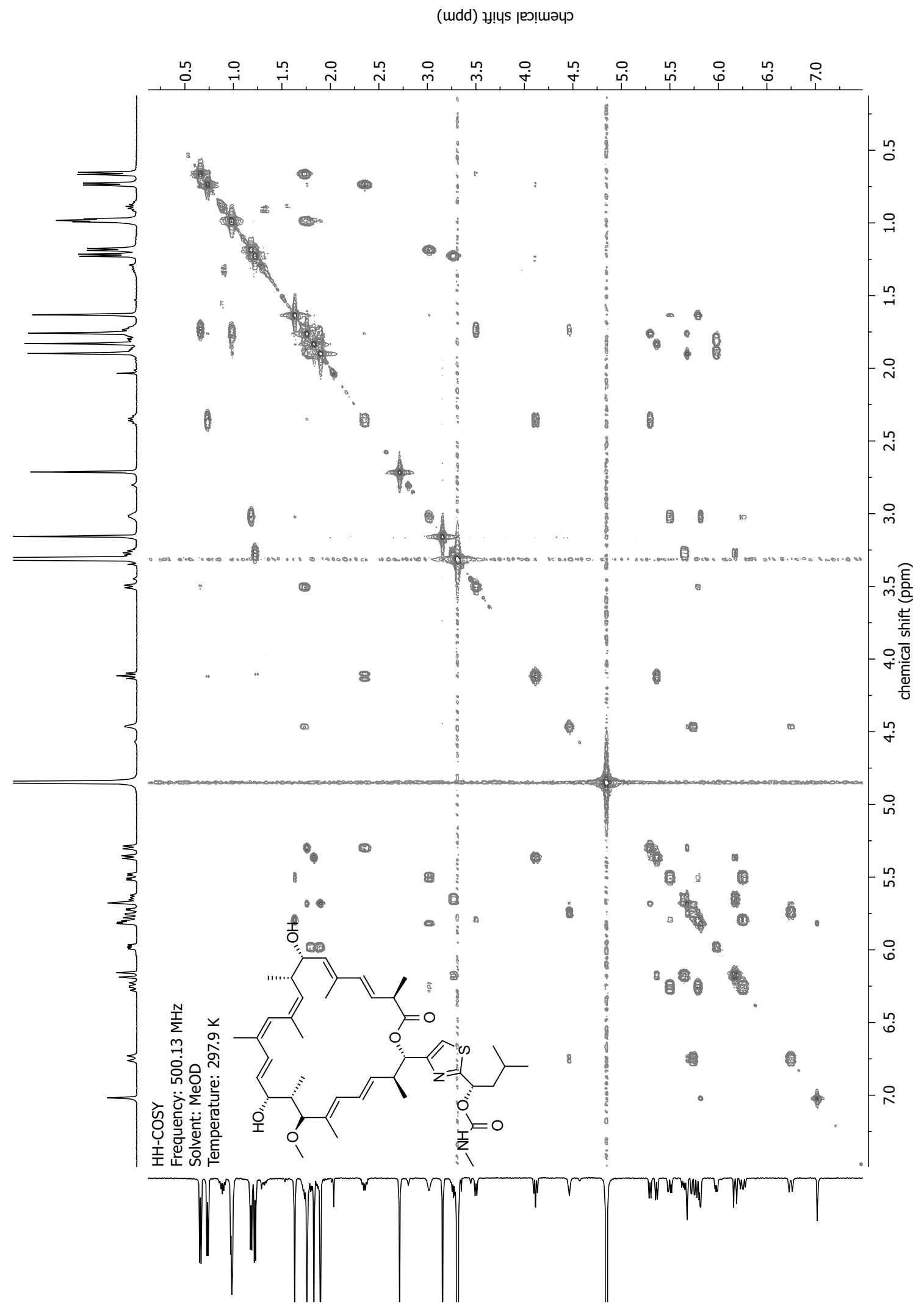




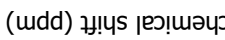

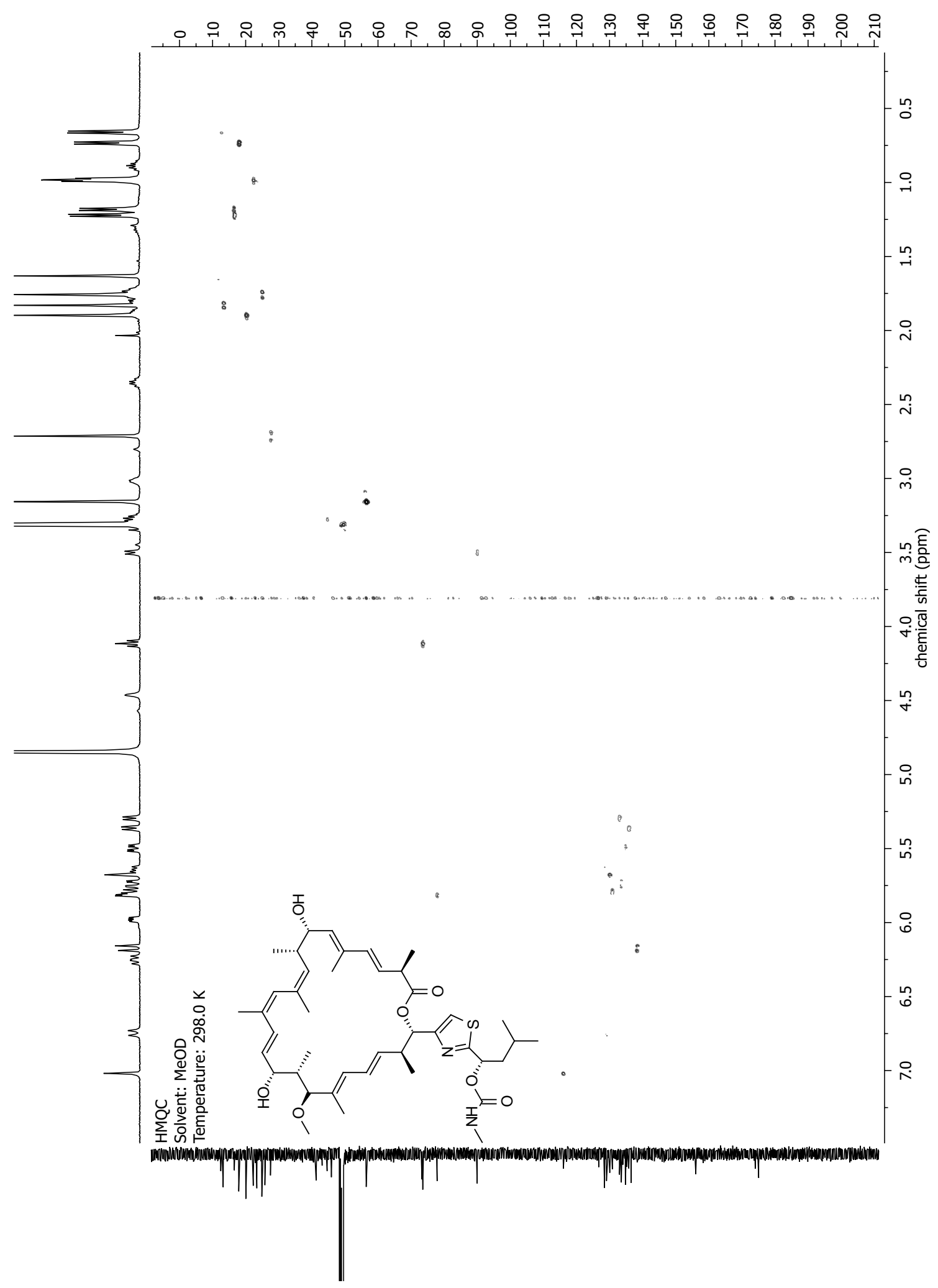


(udd) भ!!पs ןеग!шәчว

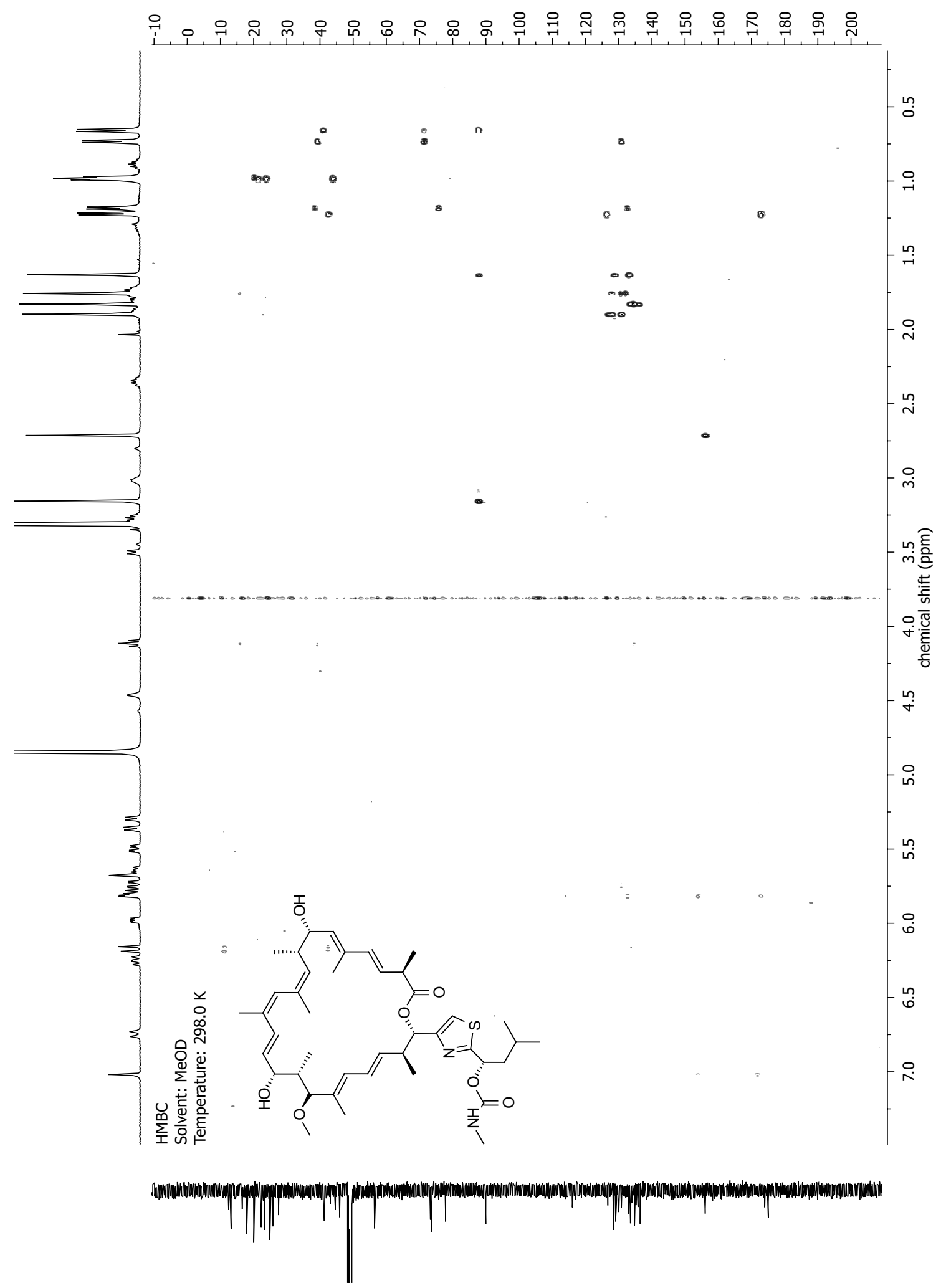







NMR-Spectra for Compound 9

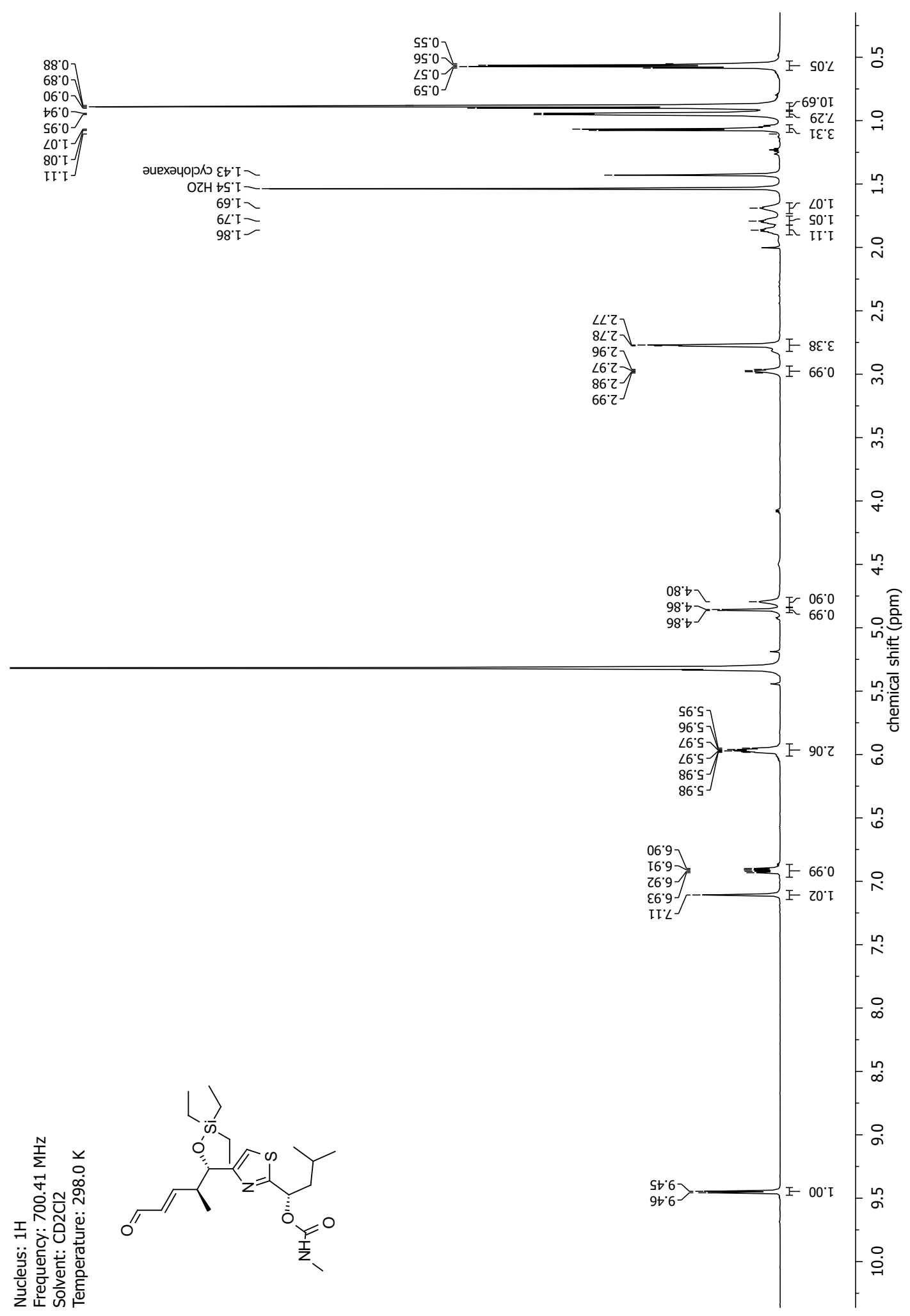




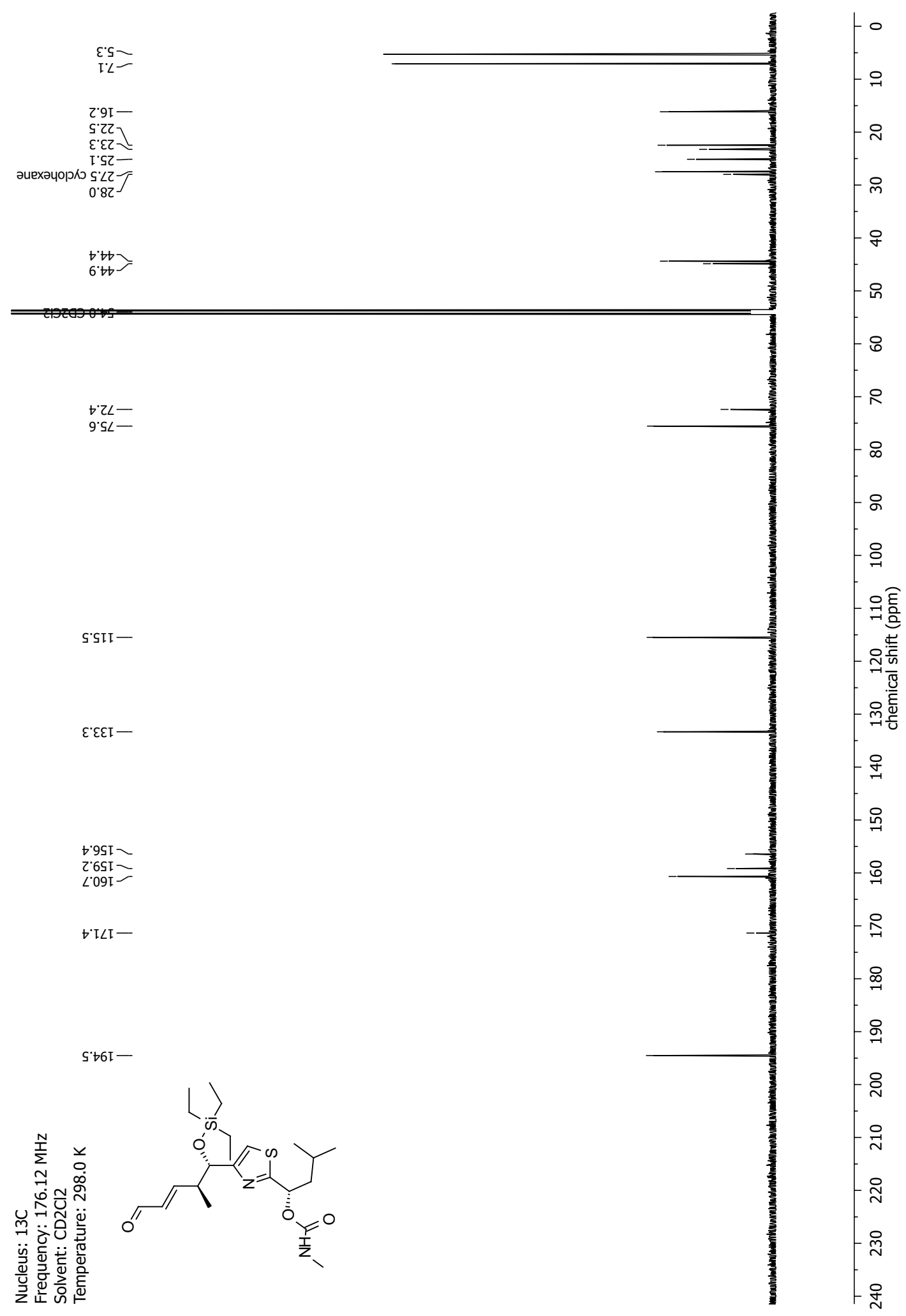


NMR-Spectra for Compound 12

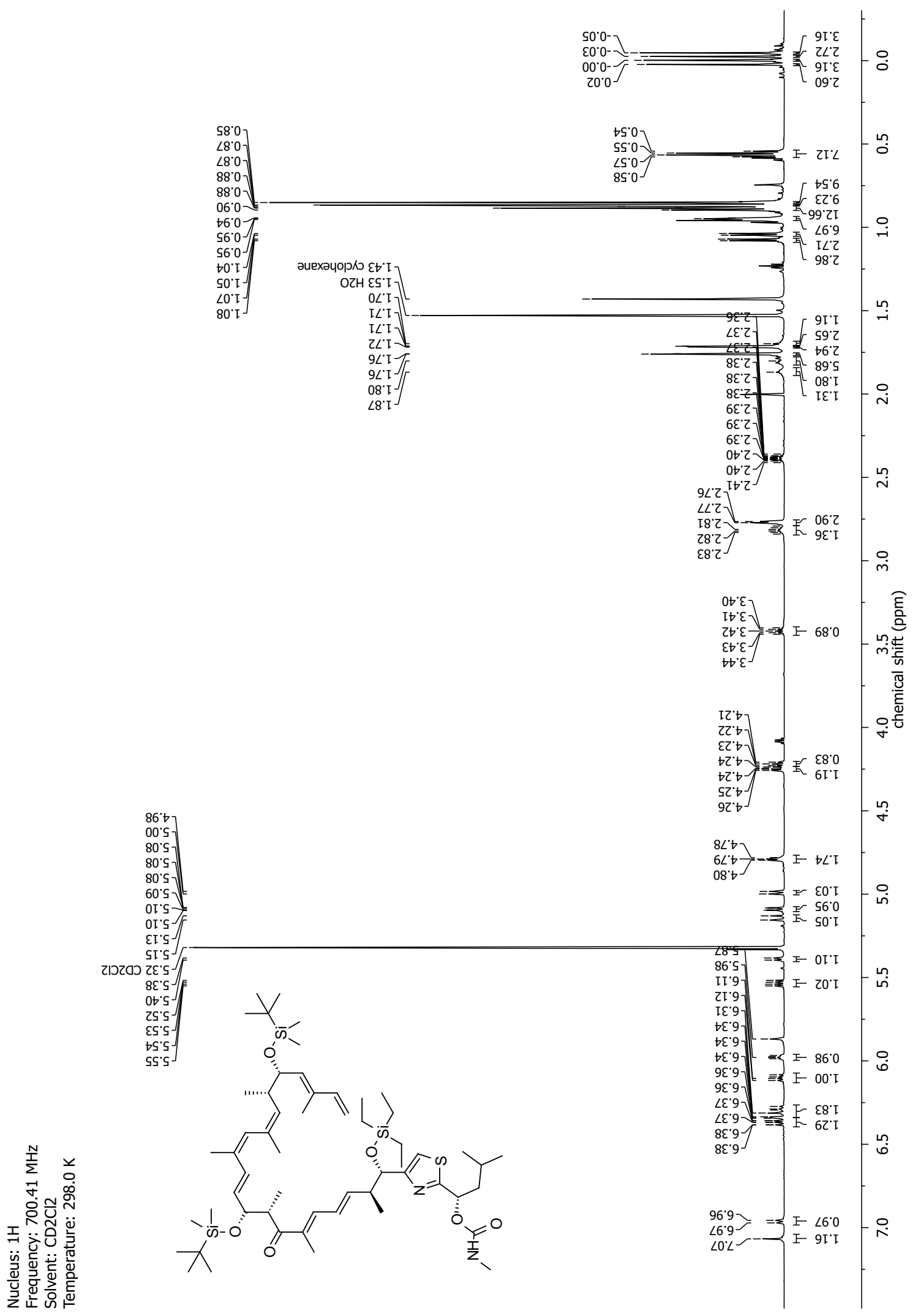




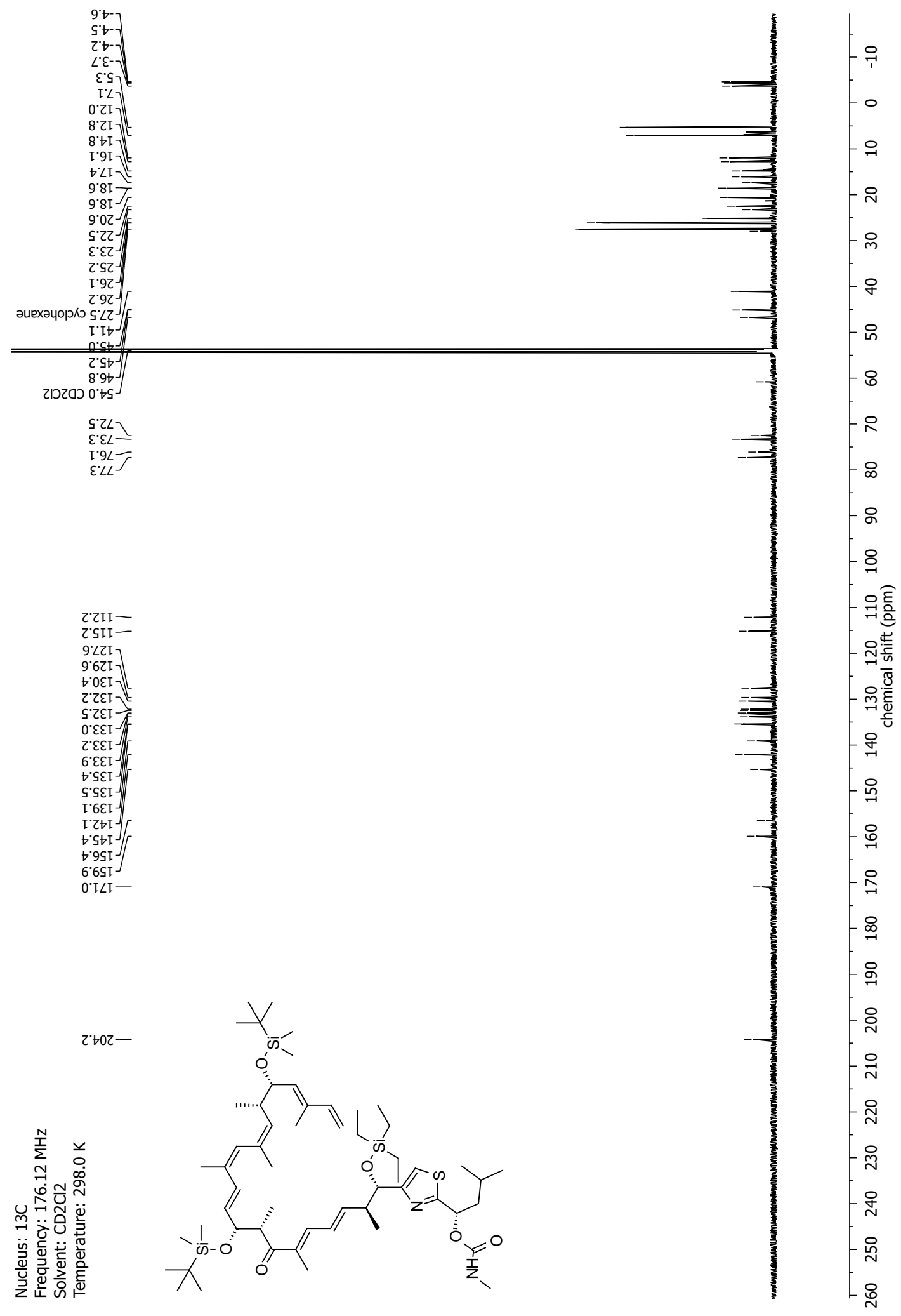


NMR-Spectra for Compound 13

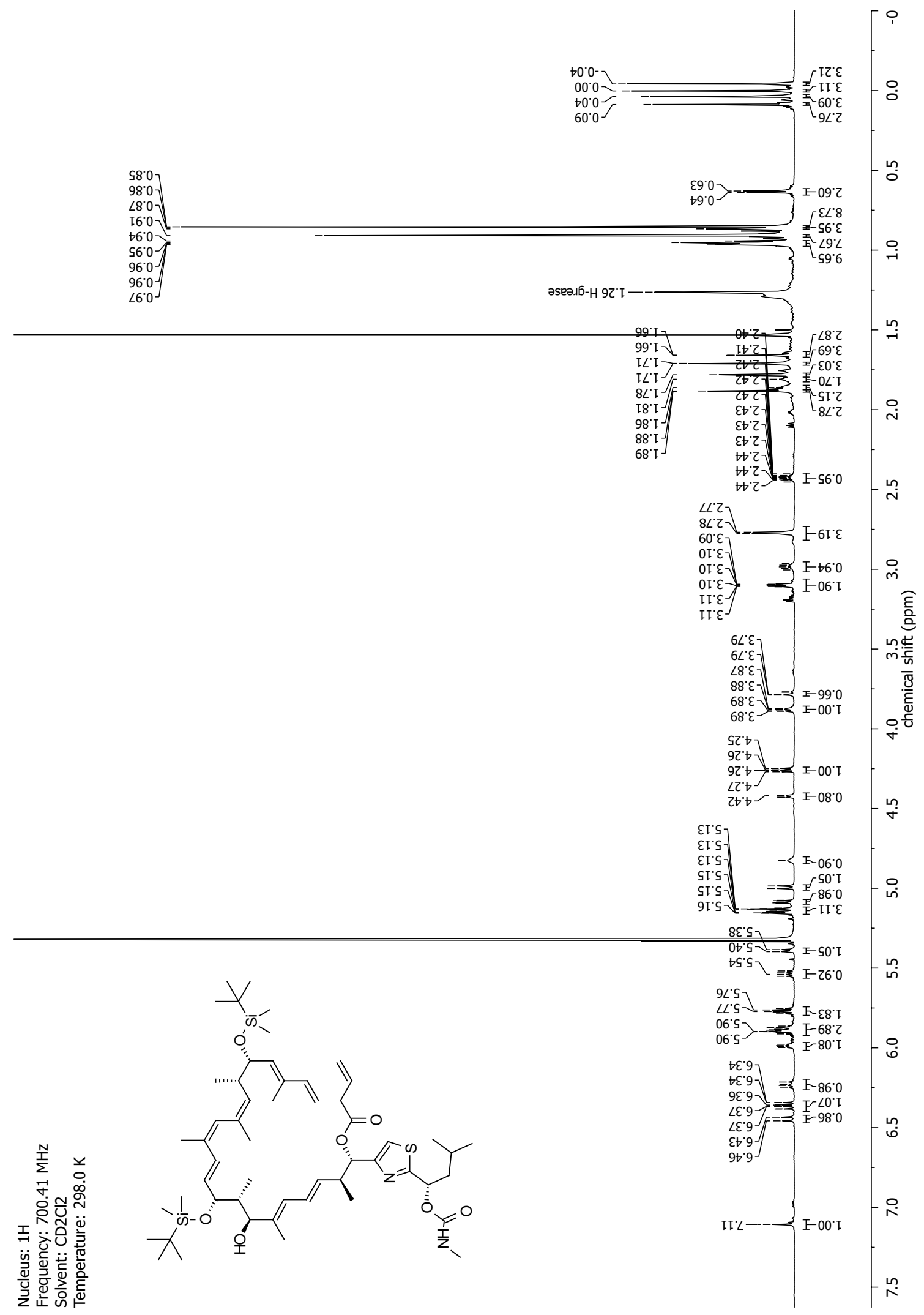




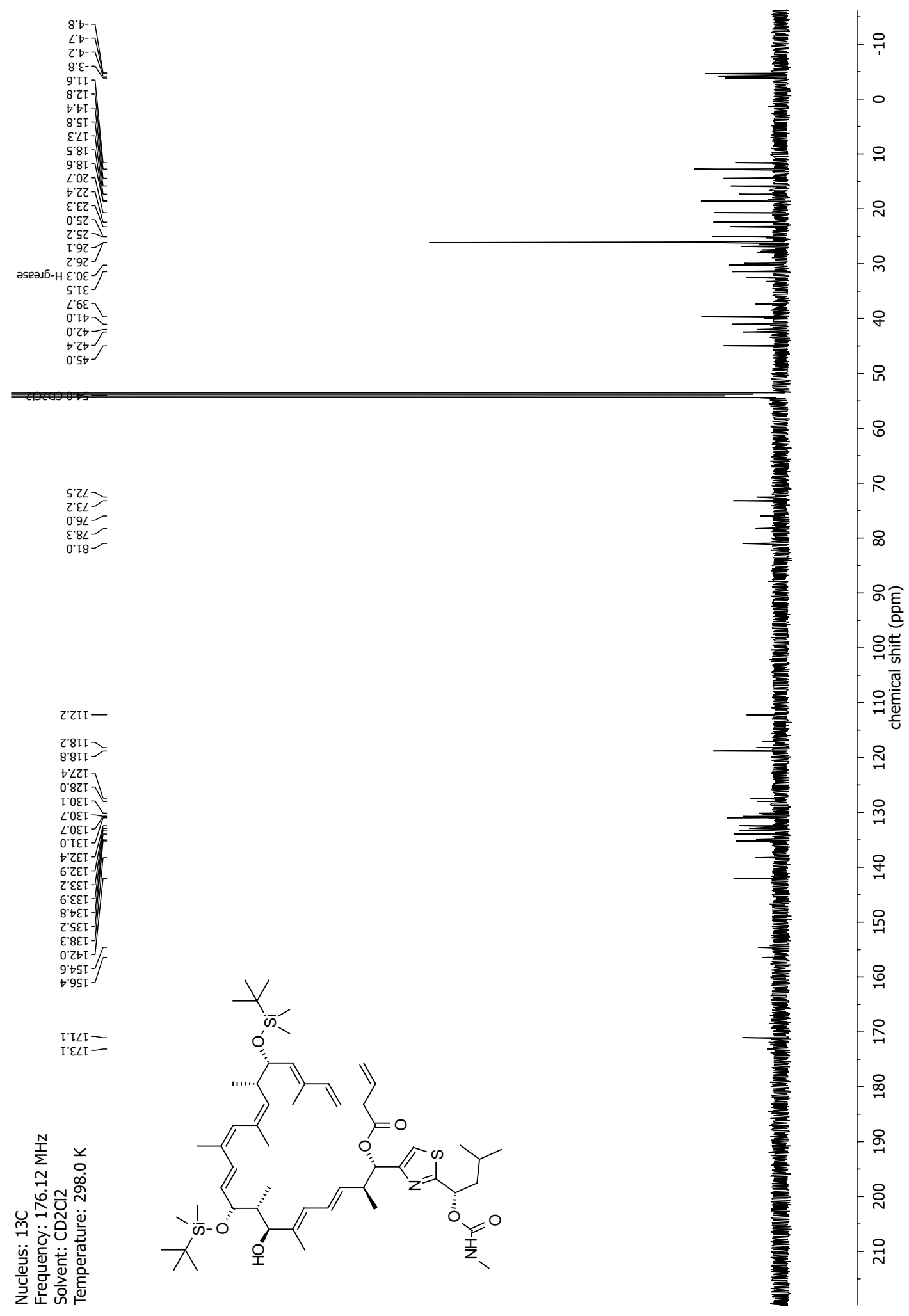




\section{NMR-Spectra for Compound 6}

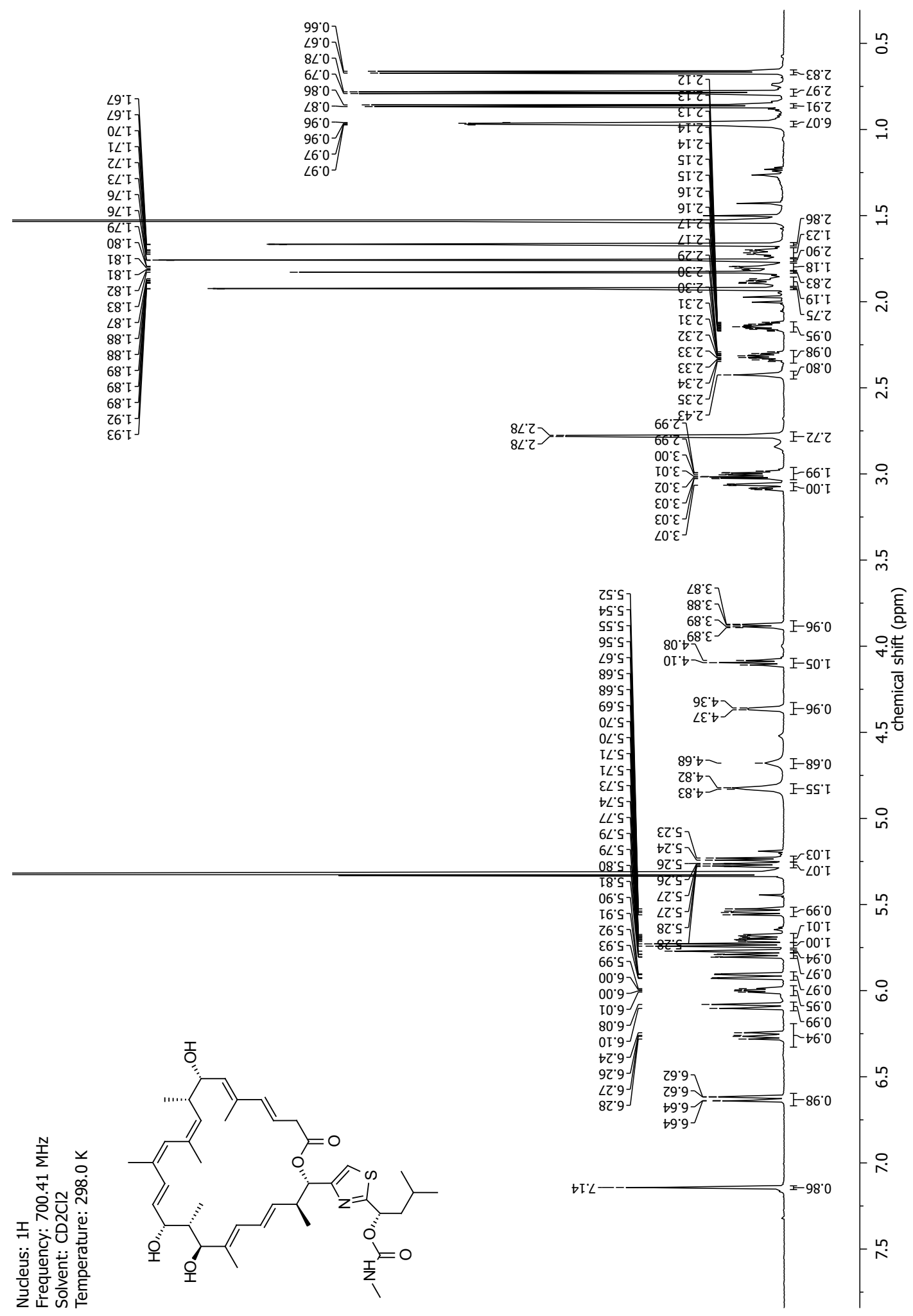



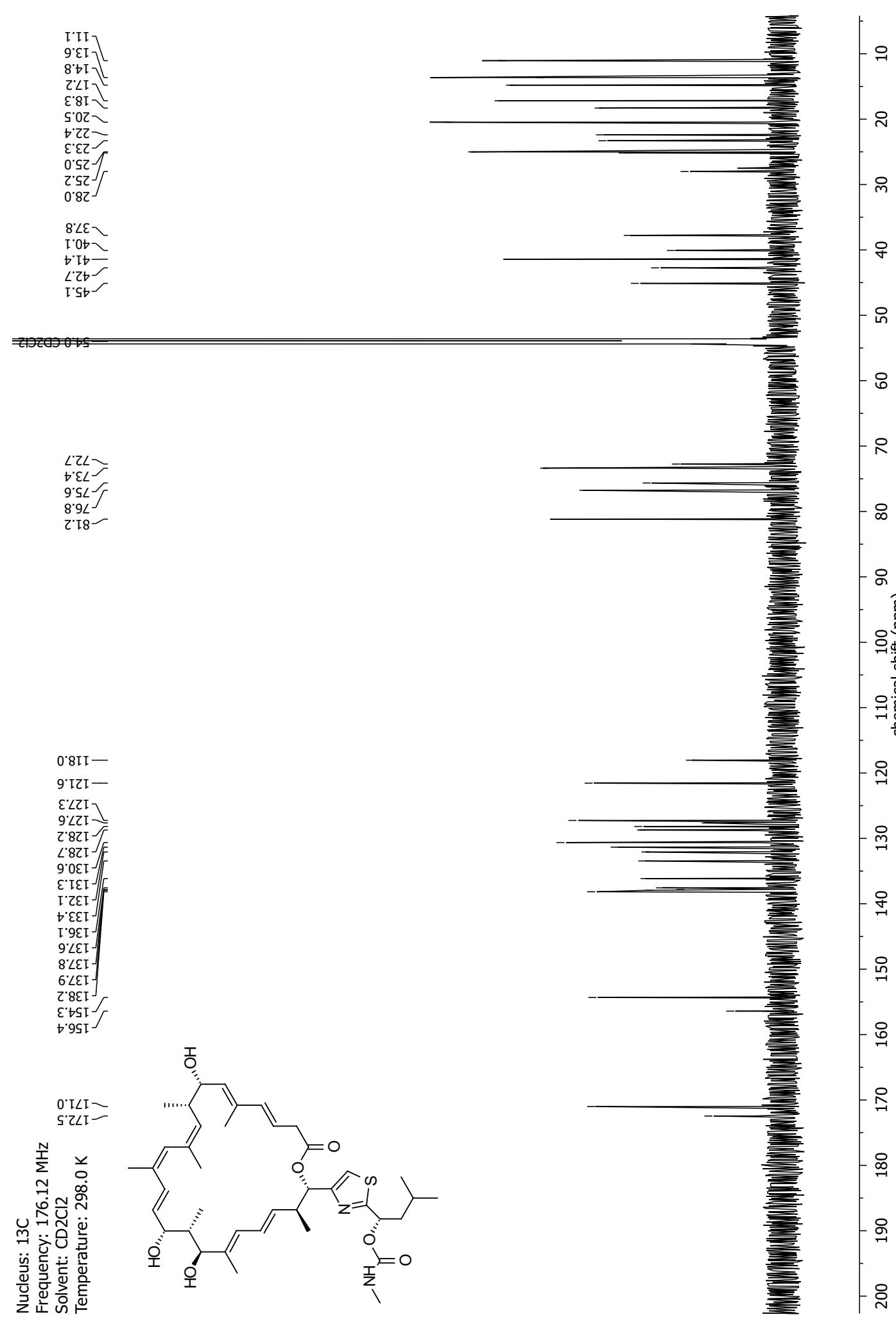

으

8

ㅇ

$\infty$

8

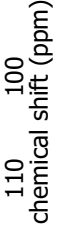

్ㅗ

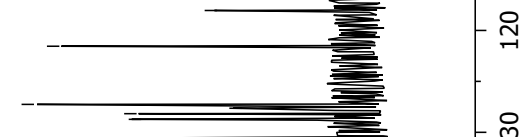

$\stackrel{m}{9}$

g

옥

$\stackrel{\circ}{\circ}$

옥

$\stackrel{\infty}{\sim}$

욤

우 


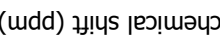

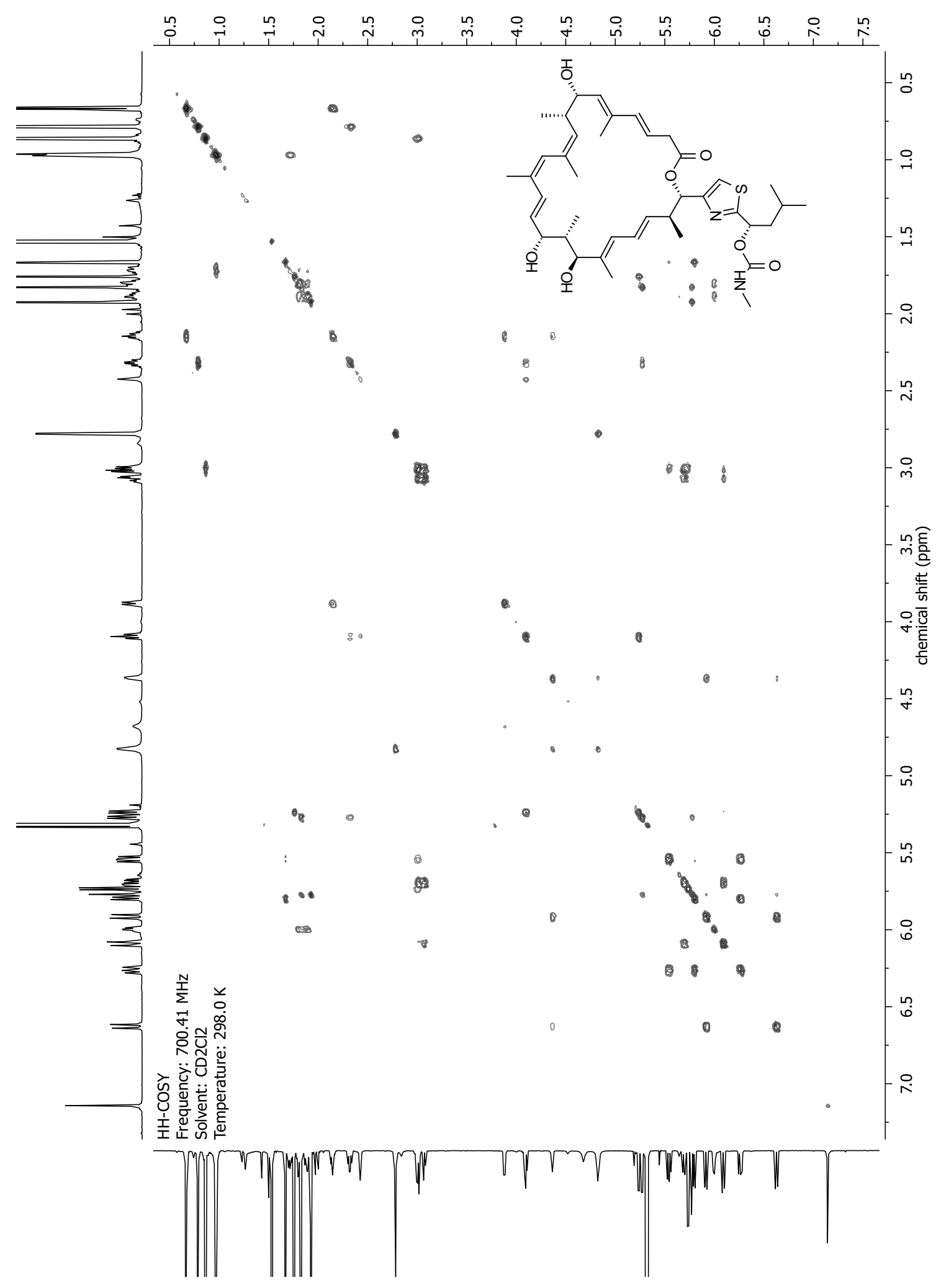


(mdd) भ!บऽ ןеग!шәцว

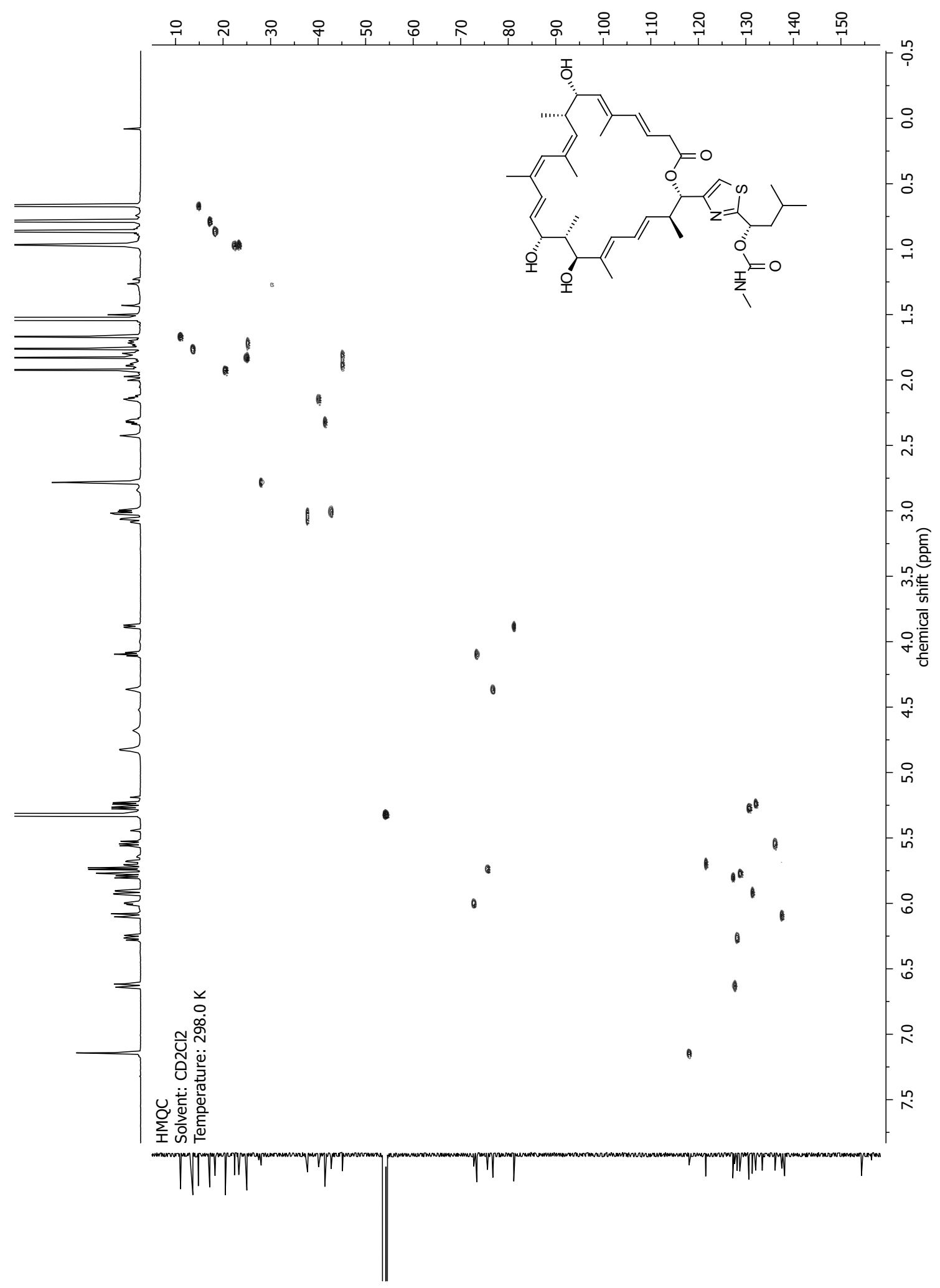


(mdd) भ!บ५ ןอว!ฒәцว

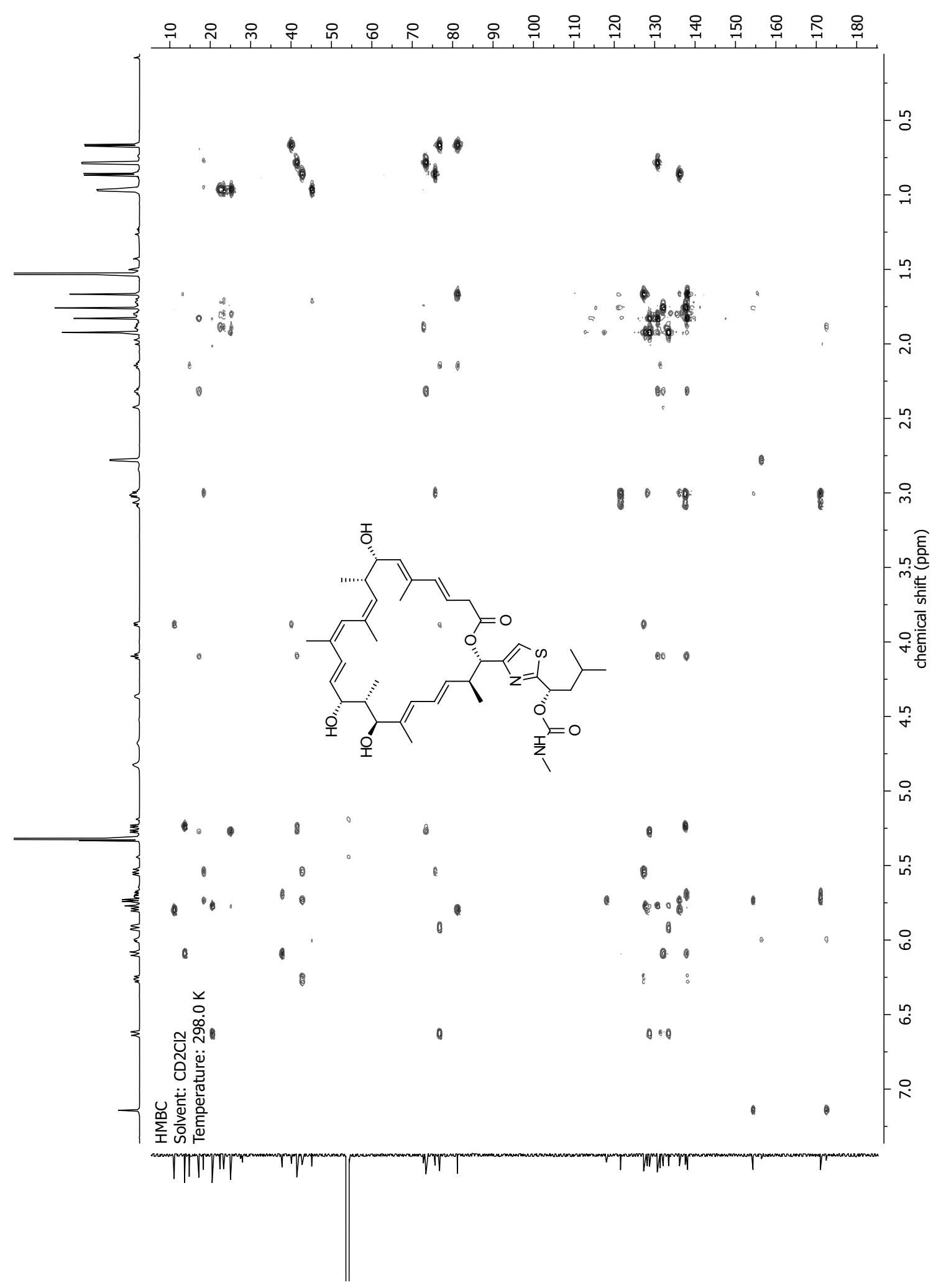


NMR-Spectra for Compound 17

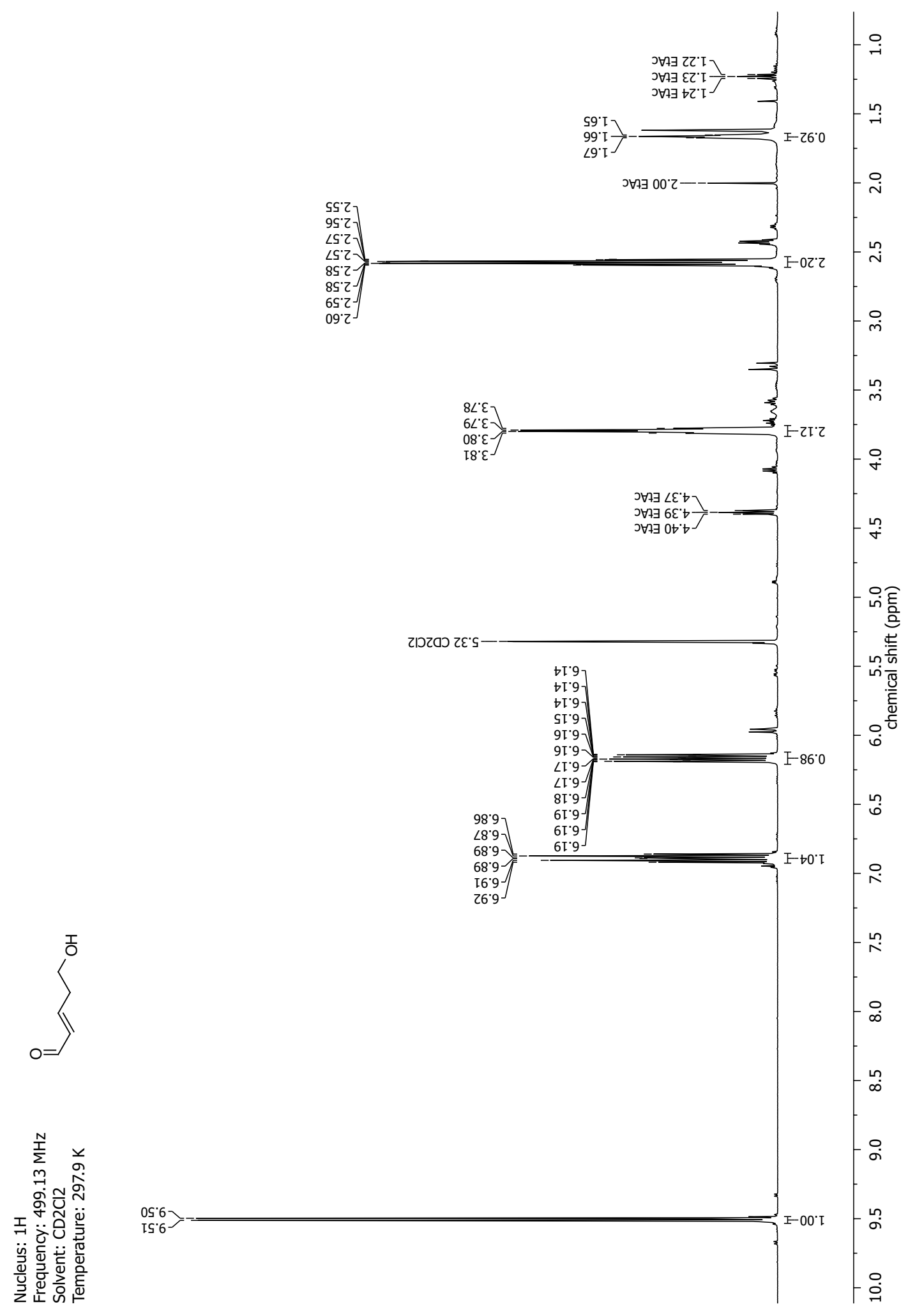



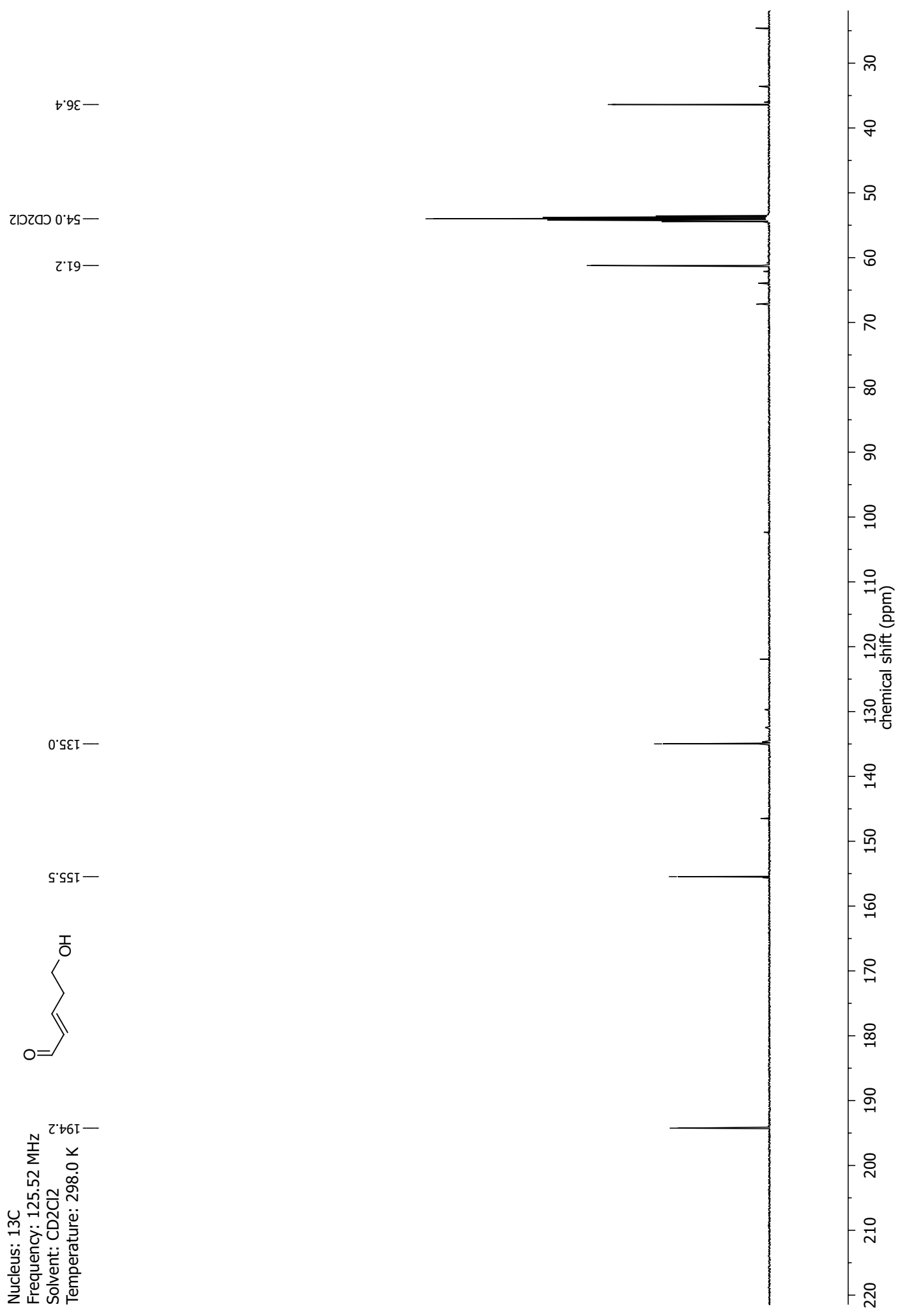
NMR-Spectra for Compound 15

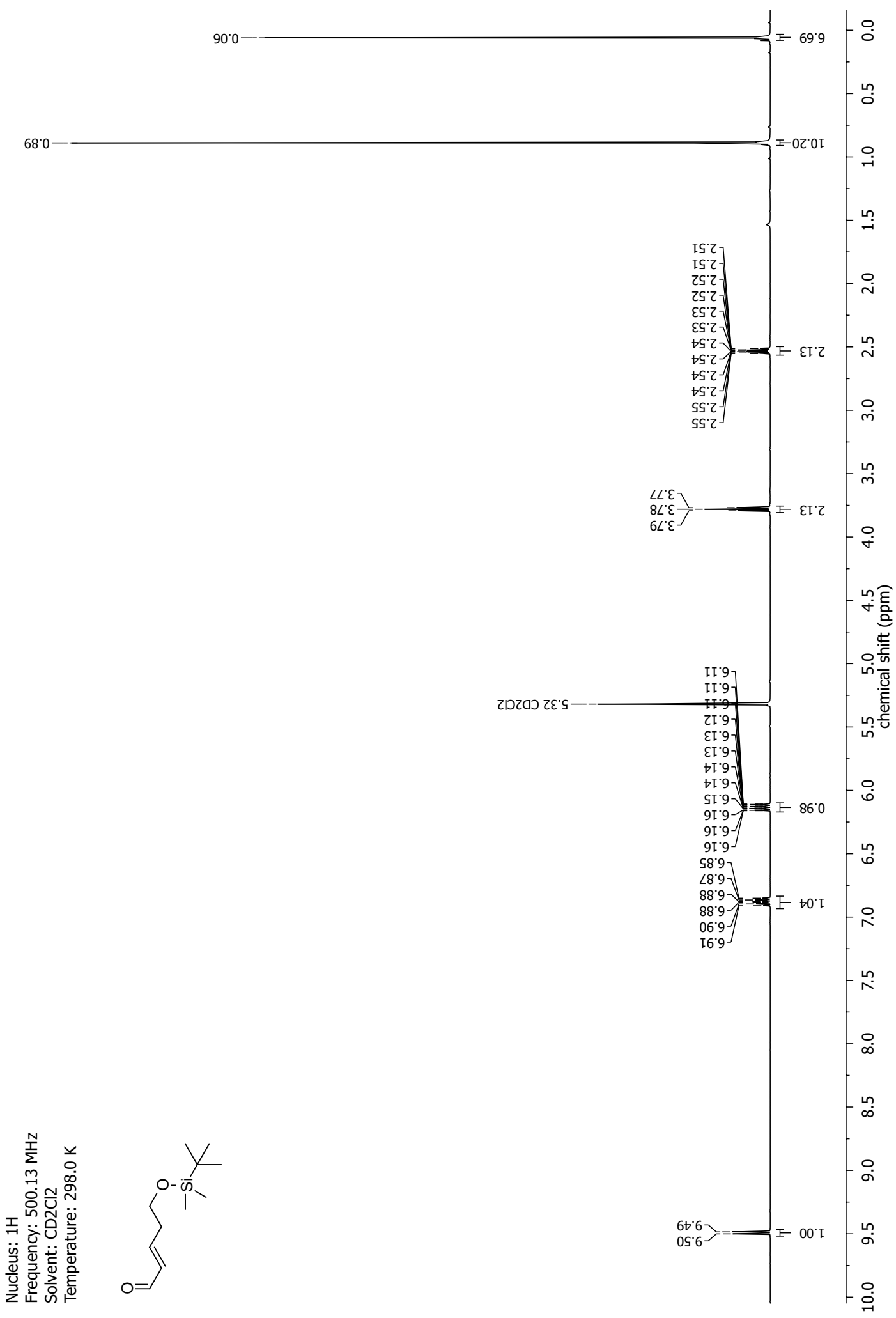



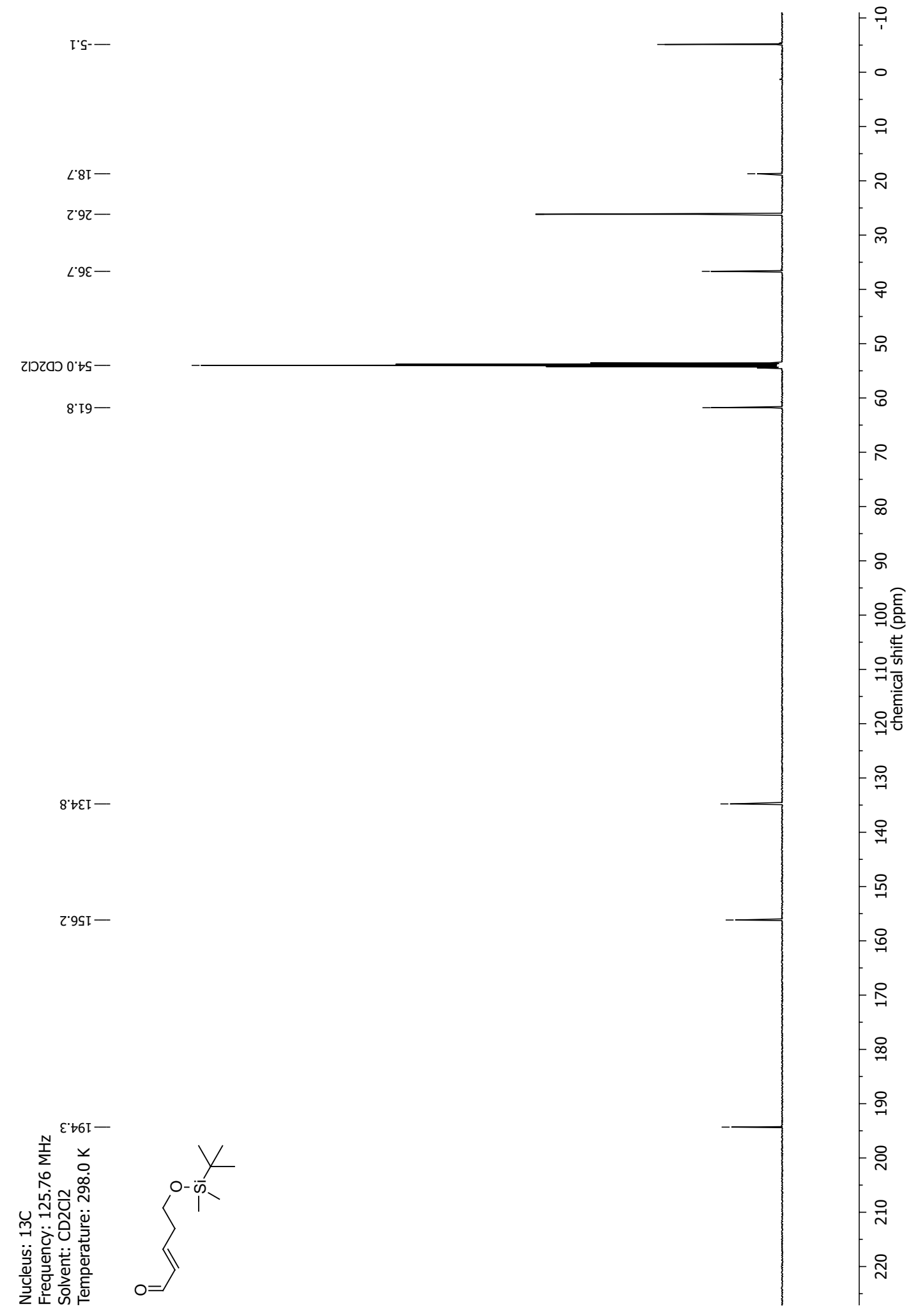
NMR-Spectra for Compound 18

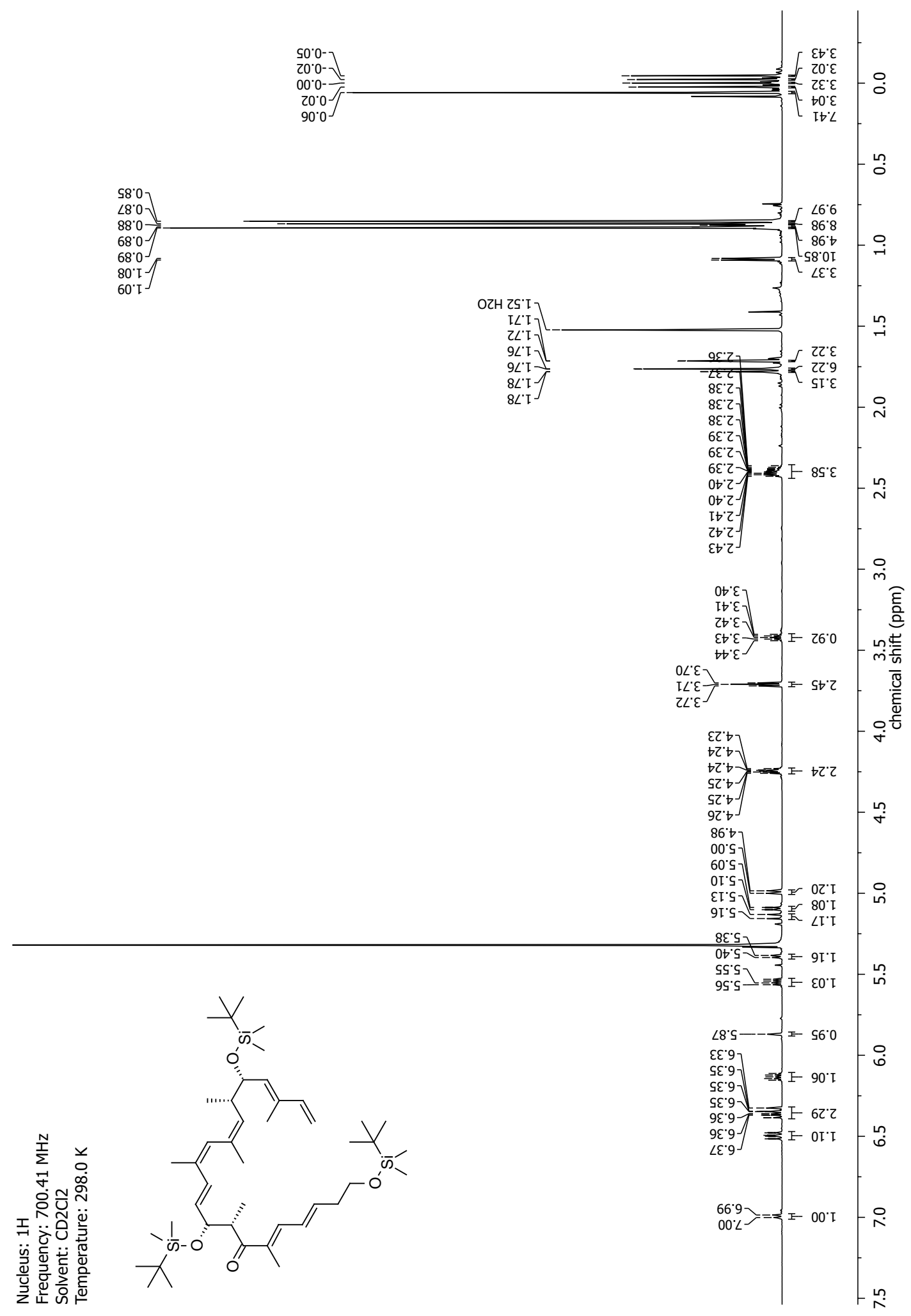




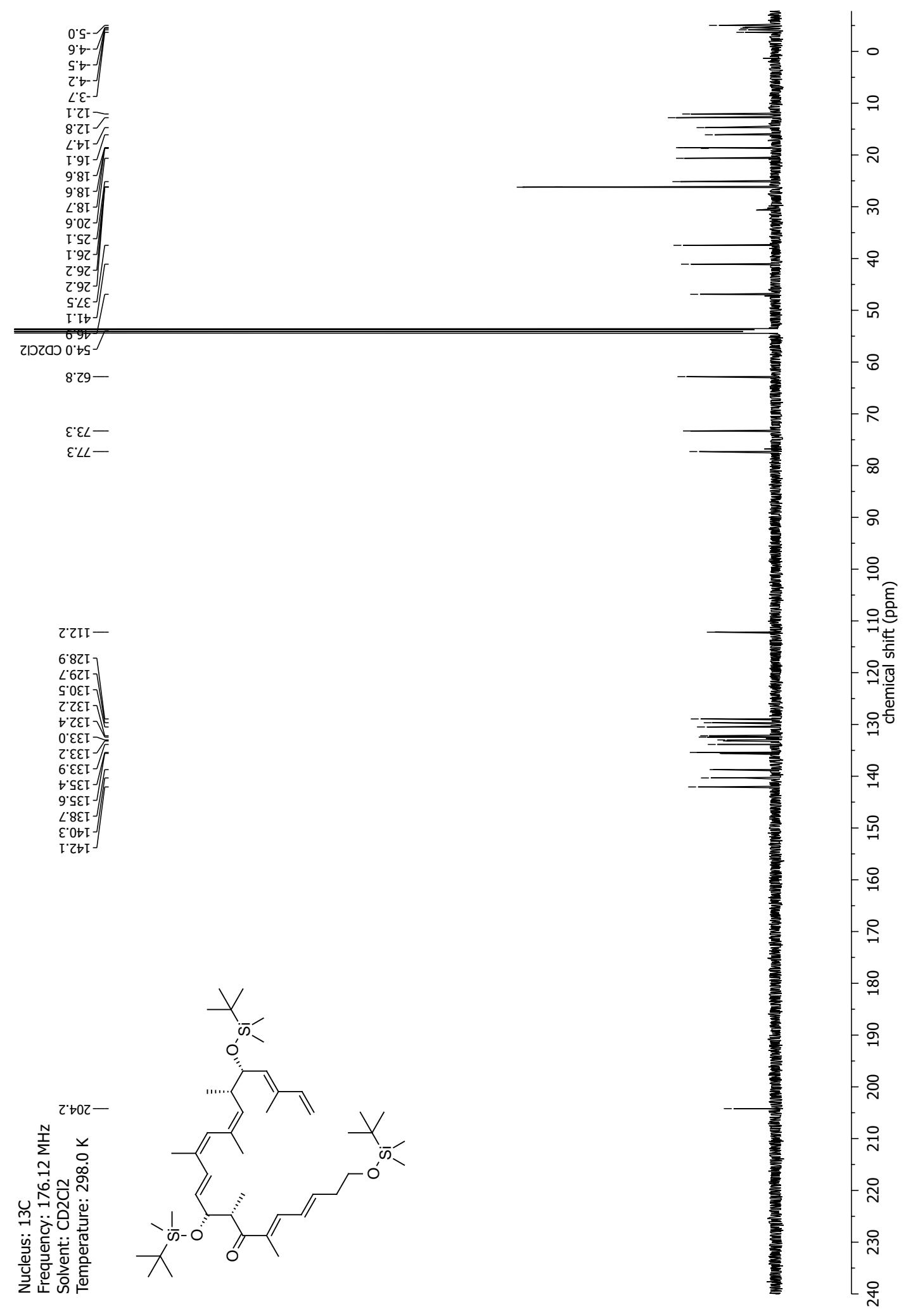


NMR-Spectra for Compound 22

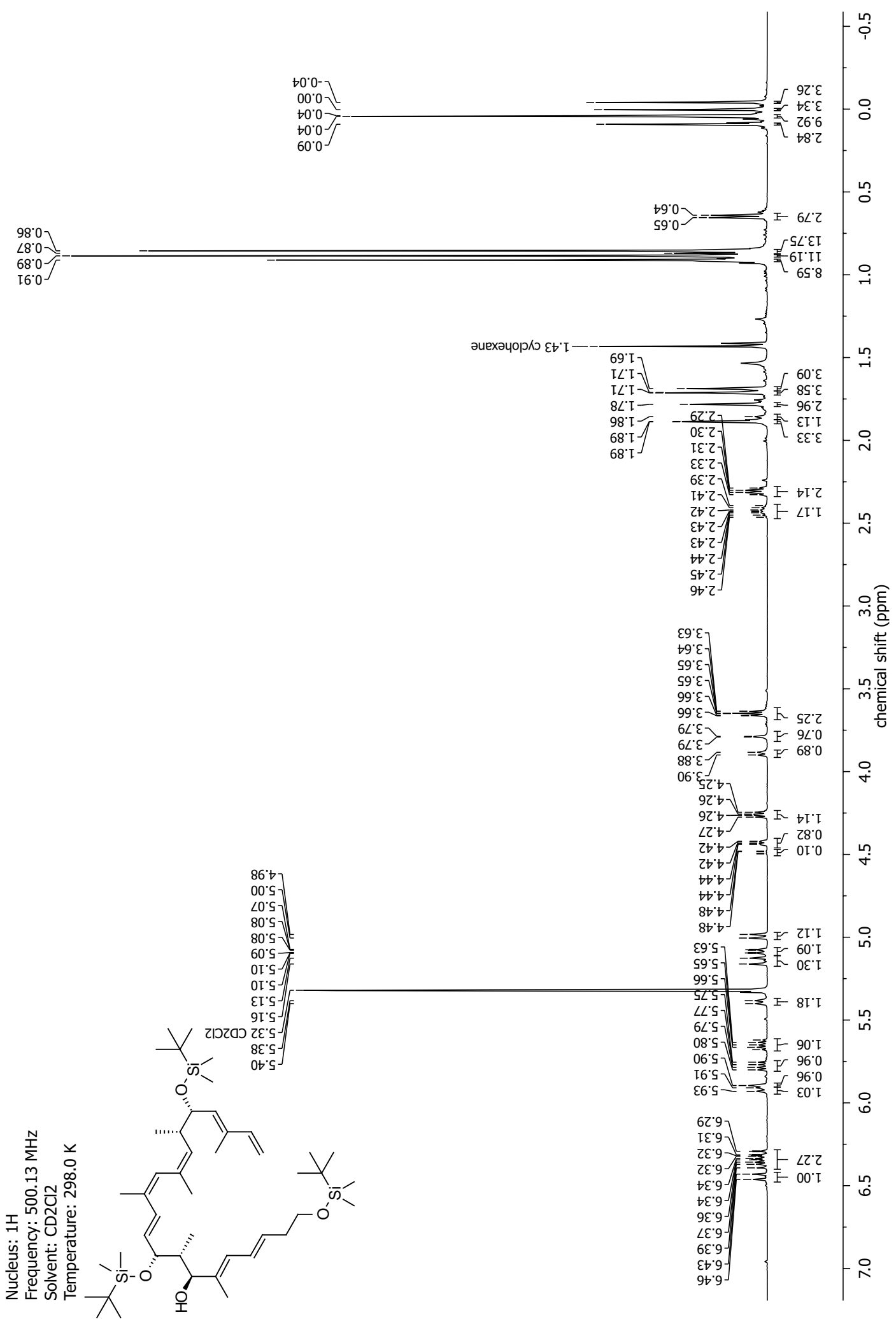




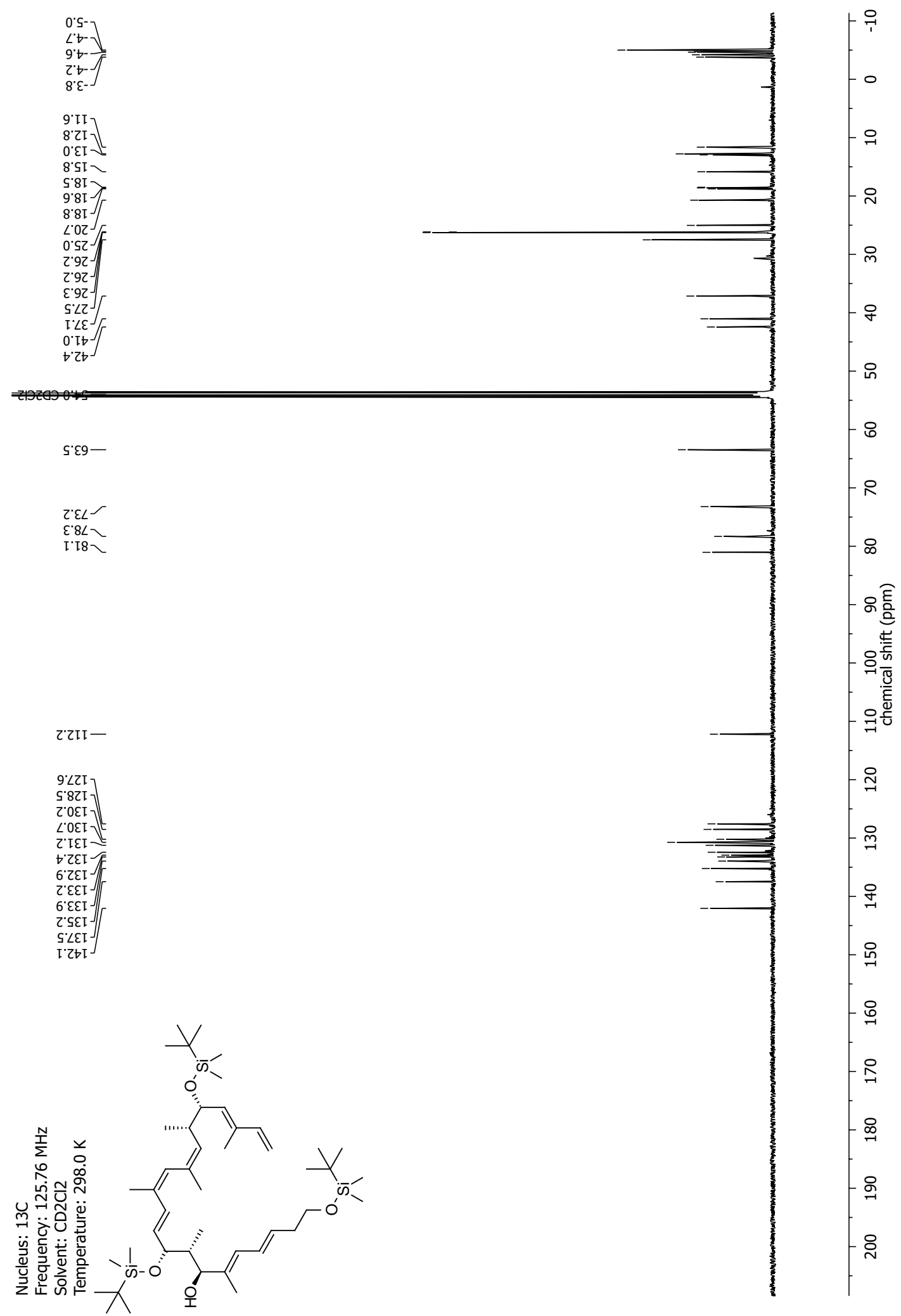


NMR-Spectra for Compound 19

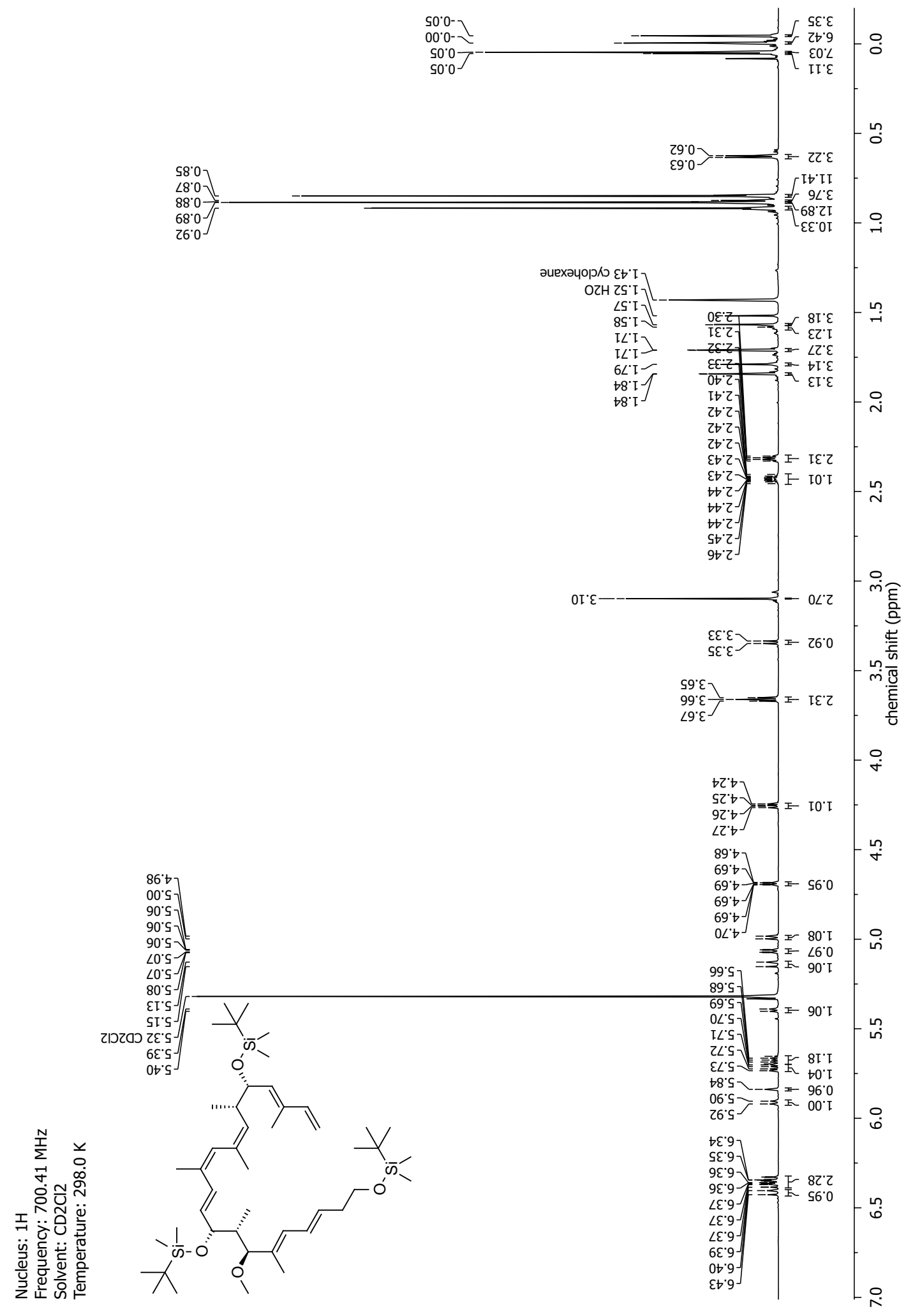




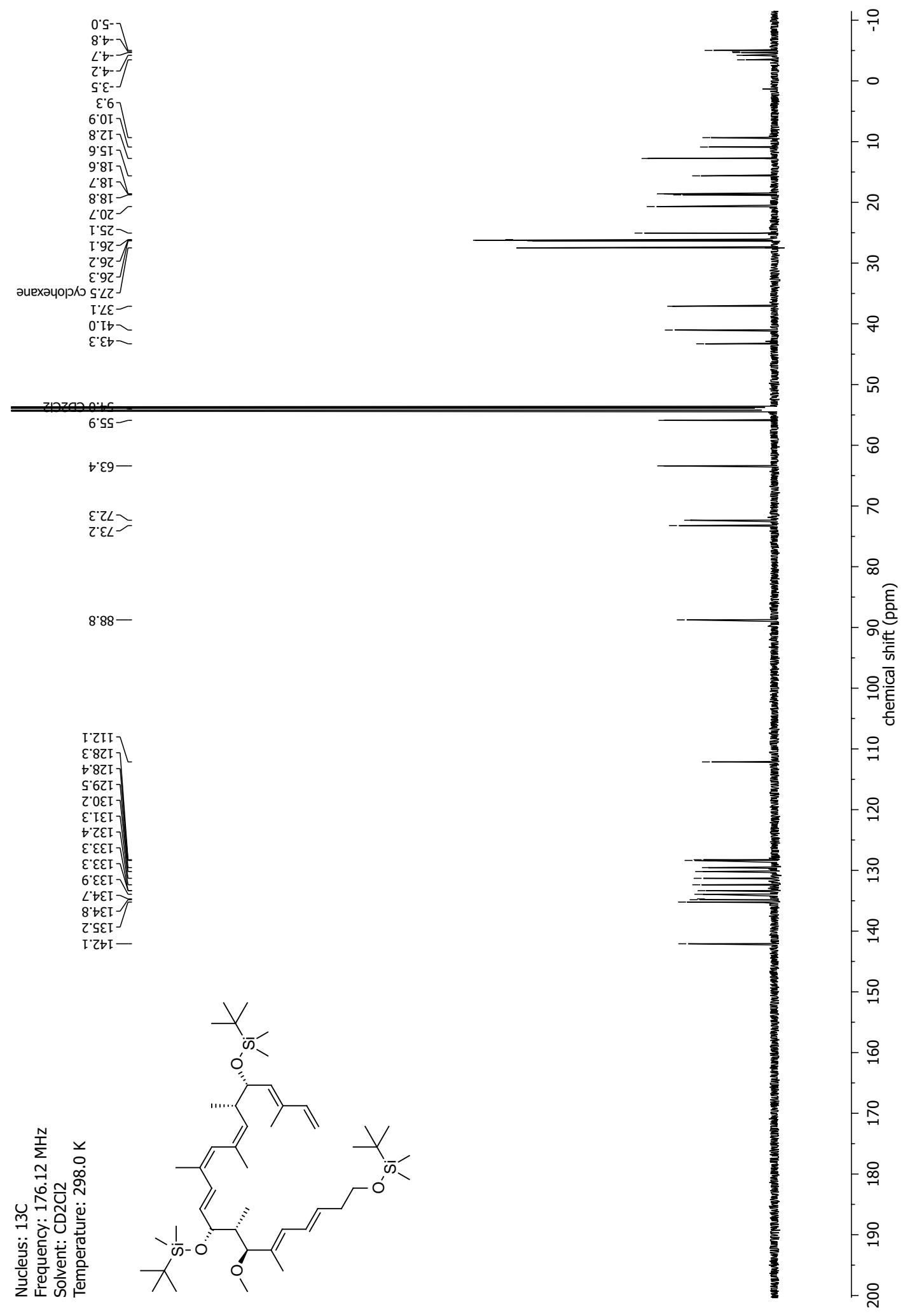


NMR-Spectra for Compound 23






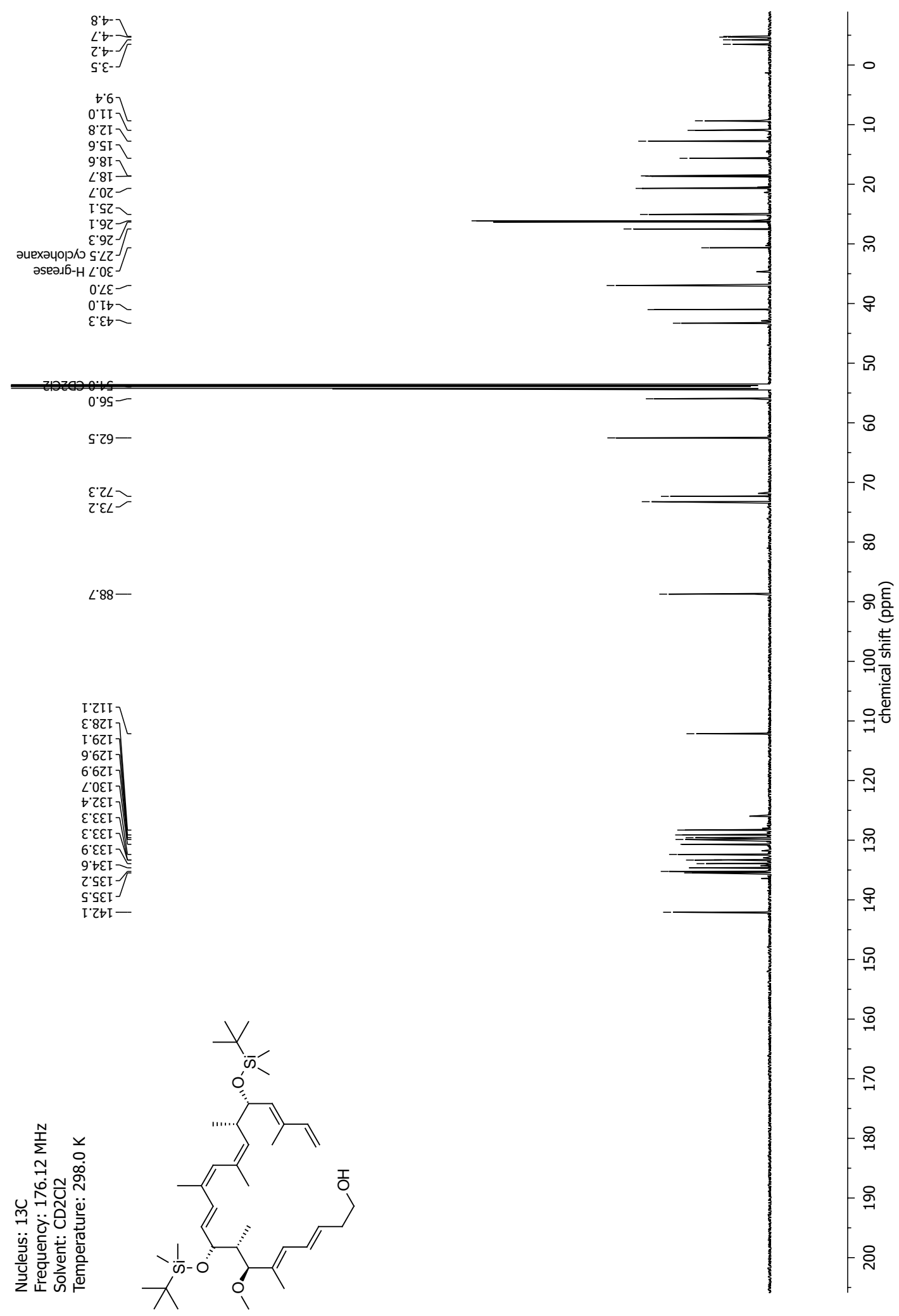


NMR-Spectra for Compound 20





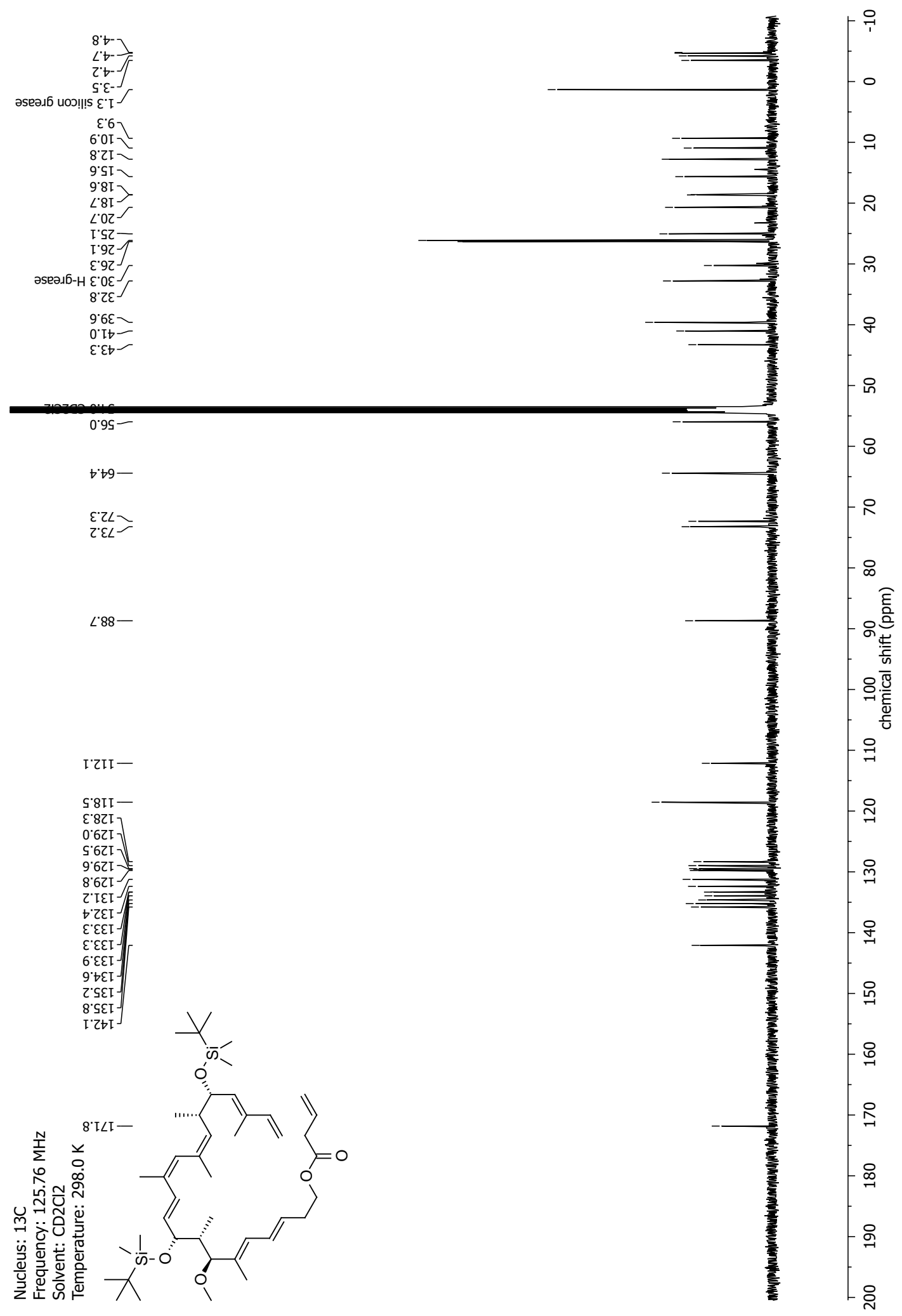
NMR-Spectra for Compound 7

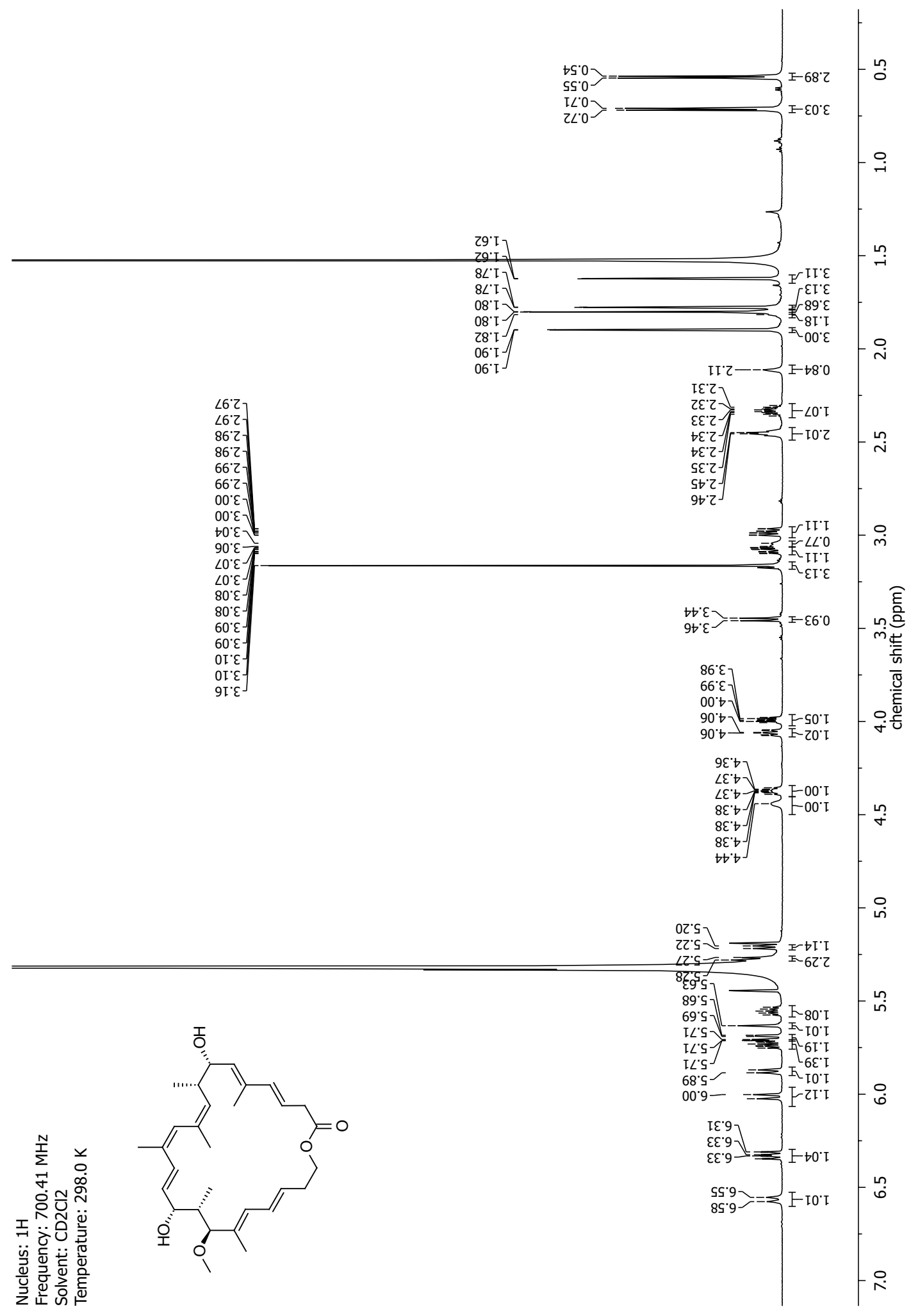







(udd) भ!บऽ ןอว!ฒәцว

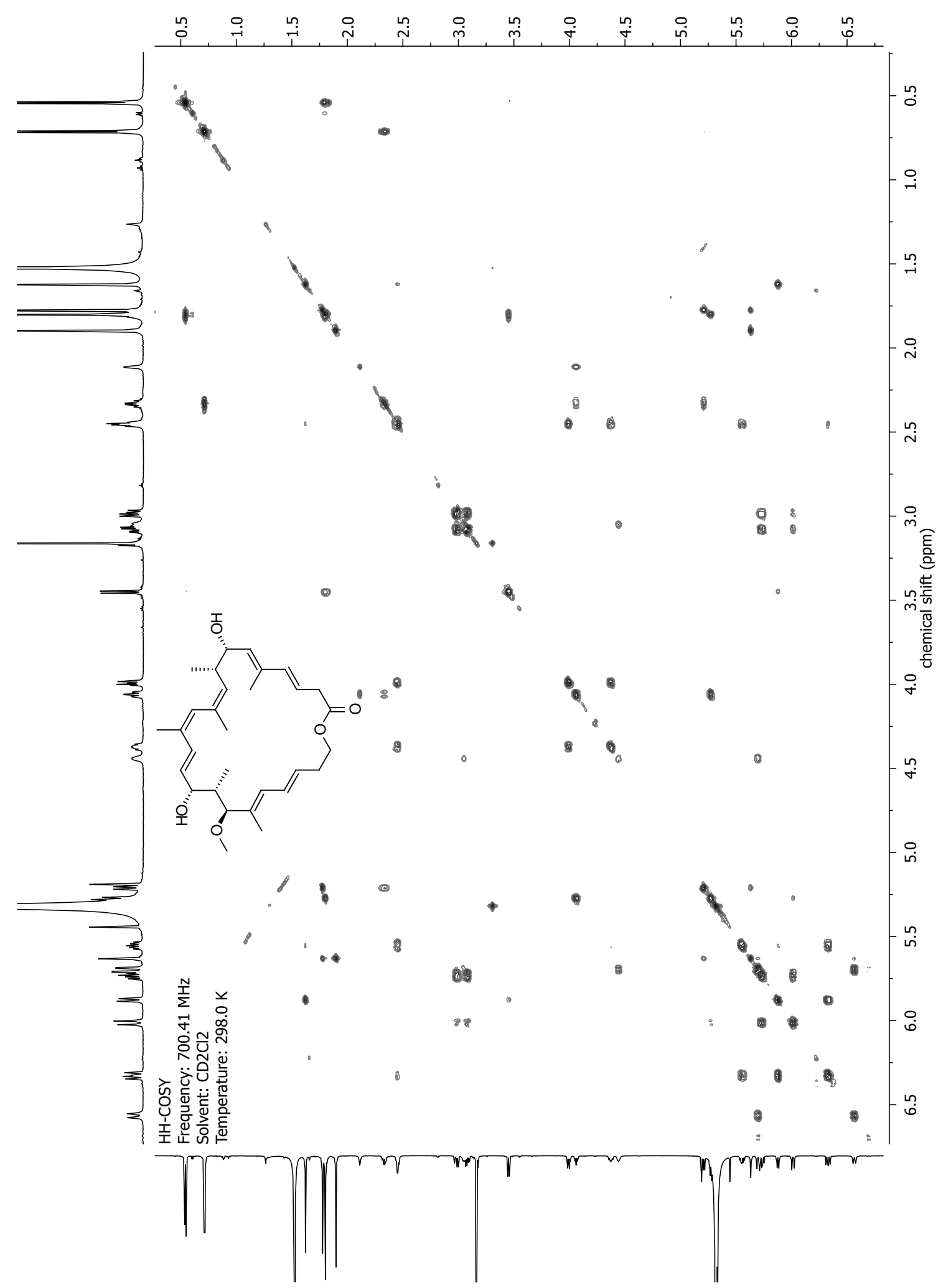


(udd) भ!บऽ ןอว!ฒәцว

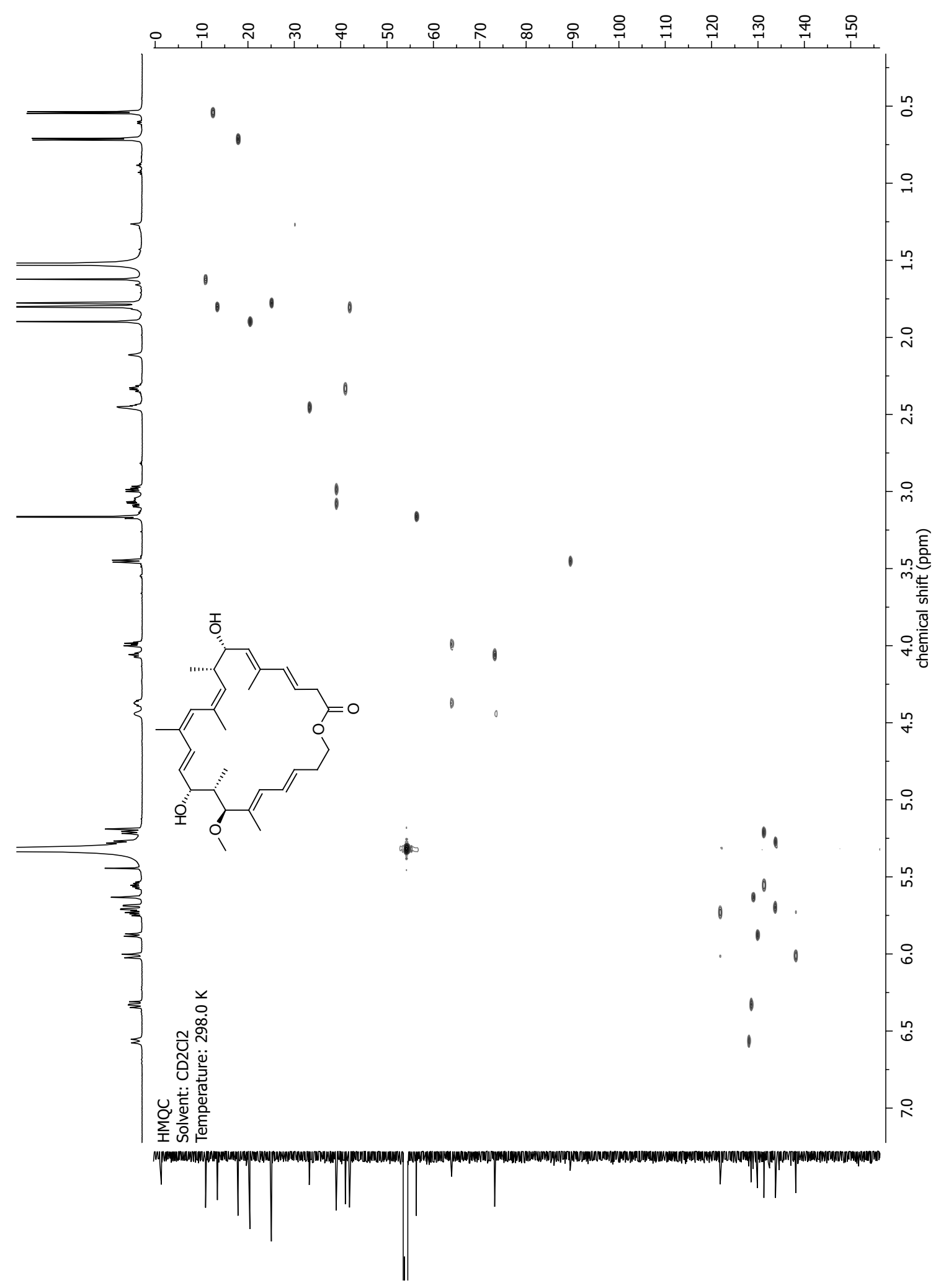


(udd) भ!บऽ ןอว!ฒәцว

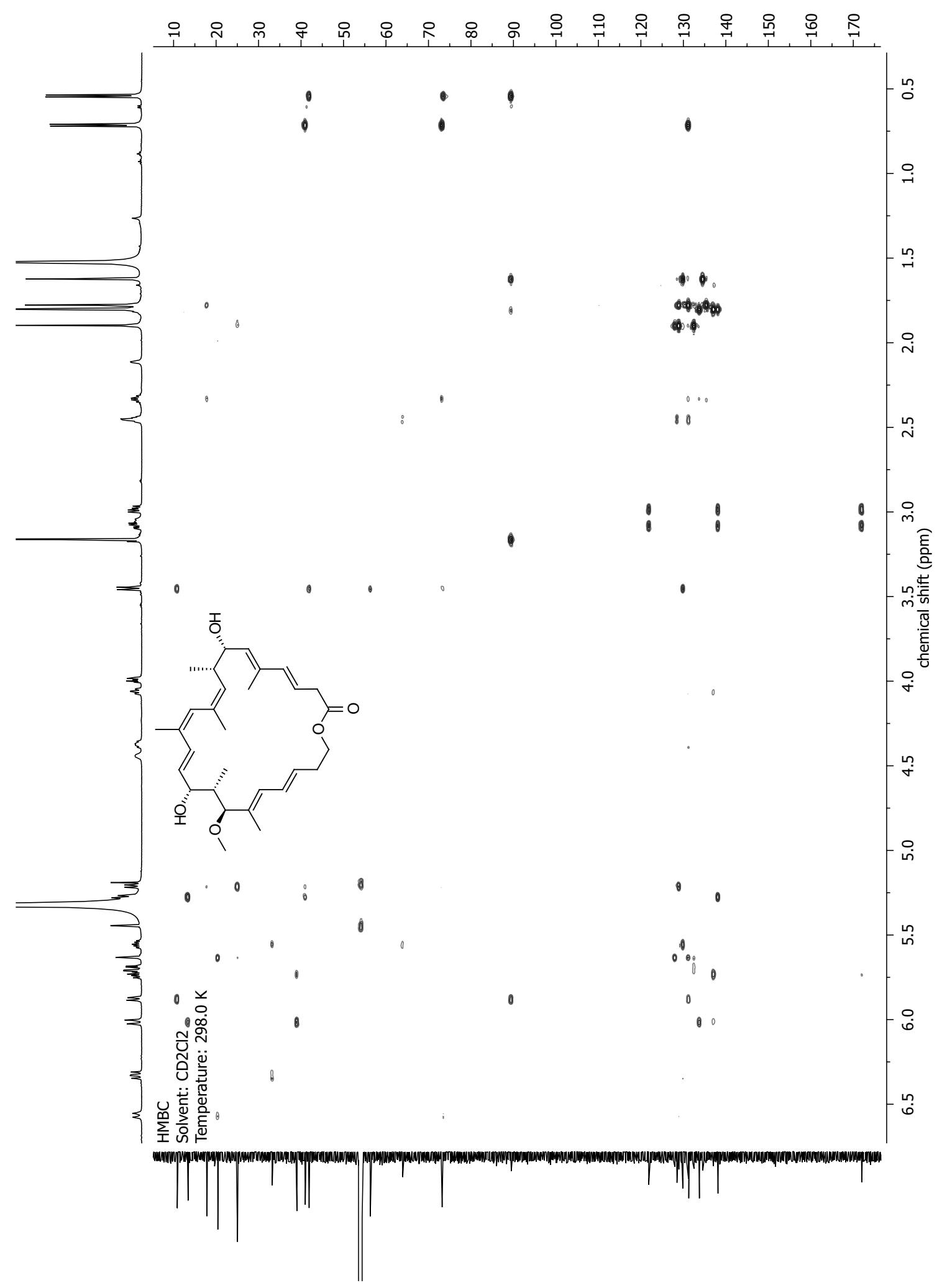

